\title{
WestVirginiaUniversity
}

THE RESEARCH REPOSITORY @ WVU

Graduate Theses, Dissertations, and Problem Reports

2000

\section{Effect of debris-induced lift-off on magnetic flux leakage inspection results}

Francisco Louis Valentine
West Virginia University

Follow this and additional works at: https://researchrepository.wvu.edu/etd

\section{Recommended Citation}

Valentine, Francisco Louis, "Effect of debris-induced lift-off on magnetic flux leakage inspection results" (2000). Graduate Theses, Dissertations, and Problem Reports. 1035.

https://researchrepository.wvu.edu/etd/1035

This Thesis is protected by copyright and/or related rights. It has been brought to you by the The Research Repository @ WVU with permission from the rights-holder(s). You are free to use this Thesis in any way that is permitted by the copyright and related rights legislation that applies to your use. For other uses you must obtain permission from the rights-holder(s) directly, unless additional rights are indicated by a Creative Commons license in the record and/ or on the work itself. This Thesis has been accepted for inclusion in WVU Graduate Theses, Dissertations, and Problem Reports collection by an authorized administrator of The Research Repository @ WVU. For more information, please contact researchrepository@mail.wvu.edu. 
Effect of Debris-Induced Lift-off on Magnetic Flux Leakage Inspection Results

Francisco Valentine, P.E.

\author{
Thesis submitted to the \\ College of Engineering and Mineral Resources \\ at West Virginia University \\ in partial fulfillment of the requirements \\ for the degree of
}

Master of Science

In

Mechanical Engineering

Kenneth Means, Ph.D., Chair

Alfred Stiller, Ph.D.

Nigel Clark, Ph.D.

Department of Mechanical and Aerospace Engineering

Morgantown, West Virginia

2000

Key words: Magnetic Flux Leakage, Nondestructive Testing, Lift-off, Debris, Pipe Inspection 


\section{ABSTRACT \\ Effect of Debris-Induced Lift-off on Magnetic Flux Leakage Inspection Results \\ By Francisco Valentine, P.E.}

Pipeline cleanliness is often taken for granted by pipeline operators. Debris is present in all pipelines and has the potential to affect the acquired magnetic flux leakage data. In sufficient amounts, debris causes lift-off of the sensors and/or the magnetizer of the magnetic flux leakage inspection tool.

A test apparatus was developed to qualitatively examine the effect of debris-induced lift-off on magnetic flux leakage data. The results of this research indicate that debrisinduced lift-off has a detrimental effect which increases the potential of pipeline defects being either undersized or missed. It has long been thought that small amounts of debris do not affect magnetic flux leakage data. However, results of this testing indicate that even small amounts of debris-induced lift-off can significantly reduce the magnitude of the acquired peak magnetic flux leakage signal. This may ultimately compromise the safety of the pipeline and the surrounding population.

To reduce or eliminate the potential for underestimating or missing injurious pipeline defects, the pipeline should be regularly cleaned. This minimizes the occurrence of large accumulations of debris. Prior to a magnetic flux leakage inspection, the line should also be evaluated to determine whether debris is present. If it determined that debris is present, even in small quantities, the pipeline should be cleaned using the appropriate pipeline cleaning method. This will improve the quality of the magnetic flux leakage inspection data and resulting integrity assessment. 


\section{ACKNOWLEDGMENTS}

I would like to take this opportunity to express my gratitude to those people who have made a positive impact in my life. These include: my son, Louis X. Valentine, Caridad and Bob Pecore, my father, Louis B. Valentine, Jimmy and Kay Reese, Steve Wilkerson, Alvin Walker, Eric Stevens, John Tiratsoo, and Maria Watson. A special thanks is extended to Mr. and Mrs. Burdette whose concern, interest, and love has helped me to realize that I can accomplish anything I set my mind on.

I would like to thank Gwen Walker (Columbia Gas Transmission Corporation) for providing me with an opportunity to prove myself while at Sedalia, WV. I would also like to extend appreciation to Bill Butterworth (Columbia Gas Transmission Corporation) for giving me an opportunity to prove myself at Strasburg, VA, inspiring me to acquire Professional Engineering licensure, and for allowing me to perform the testing upon which this thesis is based.

I thank my committee: Kenneth Means, Nigel Clark, and Alfred Stiller for your time and consideration.

I am also appreciative of the industry reviewers who have provided technical comments throughout the development of this thesis:

Pat Porter

Bruce Nestleroth

Rick Desaulniers

Jonathan Luedke
Clockspring Company

Battelle Memorial Institute

Pipeline Integrity International

Columbia Gas Transmission Corporation 


\section{TABLE OF CONTENTS}

BSTRACT

ii

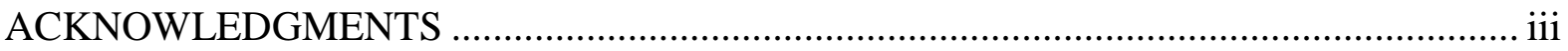

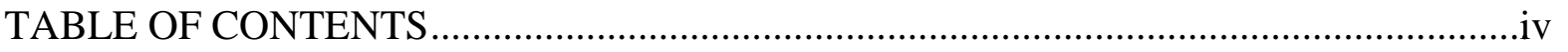

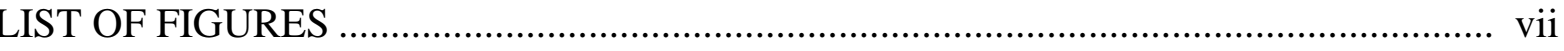

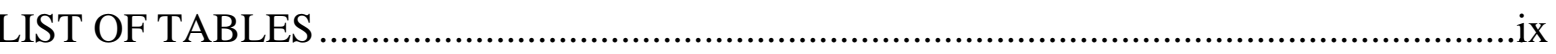

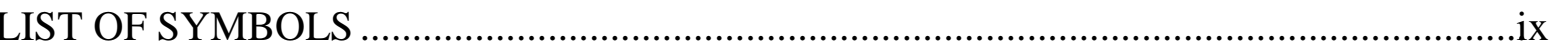

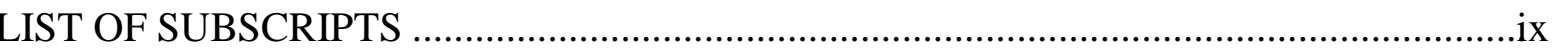

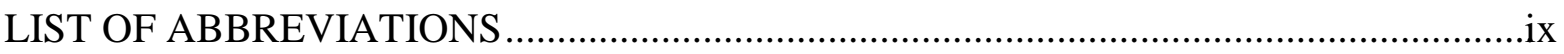

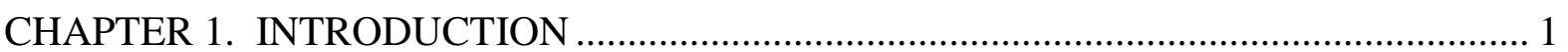

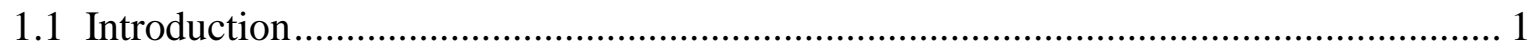

1.2 Problem Statement .................................................................................................. 1

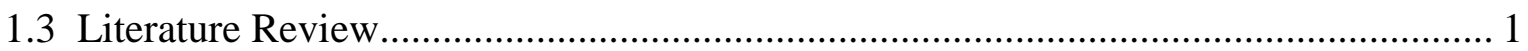

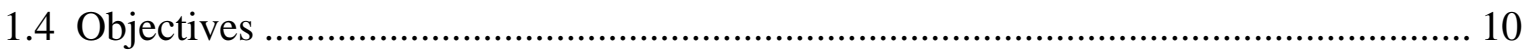

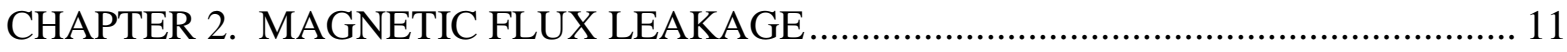

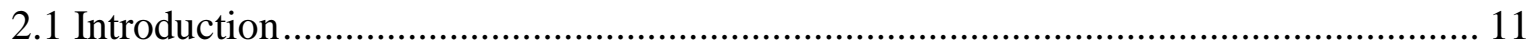

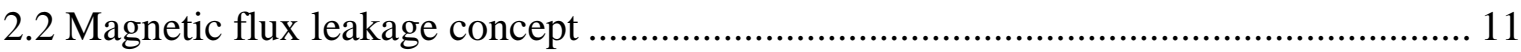

2.3 Components of an magnetic flux leakage inspection tool ................................ 13

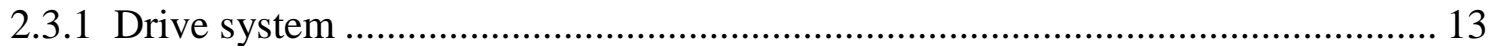

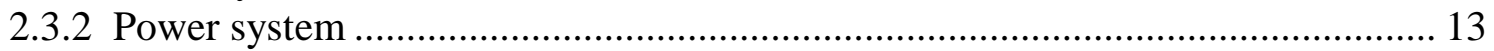

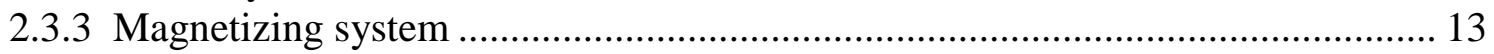

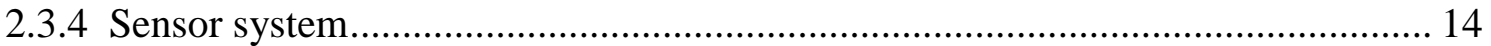

2.3 .5 Data conditioning, acquisition, and storage system .................................... 15

2.4 Pipeline industry assumptions in the application of magnetic flux leakage technology

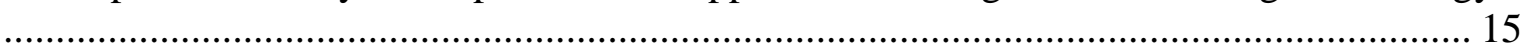

2.4.1 Sensors and magnetizer stay in close proximity of the pipe wall .................... 15

2.4.2 "A little bit of debris won't affect the magnetic flux leakage results." .............. 16

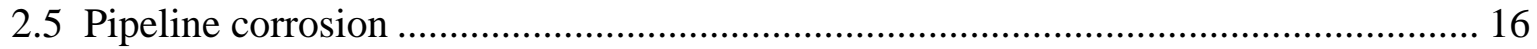

2.6 Application of magnetic flux leakage in the inspection of pipelines ..................... 17

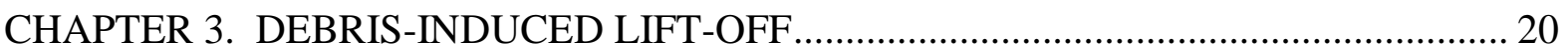

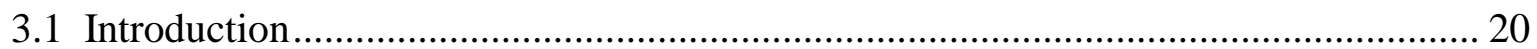

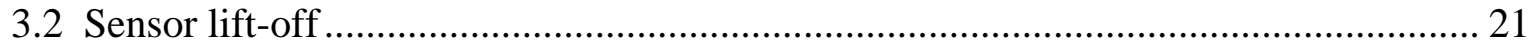

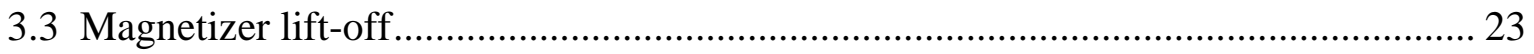

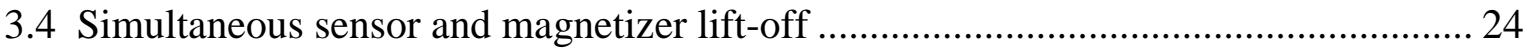


3.5 Other factors that affect magnetic flux leakage data ....................................... 24

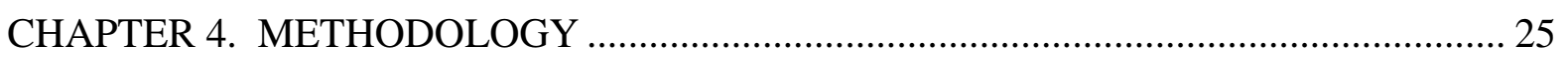

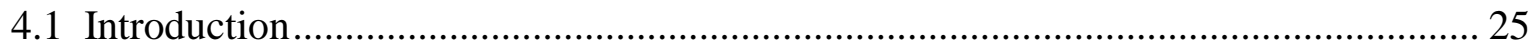

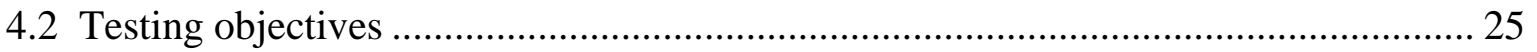

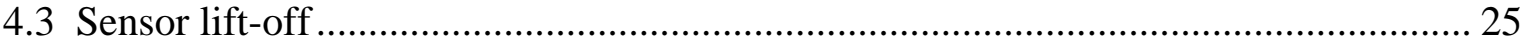

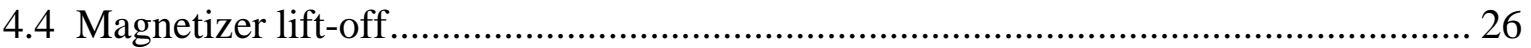

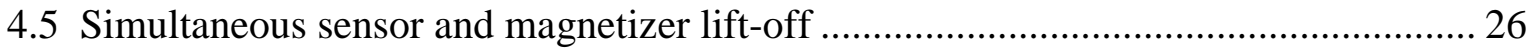

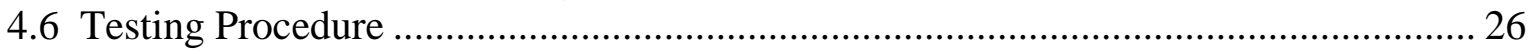

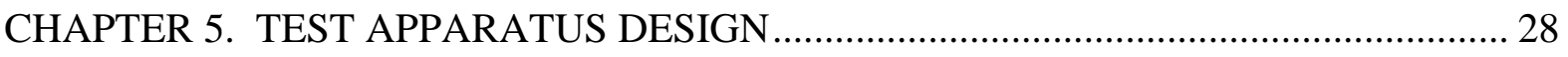

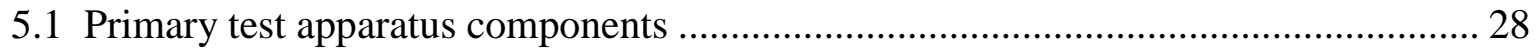

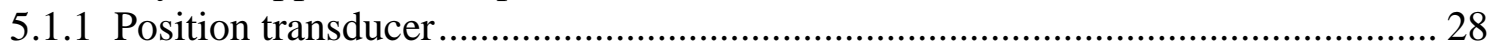

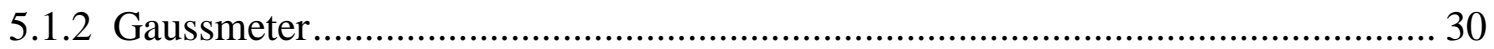

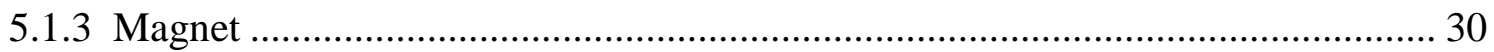

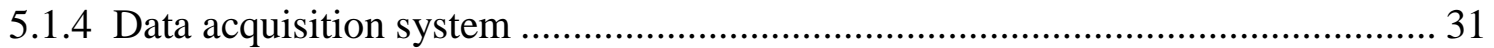

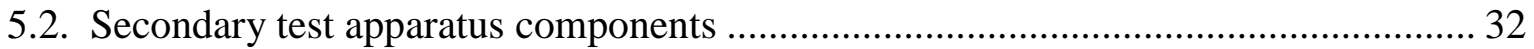

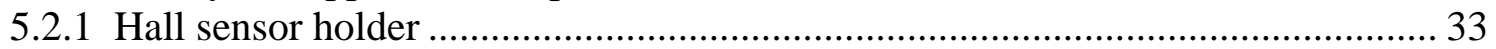

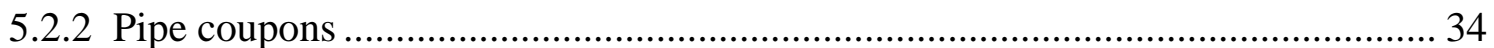

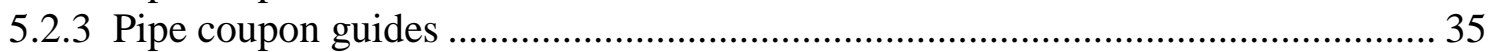

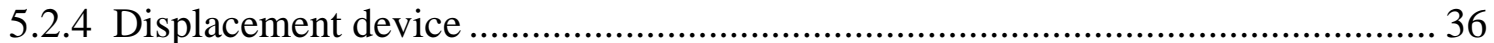

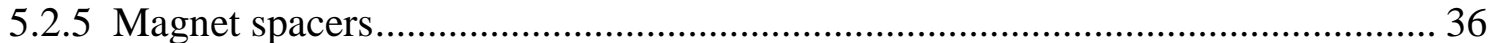

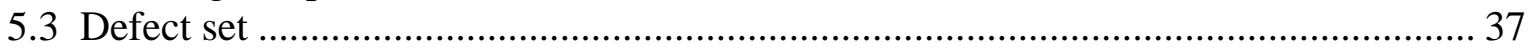

5.4 Test apparatus overview ............................................................................ 38

CHAPTER 6. ERROR ANALYSIS ........................................................................ 39

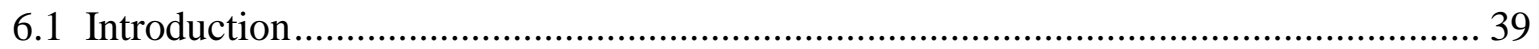

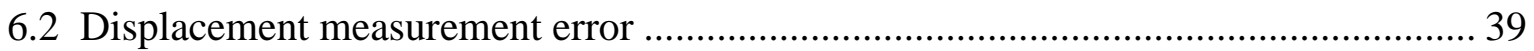

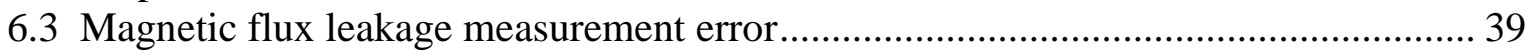

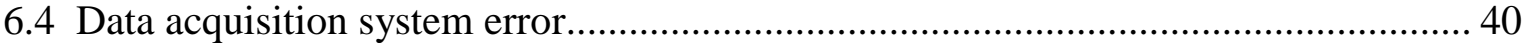

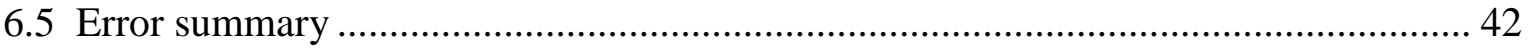

CHAPTER 7. RESULTS ....................................................................................... 44

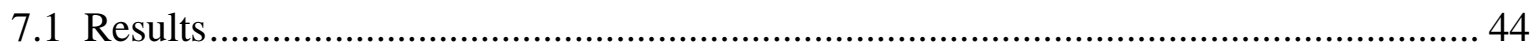

7.1.1 Lift-off (sensor, magnetizer, and both) causes a reduction in both the measured peak and baseline magnetic flux leakage amplitude for all defect types........... 44

7.1.2 Lift-off of sensors and magnet simultaneously produces the greatest reduction in measured peak magnetic flux leakage amplitude for all defect types examined.45

7.1.3 Defect geometry has an influence on the amount of reduction in measured peak magnetic flux leakage amplitude produced by lift-off................................. 45

7.1.4 Sensor lift-off has a substantially greater effect on the peak magnetic flux leakage amplitude than magnetizer lift-off of the same magnitude........................... 45

7.1.5 Low levels of sensor lift-off have a significant effect on the measured peak flux

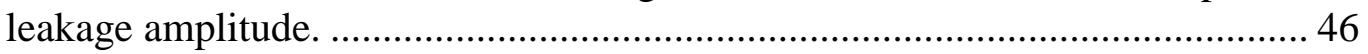

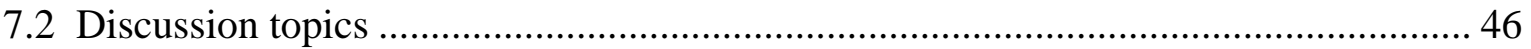

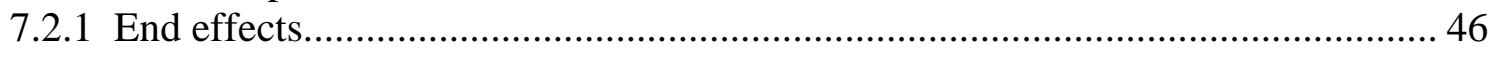


7.2.2 Change in baseline flux leakage measurements as compared to GRI results ....... 47

7.2 .3 Analog output of the gaussmeter .......................................................... 47

CHAPTER 8. CONCLUSIONS \& RECOMMENDATIONS ...................................... 48

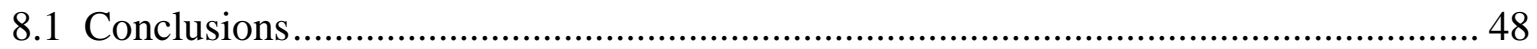

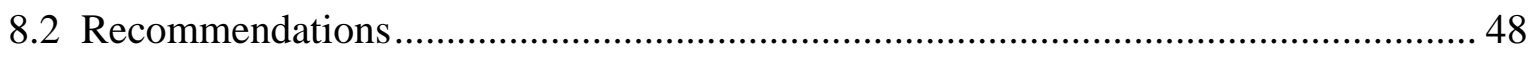

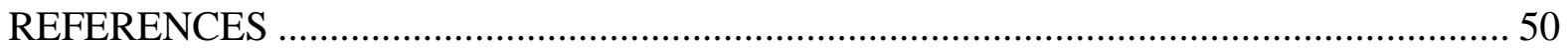

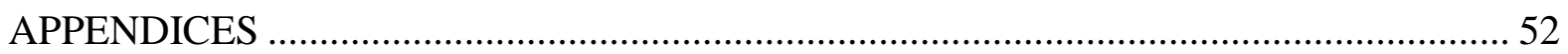

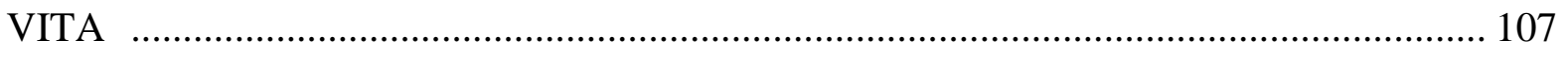




\section{LIST OF FIGURES}

Figure 1 - Requirements for anomaly detection and characterization ............................. 2

Figure 2 - Flux leakage versus magnetization level (courtesy of GRI) [8] ......................... 3

Figure 3 - Flux leakage versus magnetization level.................................................. 3

Figure 4 - Finite element modeling of flux leakage signal at various levels of sensor lift-off

(Courtesy of GRI) .............................................................................. 5

Figure 5 - Flux leakage signal at various levels of simultaneous sensor and magnetizer lift-

off (Courtesy of GRI) .................................................................................. 5

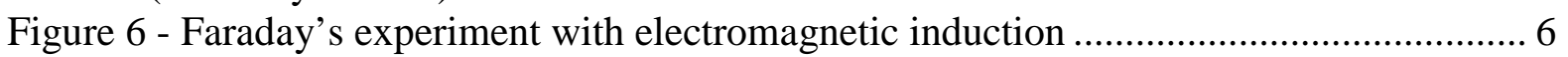

Figure 7 - Debris accumulation in a natural gas pipeline [16] .................................... 8

Figure 8 - Debris affects magnetization and measurement stages [17] .......................... 8

Figure 9 - Magnetic flux leakage inspection tool [Courtesy of PII] .................................. 9

Figure 10 - "Compression" of the magnetic flux leakage signal with lift-off of magnetizer

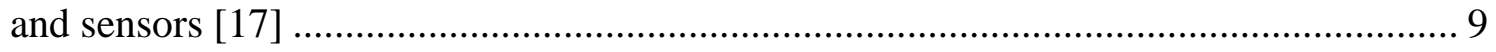

Figure 11 - Flux leakage signal from a pipeline defect (Courtesy of GRI) [8] .................... 11

Figure 12 - Baseline and peak flux leakage amplitudes [17] ..................................... 12

Figure 13 - Typical magnet and sensor arrangement .............................................. 13

Figure 14 - Picture of an actual magnetizing assembly (Courtesy of PII) [18] ................... 14

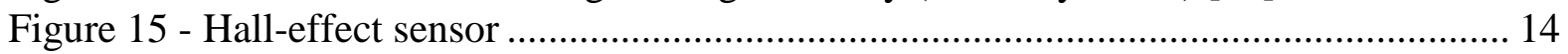

Figure 16 - ID/OD sensor array from a high-resolution MFL inspection tool (Courtesy of PII)

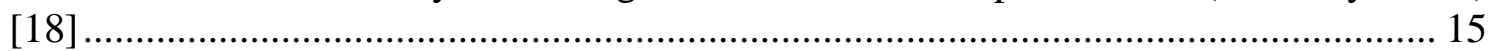

Figure 17 - Data acquisition and memory module (Courtesy of PII) [18] ....................... 15

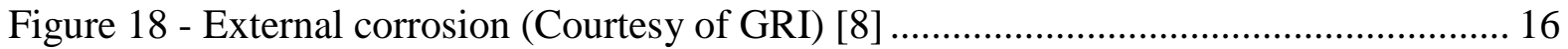

Figure 19 - Internal corrosion at 6 o'clock ...................................................... 17

Figure 20 - High-resolution magnetic flux leakage inspection tool (Courtesy of PII) [18] ... 17

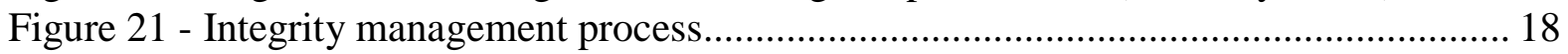

Figure 22 - Data display software (Courtesy of PII) [18] ............................................. 19

Figure 23 - Magnetizer and sensor lift-off by debris accumulation (end view) [17]........... 20

Figure 24 - Sensor head angle with/without debris-induced lift-off.............................22

Figure 25 - Sensor head angle ..................................................................... 22

Figure 26 - Additional lift-off created by sensor head angle ....................................... 23

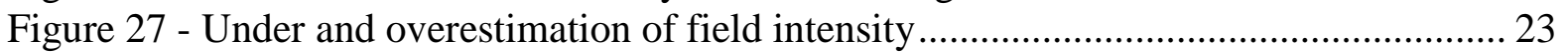

Figure 28 - Magnetizer and sensor lift-off by debris accumulation (side view).................. 24

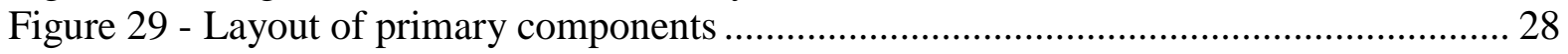

Figure 30 - Position transducer mounted to vice (side view) .................................... 29

Figure 31 - Position transducer cable fully extended.............................................. 29

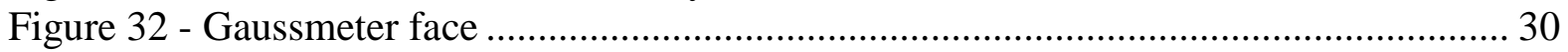

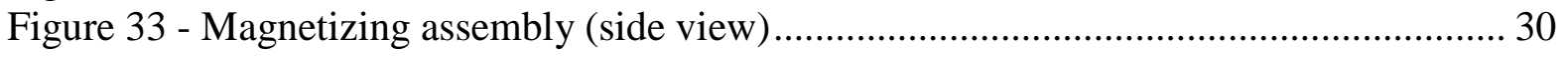

Figure 34 - Magnetizing assembly (end view) ........................................................ 31

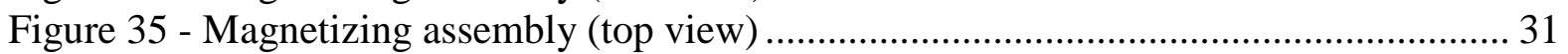

Figure 36 - Data acquisition system and inputs .................................................... 32

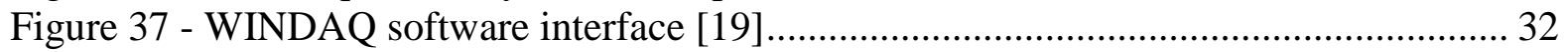

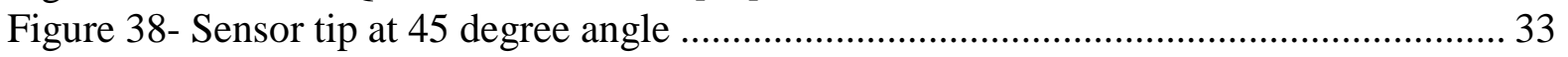

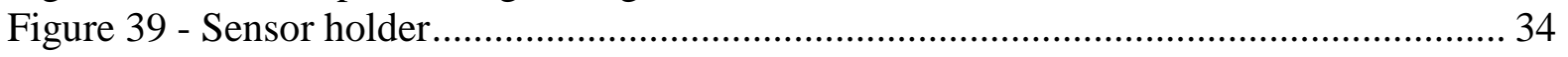




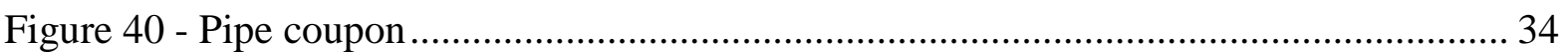

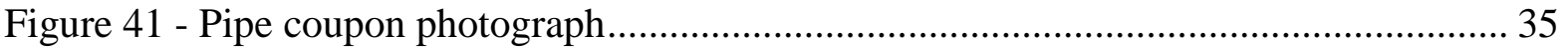

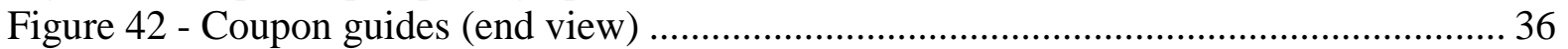

Figure 43 - Magnet spacing devices (side and top view)........................................ 37

Figure 44 - Test apparatus overview ........................................................................ 38

Figure 45 - Probe tip lift-off measurement of 0.5 inch ................................................. 40

Figure 46 - Flux leakage and distance measurement error ....................................... 42

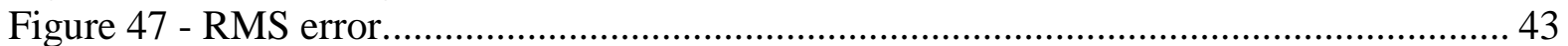

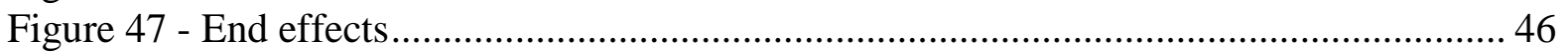




\section{LIST OF TABLES}

Table $1-12$ indicators that debris has affected magnetic flux leakage data [17] .............. 10

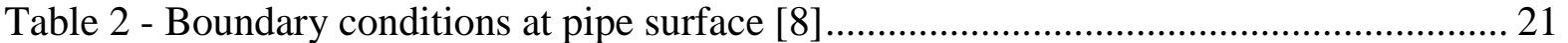

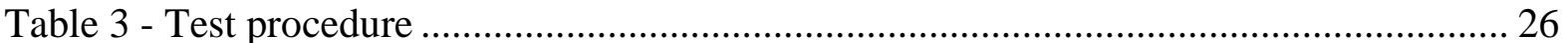

Table 4 - Voltages corresponding to anomaly axial length......................................... 26

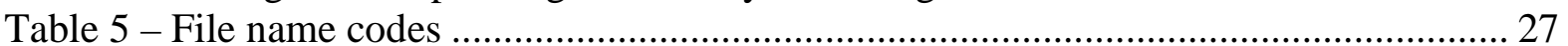

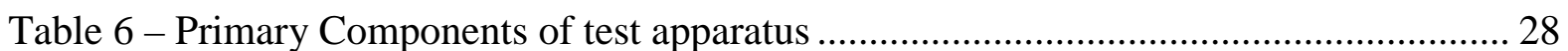

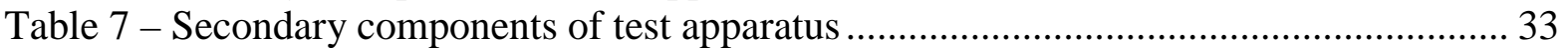

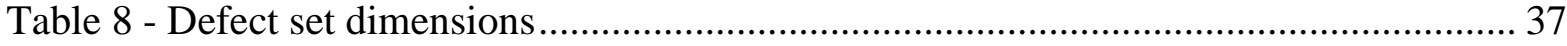

\section{LIST OF SYMBOLS}

$\begin{array}{ll}\alpha & \text { angle } \\ \text { I } & \text { current } \\ \text { G } & \text { Gauss } \\ \text { V } & \text { Volt } \\ \text { H } & \text { Magnetic field } \\ \text { B } & \text { Magnetic flux density }\end{array}$

\section{LIST OF SUBSCRIPTS}

$\begin{array}{ll}\text { A } & \text { Additional } \\ \mathrm{C} & \text { Control } \\ \mathrm{CC} & \text { Control current } \\ \mathrm{H} & \text { Hall } \\ \mathrm{N} & \text { Normal } \\ \mathrm{T} & \text { Tangential }\end{array}$

\section{LIST OF ABBREVIATIONS}

MFL Magnetic Flux Leakage

GRI Gas Research Institute

PII Pipeline Integrity International

MSP Maximum Safe Pressure

MAOP Maximum Allowable Operating Pressure

NDT Nondestructive Testing

PT Position Transducer

RMS Root Mean Squared

ID/OD Inner Diameter/Outer Diameter 


\section{CHAPTER 1. INTRODUCTION}

\subsection{INTRODUCTION}

Magnetic flux leakage (MFL) is a non-destructive testing method used to inspect ferrous materials. It is the most common method of inspecting natural gas pipelines. It is also used to inspect pipelines transporting other products such as diesel, jet fuel, and oil.

Maintaining the integrity of these pipelines is an ongoing process. This continual effort is integral to the pipeline operator's goal of maintaining safe, reliable, and efficient pipeline operations. MFL inspection is an efficient and effective method of inspecting pipelines. Given the wide range of conditions that MFL inspection vendors must accommodate in order to inspect the pipelines, they are still able to provide a valuable service. The data they provide to the pipeline operator is vital to achieving the pipeline operator's goal.

It is generally accepted that all pipelines contain some quantity of debris and that debris can potentially have a negative impact on MFL data. However, the amount of debris that produces problems in MFL results is not clear. A better understanding of how MFL inspection tools are affected by pipeline debris may help improve the operator's ability to maintain the integrity of their pipelines.

\subsection{PROBLEM STATEMENT}

There are a multitude of factors that can impact MFL inspection data. Many of these have already been documented. Pipeline debris has not traditionally been regarded as a serious threat to the acquisition of quality MFL inspection data. However, pipeline debris (even in small amounts) can cause a host of undesirable effects [6,17]. Debris-induced liftoff of the sensors, magnetizer, and both simultaneously is of particular interest and will be further examined. It is the intent of this research to probe this lift-off phenomenon and to conduct experiments to better understand the effect that debris-induced lift-off has on MFL inspection results.

\subsection{LITERATURE REVIEW}

Emphasis is given to references pertaining to pipeline cleanliness and lift-off as it relates to MFL inspection technology. This literature review section presents a brief, selective review of the most applicable references.

Christopher Kershaw [6] provides a description of the difference between operational cleanliness of a pipeline as compared to inspection cleanliness. Kershaw says that the presence of wax, scale, and dust, even in relatively small quantities, will generally downgrade the available data from an inline inspection tool even though the pipeline cleanliness level is acceptable from an operational point of view. For this reason, he says it makes sense to properly clean any suspect pipelines.

Kershaw conveys an industry sentiment that an inline inspection tool operating on the MFL principle is the most efficient cleaning tool. This is due to the tool's steel brushes, its length, and weight. This combination ensures that much of the debris it liberates from the pipe wall will be pushed ahead of the tool. Therefore, pipelines thought to be clean because of regular running of cleaning tools can generate significant amounts of debris during an 
MFL inspection. This occurs because regular cleaning tools are not as aggressive as an MFL tool and rarely removes all of the debris from the pipeline system.

Kershaw also says that there can be no argument that from an inspection point of view that a clean pipeline will give better and more accurate inspection results than a pipeline containing debris. If the debris is loose, not adhering to the pipe wall, and is in low quantities, the inspection tool will most likely push the debris ahead of it as it proceeds through the pipeline. However, if the quantity is high there is a distinct possibility that some of the debris will eventually be over-ridden by the inspection tool which may reduce or eliminate the ability to inspect the pipe wall. This problem is more pronounced on longer pipelines.

Kershaw states that if the debris is firmly attached to the pipe wall, the situation is far more serious from an inspection point of view. He says that a thin layer may not cause too many problems, but a thick layer could render the pipe wall uninspectable at this location. In addition, as the inspection tool progressively removes more debris from the pipe wall and pushes it ahead, the risk of over-riding the debris accumulation increases which again reduces or eliminates inspection capability.

This paper concludes with a description of several types of enhanced cleaning tools and gives examples of their use. Also stressed is the importance of gradually increasing the aggressiveness of the pipeline cleaning program. This ensure that large accumulations of liberated debris do not form which could cause the cleaning tools to become stuck.

Nestleroth, et al [8] provide a very thorough and comprehensive review of the MFL concept and its application in the natural gas transportation industry. This particular document is a valuable resource for anyone involved in MFL inspection of pipelines. With a thorough understanding of how MFL tools operate it becomes evident where pipeline debris may affect its operation.

Clearly defined are the four steps or requirements for anomaly detection and characterization. They are magnetization, leakage, characterization, and analysis (Figure 1).

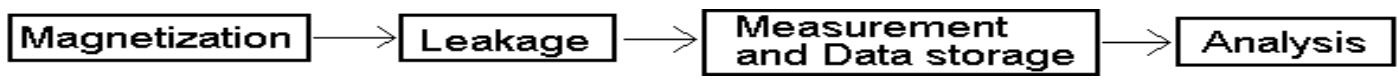

Figure 1 - Requirements for anomaly detection and characterization

Magnetization of the pipe wall to saturation is essential for anomalies to be reliably and accurately detected and characterized. Figure 2 shows how the level of magnetization affects the resulting flux leakage amplitude. It is obvious that stronger levels of magnetization produce stronger flux leakage signals. 


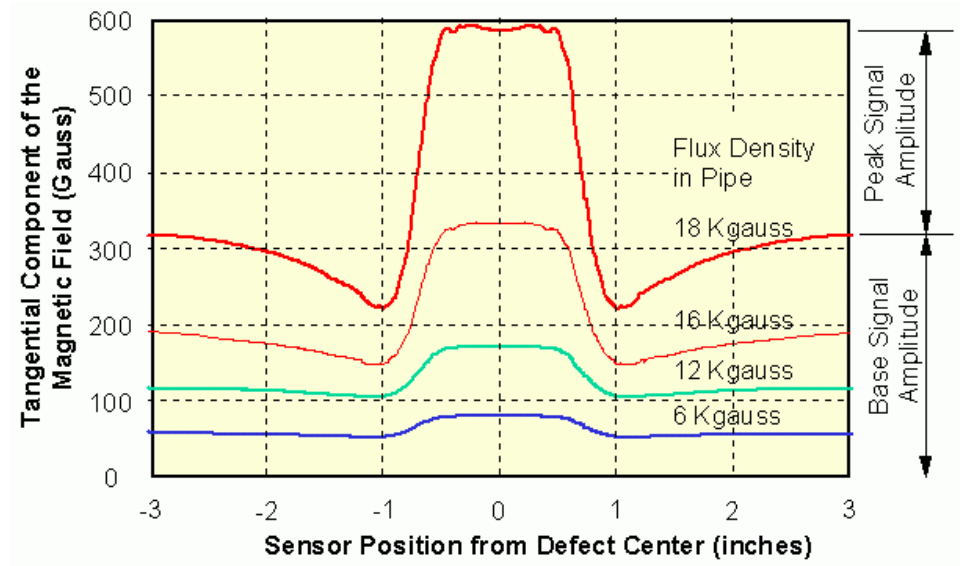

Figure 2 - Flux leakage versus magnetization level (courtesy of GRI) [8]

A strong induced magnetic field is desired because it minimizes noise or error in the acquired signal that result from variations in the permeability of the steel (due to variations in carbon content, alloying elements, and impurities). Strong magnetic fields also improve sensitivity of the sensors to changes in the MFL field.

In order for flux leakage to occur two conditions must be satisfied. First, the induced magnetic field must be strong enough to magnetically saturate the pipe. Figure 3 graphically depicts the effect of magnetization level on the resulting flux leakage field. Secondly, a reduction in flux carrying capacity must occur. This is typically satisfied by a change in geometry or magnetic properties.

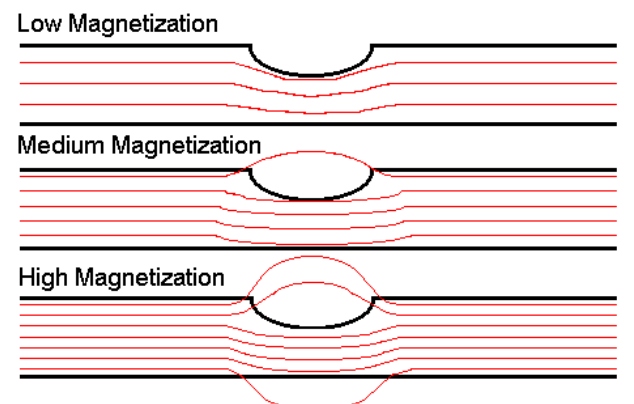

Figure 3 - Flux leakage versus magnetization level

If saturation is not achieved, the remaining wall thickness may be enough to pass the induced magnetic flux without creating any additional flux leakage. When the tool encounters heavy-wall pipe as is typical at road crossings, the magnetization levels drop significantly because of the additional flux carrying capacity of the thicker-walled pipe. This reduction in magnetization level also occurs at girth welds, tees, taps, and saddles where the available metal mass increases. Detection and characterization of defects in these areas is extremely difficult.

When an MFL inspection takes place there are a number of variables which must be measured and stored in order for the data to be useful. These include, but are not limited to, MFL magnitude, position, and ID/OD location. The sensor system measures the MFL fields. An odometer wheel provides the displacement data which is used to determine a defect's location along a pipeline and also to estimate the axial length of detected defects. The 
ID/OD sensors determine the location of the detected anomaly with respect the inside or outside surface of the pipe wall.

Once the data has been retrieved from the inspection tool, the analysis process begins. Analysis is the process of estimating the geometry (length, width, and depth) of an anomaly from the retrieved MFL data $[2,8,10]$. As stated before, the quality of the analysis is dependent upon the accuracy of the previous steps. Therefore, if the magnetization, leakage, measurement steps are affected this has a corresponding effect on the resulting analysis.

Length, width, and depth are important parameters in the assessment of an anomaly's severity and its impact on a pipeline's integrity. Initially, an anomaly's depth is inferred from the change in flux leakage signal from baseline level to maximum flux leakage amplitude. The length of an anomaly is determined by noting the distance traveled during the positive departure of the MFL signal from baseline level. Finally, the width of an anomaly is determined by counting the number of sensors that experience a predetermined change in flux leakage signal. Knowing that each sensor accounts for some circumferential distance around the pipeline, the circumferential width of an anomaly can be determined.

The analysis phase of the MFL inspection process takes this raw data and applies advanced data analysis techniques to estimate the geometry of anomalies. Neural networks and other proprietary solutions have been developed to assist in the analysis of this data. The estimated length and width of an anomaly affects the final depth estimation of the anomaly.

This paper clearly points out the fact that MFL inspection technology is not infallible. It does have limitations which must be recognized and understood to fully appreciate the results it provides. It points out that there are a multitude of factors, all of which are not known or fully understood, that can influence the acquired MFL signal. Even with the best of conditions, detection and characterization of all anomalies is not guaranteed.

The Gas Research Institute (GRI) conducted finite element analysis on the effect of sensor lift-off on the acquired MFL signal. Figure 4 shows the results of finite element modeling of sensor lift-off which clearly illustrates a degradation in measured peak MFL amplitude with increasing sensor lift-off. This analysis exemplifies the importance of the sensors being in close contact with the pipe wall. It shows that the ability to estimate the depth of defects decreases with increasing sensor lift-off. Depending on the defect geometry, a small change in lift-off may cause a large change in signal amplitude. This could result in erroneous interpretations of the true severity of pipeline defects. 


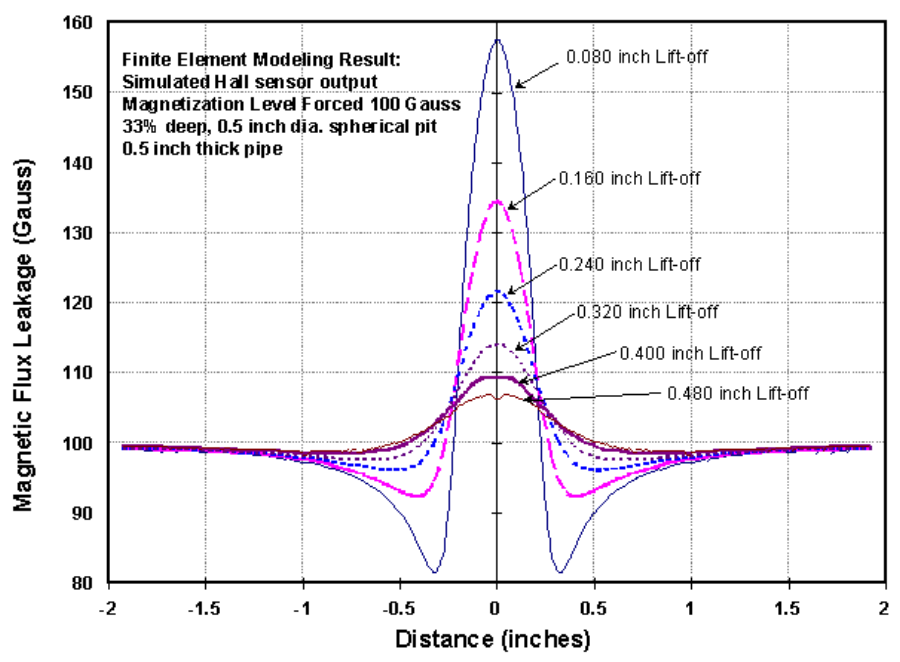

Figure 4 - Finite element modeling of flux leakage signal at various levels of sensor lift-off (Courtesy of GRI)

GRI also tested internal pipeline liners to determine their effect on the acquisition of MFL data [8]. Internal pipe liners are a method of pipe rehabilitation that is being evaluated for future commercial use. GRI's data clearly shows degradation in the acquired MFL signal with increasing lift-off (of both sensors and magnetizer). Figure 5 shows that, the air (gas)coupled field increases as indicated by the higher flux leakage level of the baseline. Also evident is a noticeable decrease in the peak amplitude of the MFL signal generated by the corrosion defect set. At the far right of the curve (at 138 inch), the 20\% deep corrosion defect is nearly indistinguishable at $20 \mathrm{~mm}$ (0.48 inches) of lift-off (both sensor and magnetizer). This clearly exemplifies the possibility of defects going undetected or underestimated as a result to simultaneous sensor and magnetizer lift-off.

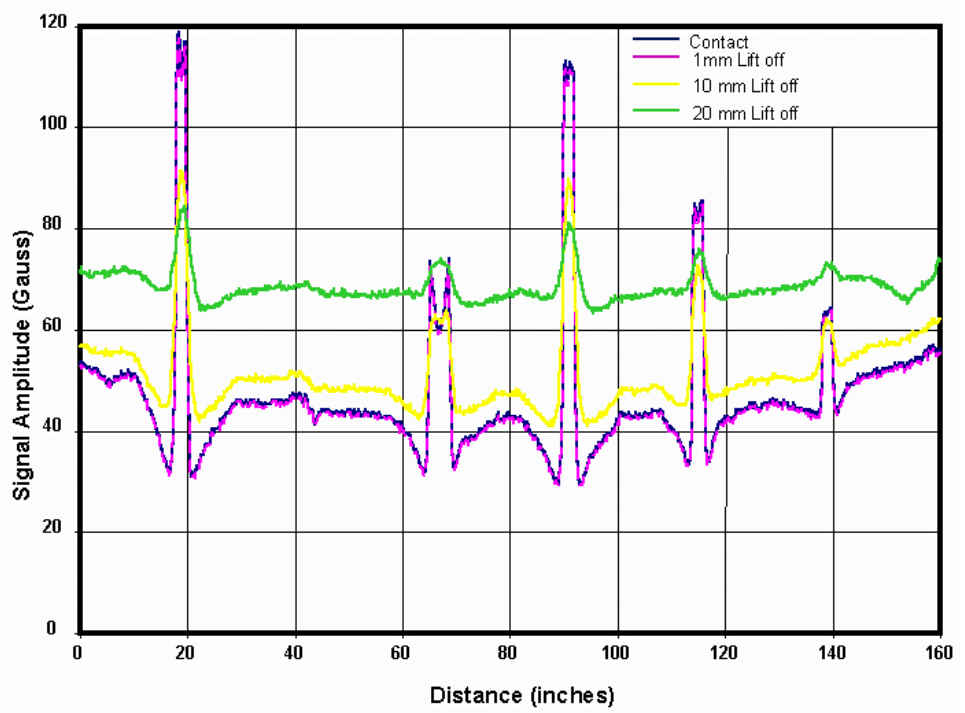

Figure 5 - Flux leakage signal at various levels of simultaneous sensor and magnetizer lift-off (Courtesy of GRI) 
Payne [9] points out the fact that the quality of MFL inspection data is dependent on the cleanliness of the pipeline. Payne states that debris affects the sizing accuracy of corrosion anomalies and also has the potential to cause the inspection tool to become stuck, necessitating a costly pipeline shutdown to extract it. Payne says that most pipeline operators normally run one or two pipeline cleaning tools prior to an MFL inspection. He goes on to say that in some cases this may be adequate, but it is not in most cases. Payne says the cleaner the pipeline, the more accurate the MFL inspection results. Also described are the various pipeline cleaning tools that may be used to prepare a pipeline for inspection.

Tiratsoo [13] highlights the importance of pre-inspection cleaning and provides criteria to help the operator determine when a pre-inspection cleaning program is required. One of the objectives of pre-inspection activities is to determine if debris is present. If it is found to be present, the goal is then to remove it from the pipeline. However, the pipeline operator's debris removal attempt is, in many cases, fulfilled by running 1 or 2 cleaning tools. This is not sufficient to attain inspection cleanliness. In addition, this does not produce conclusive results because the effectiveness of the cleaning tools is determined by consequential evidence (i.e. the amount and quality of debris that is accumulated in the receiver and the physical condition of the cleaning tools). Pipelines that are known to contain debris should have an effective pipeline cleaning program executed in preparation for the MFL inspection.

Tiratsoo further acknowledges that the condition of the internal pipe wall surface has an effect on MFL inspection data. He states that lift-off of the magnetic brushes causes a reduction in the applied magnetic field and that lift-off of the sensors reduces its ability to accurately measure flux leakage. In short, lift-off of the magnetizer and sensors reduces the level of detection.

Tiratsoo indicates that the level of inspection confidence and accuracy demanded by today's pipeline operators require advanced inspection tools to examine every square inch of pipe wall. Millions of dollars are allocated to maintain the integrity of these pipelines, so the requirement for accuracy of the MFL data is at an all time high. Therefore, pipeline cleaning activities are a very important link in the chain leading to high quality inspection data.

The Nondestructive Testing Handbook (NDT) [2] is a valuable resource for the history, concept, and application of eddy current, magnetic flux leakage, and microwave nondestructive testing methods. It is revealed that electromagnetic testing is the most ancient of nondestructive testing methods. As early as the $6^{\text {th }}$ century BC, Thales of Miletus first recorded that rubbing amber induces a state in which the amber would attract other light objects. Circa $1200 \mathrm{AD}$, the use of a magnetic compass was reported in China.

In 1831, Joseph Henry and Michael Faraday discovered electromagnetic induction. Faraday observed that when a primary circuit is connected to a voltaic battery in close proximity to a secondary circuit, a current is produced in the secondary circuit (Figure 6). This observation is the basis for the development of many nondestructive testing techniques.

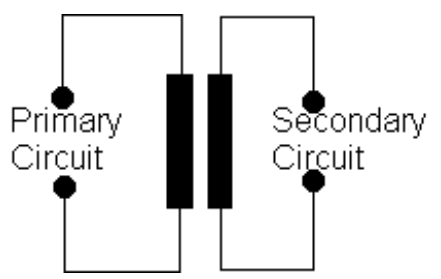

Figure 6 - Faraday's experiment with electromagnetic induction 
Faraday's work has also been the inspiration upon which many other advances were developed. These include Morse's telegraph, Bell's telephone, and Edison's improvements on the telegraphic, telephonic, fire alarm, and stock ticker communications systems.

James Clerk Maxwell produced a comprehensive compilation of equations know as Maxwell's Equations. These equations mathematically represent all of the present knowledge of electromagnetic circuits. Since the advent of Maxwell's equations there have been no significant discoveries which extend the current understanding of electromagnetic theory.

The NDT handbook goes on to explain the theory behind the MFL inspection methodology and provides many examples of its use. The NDT handbook states that in order to obtain stable detection of discontinuities, sensor lift-off must remain constant. Also discussed is the fact that the dimensions of the detected anomalies cannot be accurately determined from the acquired MFL data. There are a multitude of parameters, all of which are not fully understood, that contribute to the final signal. Therefore the signal shape, alone, is not an effective method of determining the severity of an anomaly.

Valentine [15] provides an introduction to the online chemical cleaning process. He describes a natural gas transmission company that experienced difficulty in acquiring reliable MFL data for the entire length of a pipeline because of excessive debris accumulations. This debris consisted primarily of salt and black powder. Prior remedial work (pipeline segment replacements and drip removals) confirmed the presence of large amounts of debris which prompted several unsuccessful pipeline cleaning operations using dry mechanical cleaning methods. Still, reliable MFL inspection data for the entire length of pipeline was unobtainable because of excessive debris accumulations.

This company realized that a more effective pipeline cleaning solution was required, so the online chemical cleaning method was used. This pipeline cleaning program resulted in the removal of tons of debris from the pipeline system. After the cleaning process, the pipeline was re-inspected. Not only was complete and reliable MFL inspection data acquired, but the pipeline also experienced a significant gain in efficiency. These gains in efficiency quickly paid for the cost of the online chemical cleaning operation. Valentine goes on to say that the increase in the quality of the inspection data provides a collateral increase in the quality of the integrity assessment results and decreases the likelihood that serious defects are missed or undersized.

Valentine [16] explains how debris affects the operation of the geometry inspection tool. A geometry tool is an inline inspection device that measures and records the internal geometry of the pipeline. This data is important in assessing the MFL inspection tool's likelihood of traversing the pipeline unimpeded. Dents, buckles, ovalities, wrinkle bends, and debris accumulations have the potential to cause internal diameter restrictions. Depending on the severity of the detected obstruction, the MFL inspection may be aborted or at least postponed to investigate, effectively clean the pipeline, or perform remedial work if necessary. Figure 7 shows an accumulation of debris that was discovered in a natural gas pipeline as a result of a geometry inspection. 


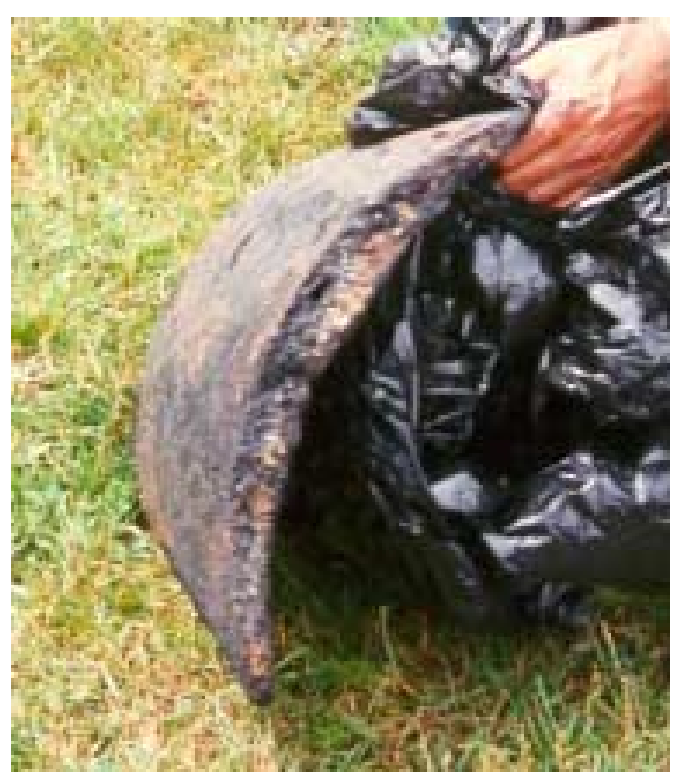

Figure 7 - Debris accumulation in a natural gas pipeline [16]

Debris has been found to degrade the geometry tool's sensitivity to changes in the internal geometry of the pipeline. Debris-induced lift-off prevents the geometry tool from contacting the internal pipe surface. This can lead to the inability to detect wall thickness changes and other pipeline features.

Geometry inspection tool data can also provide a means of assessing the cleanliness of a pipeline. This article points out the fact that cleanliness is a significant factor in the quality of the acquired data. The same debris that affects geometry inspection tools can also affect the operation of MFL inspection tools. Effective maintenance and pre-inspection cleaning programs can eliminate (or at least reduce) the impact of debris on the acquired inspection results.

Valentine and Luedke [17] provide a review of the theory behind MFL testing how debris affects the MFL inspection results. They also point out the fact that debris directly affects the magnetization and measurement steps of the MFL testing process (Figure 8).

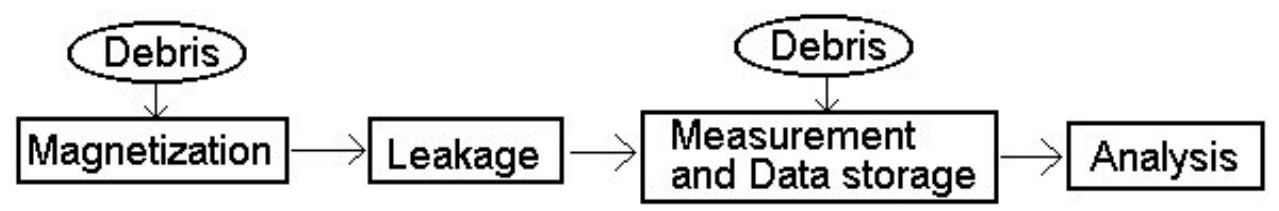

Figure 8 - Debris affects magnetization and measurement stages [17]

Figure 9 reveals where debris may impact the operation of an MFL inspection tool. Debris may cause lift-off of the magnetizer which can affect its ability to saturate the pipe wall and produce strong flux leakage fields. Debris can also cause lift-off of the sensors which affects its ability to accurately measure the flux leakage field and determine the location (internal/external) of defects. Odometer wheel slippage and jamming is also possible when debris is present which can cause inaccurate measurement of displacement. 


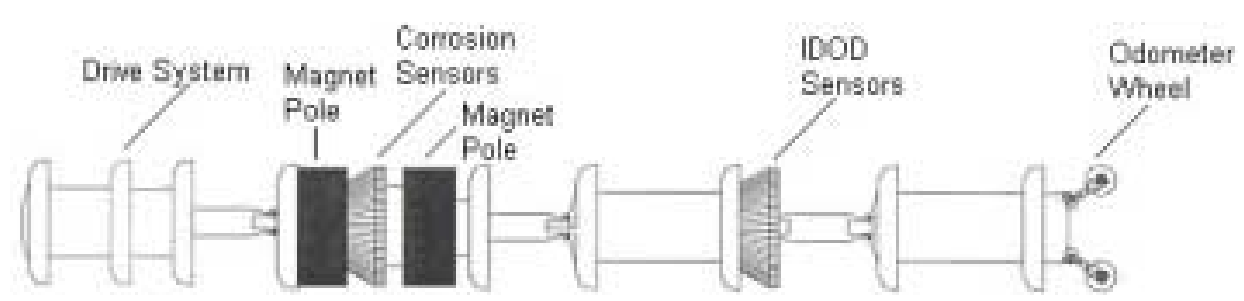

Figure 9 - Magnetic flux leakage inspection tool [Courtesy of PII]

All of these effects can lead to inaccurate assessments of the severity of pipeline defects.

Valentine and Luedke go on to describe the primary mechanisms by which debris affects MFL tool operation. These are inspection tool damage, velocity excursions, and liftoff (magnetizer, sensor, or both). All of these mechanisms can occur exclusive of one another or at the same time. These mechanisms serve to degrade the quality of the acquired MFL data.

Valentine and Luedke's work also produced Figure 10 which summarizes the findings of GRI's testing and explains the effect of simultaneous sensor and magnetizer liftoff on the acquired MFL data. The compression of the flux leakage signal can affect the analysis of the acquired MFL signal, resulting in inaccurately sized or missed anomalies.

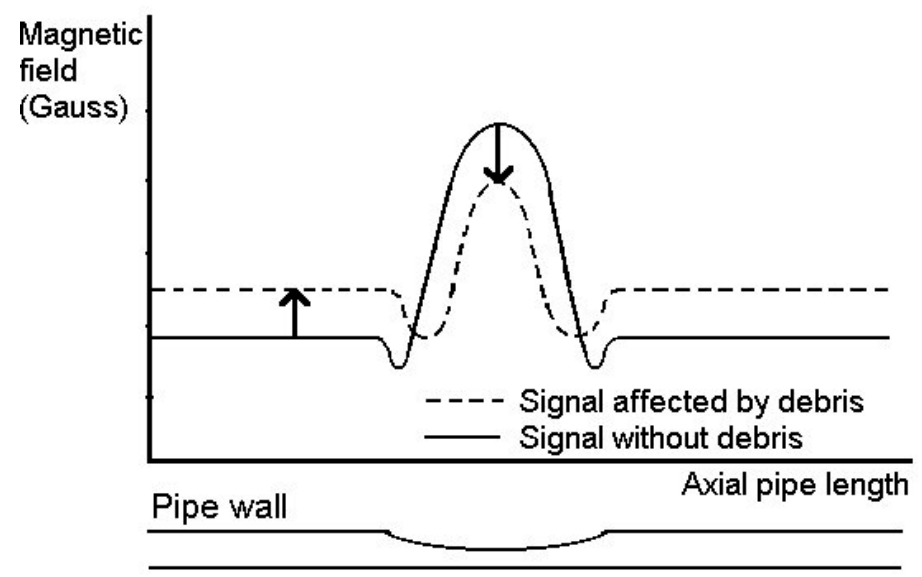

Figure 10 - "Compression" of the magnetic flux leakage signal with lift-off of magnetizer and sensors [17]

Lift-off of the magnetizer results in an increase in the gas-coupled field which is indicated by the increase in baseline MFL level. In addition, the amplitude of the measured flux leakage is reduced for two reasons. First, since the amount of flux transferred into the pipe wall is reduced, the amplitude of the resulting flux leakage field also is reduced. Second, lift-off of the sensors impairs their ability to measure the true flux leakage magnitude. The end result is an MFL signal that is not representative of the true flux leakage field.

Valentine and Luedke's also compiled a list of 12 indicators which may be used to determine whether the acquired MFL data has been affected by debris. These are provided in Table 1. 


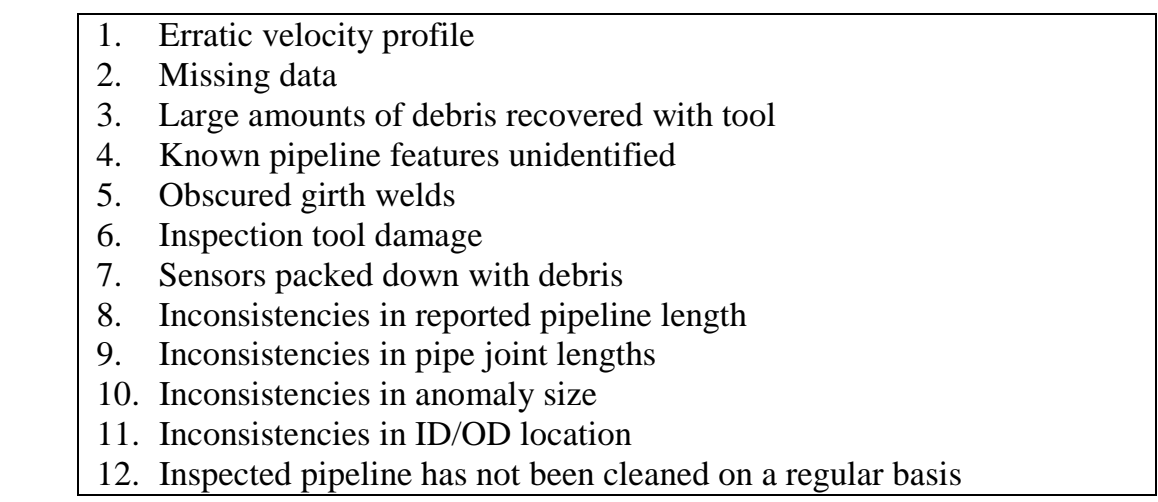

Table 1 - 12 indicators that debris has affected magnetic flux leakage data [17]

The greater the number of indicators present in a section of pipeline, the greater the probability that debris has impacted the acquired MFL data [17]. If it is determined that the MFL data has been affected, the operator should clean the pipeline using the appropriate method and re-inspect the pipeline. This will minimize the probability that injurious defects have gone undetected or underestimated as a result of debris induced lift-off.

\subsection{OBJECTIVES}

The objective of this research is to determine the effect of debris-induced lift-off on MFL inspection results. In order to achieve this objective, the following issues are addressed:

1. Perform a literature search to locate applicable references which provide insight into the effects of debris on MFL inspection results.

2. Conduct experiments to qualitatively determine what effect the debris-induced lift-off has MFL results.

3. Review the acquired data, draw conclusions, and make recommendations. 


\section{CHAPTER 2. MAGNETIC FLUX LEAKAGE}

\subsection{INTRODUCTION}

There are hundreds of thousands of miles of underground pipeline in the continental United States and abroad. The majority of these pipelines are in excess of 20 years of age. During the time of their installation, they did not have the benefit of today's knowledge and experience in corrosion control techniques and materials (steel, coatings). To replace this aging infrastructure with today's materials would be cost prohibitive. Therefore, the most economical and practical solution is to control corrosion, monitor it's development, and replace corroded pipe before its integrity is compromised [17].

When the majority of pipelines were constructed, they were located in rural areas where the population density was very low. Pipeline failures, then, only threatened continuity of service for the pipeline's customers. Over the years, the population density in the vicinity of these once remote pipelines has drastically increased. This has caused increased concerns for the safety and integrity of pipelines. As a result, pipeline operators are increasingly relying upon inline inspection data as well as other types of inspections (close interval survey, leakage survey, etc.) to certify or validate the integrity of their pipelines. MFL inspection tools are an example of an inline inspection device used to inspect pipeline for defects.

\subsection{MAGNETIC FLUX LEAKAGE CONCEPT}

In its most basic form, MFL inspection involves the magnetization of a specimen and the observation or measurement of the resulting flux leakage field as shown in Figure 11 [2]. The resulting flux leakage is typically indicative of some undesirable feature (e.g. crack, geometry irregularity, and corrosion). The source of magnetization may be either permanent magnet or an electromagnet. The flux leakage detection may utilize either magnetic particles (e.g. magnetic particle inspection) or a variety of magnetic sensors.

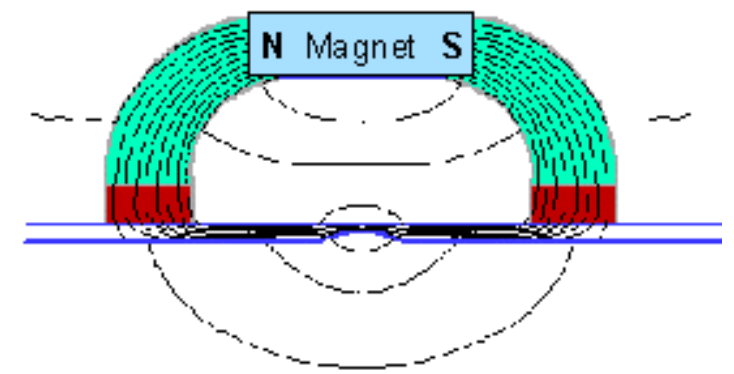

Figure 11 - Flux leakage signal from a pipeline defect (Courtesy of GRI) [8]

It is important to understand that this is an inferential measurement technique. In other words, the severity of a feature is "inferred" from the measured or observed change in the MFL magnitude. Direct measurements of the wall thickness are not acquired as is the case for ultrasonic inspections. Therefore, this technology can reliably detect metal loss regions, but it is not as reliable when it comes to estimating depth, length, and width of a corroded area [2]. 
Ultrasonic inspection tools can also be used to inspect pipelines, but there are some issues that typically preclude its use in the inspection of natural gas pipelines. Ultrasonic inspection typically requires the use of a liquid couplant. This is not a problem for liquid pipelines. However, for inspection of natural gas pipelines this creates additional handling and disposal hurdles. In addition, ultrasonic inspection requires a greater level of pipeline cleanliness because debris can prevent the reflection of the ultrasonic pulse. If the receiver cannot measure a reflected pulse, no data is collected. However, it is possible to determine where this occurs.

MFL inspection tools, on the other hand, are more forgiving of pipeline debris. Debris will not prevent the acquisition of MFL data, but it will downgrade the quality of the data $[6,8,9,16]$. The disadvantage of MFL inspection is that it is not possible to determine where the data has been affected by debris-induced lift-off and to what extent. However, MFL inspection is more robust, less expensive, and easier to apply than ultrasonic inspection. This is particularly true for the inspection of natural gas pipelines.

MFL inspection requires that a length of pipe wall be magnetized to saturation $[2,8,13,17]$. Saturation is the point at which a further increase in the applied magnetic field produces only small increases in flux density in the pipe wall. Therefore, the pipe wall, at its current thickness, can hold no more magnetic flux and will begin to "leak" magnetic flux out of the pipe wall and into the air, gas, or liquid. Therefore, a baseline MFL reading is present even where no corrosion is present (Figure 12).

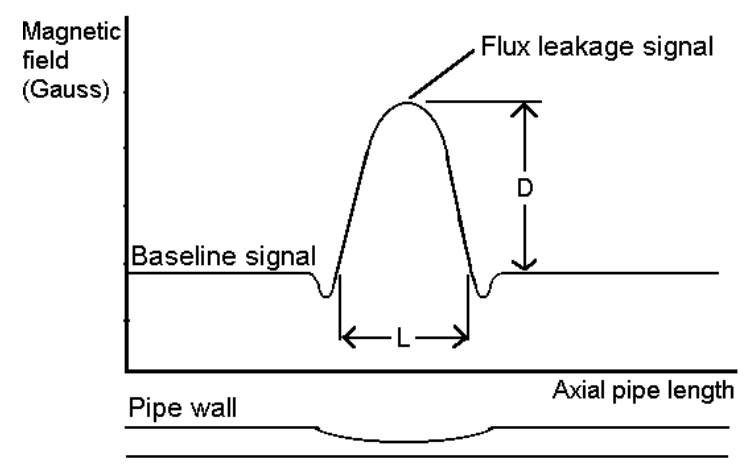

Figure 12 - Baseline and peak flux leakage amplitudes [17]

When wall thickness changes or other variations in the steel's magnetic properties are encountered, a change in the measured flux leakage signal is produced (Figure 12). This change in flux leakage field, if detected, is measured by a sensor array and stored by analog or digital means for later analysis.

Three components of the flux leakage field may be measured: tangential $\left(\mathrm{H}_{\mathrm{T}}\right)$, normal $\left(\mathrm{H}_{\mathrm{N}}\right)$ and circumferential $\left(\mathrm{H}_{\mathrm{C}}\right)$. It is most common for inspection vendors to, at the very least, measure and record either the tangential or normal component of the MFL field. From these components, the geometry (depth, length, and width) of many pipeline features and corrosion anomalies can be estimated.

Because the induced magnetic field is typically axially aligned, the circumferential component of the leakage field is commonly disregarded. Recently developed inspection tools utilizing circumferentially aligned magnetic fields make use of the circumferential component. This type of inspection tool is better able to detect and characterize axially aligned defects such as slot, seam corrosion and stress corrosion cracking [18]. 


\subsection{COMPONENTS OF AN MAGNETIC FLUX LEAKAGE INSPECTION TOOL}

The main components in any MFL inspection tool consist of drive, power, magnetization, sensor, and data recording systems. These systems are connected by flexible couplings that allow data and power transfer [8]. The inspection tool, as a whole, has hard diameter and bend radius requirements typically determined by the design of the magnetizing assembly (pole spacing) [8]. The MFL inspection tool vendor will provide these specifications. A more detailed description of these components can be found in reference 8 .

\subsubsection{DRIVE SYSTEM}

The drive system is located at the front of the inspection tool. It is typically constructed with a metal body upon which a series of urethane (or other suitable material) cups are mounted. The cups are spaced so that bypass is minimized/eliminated when the tool encounters tees or other branch connections [8]. Pressure differential is generated across these drive cups as a result of the upstream and/or downstream compressor operation. This pressure differential provides the locomotive force necessary to pull the inspection tool through the pipeline.

\subsubsection{POWER SYSTEM}

The power system provides the current required by the sensors, electromagnets (if applicable), and onboard circuitry that collects, conditions, and stores inspection data. The choice of battery type is based upon the length of the inspection run and space/shape requirements [8]. The battery must also be rugged enough to withstand he shock and vibration that is reasonably expected to occur.

\subsubsection{MAGNETIZING SYSTEM}

A system for magnetizing a length of pipe wall utilizes either electromagnets or rare earth permanent magnets (Figure 13). Electromagnets allow the magnetic field strength to be adjusted as the tool encounters changing magnetic properties and wall thickness in the steel [8]. A disadvantage of this type of magnet is that it requires electrical power which becomes a concern as the length of pipe to be inspected increases. Permanent magnets do not require an external power source and are often chosen for this reason.

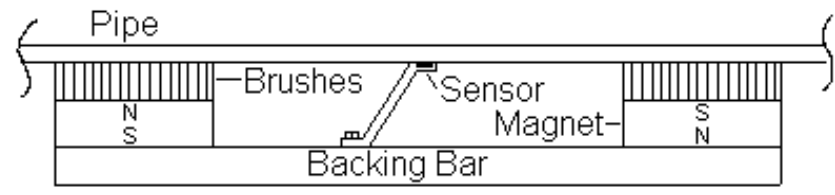

Figure 13 - Typical magnet and sensor arrangement

Steel brushes are commonly used to transfer the magnetic flux into the pipe wall. Steel brushes provide good magnetic coupling and also absorb shock and vibration. Magnetizing sections are oriented around the circumference of the pipe to provide 360 degrees of magnetic coverage. Figure 14 illustrates an actual magnetizing/sensor section from a Pipeline Integrity International (PII) high resolution MFL inspection tool. 


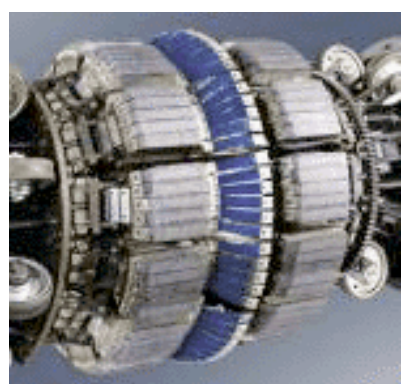

Figure 14 - Picture of an actual magnetizing assembly (Courtesy of PII) [18]

\subsubsection{SENSOR SYSTEM}

The sensor system is used to detect and measure the MFL field. Corrosion sensors are typically positioned midway between the poles of the magnetizer as shown in Figures 13 and 14. These sensors produce an electrical signal which is either proportional to the flux leakage field intensity (Hall-effect sensors) or proportional to the rate of change in flux leakage magnitude (coil sensors). From this signal, the severity of wall loss can be estimated.

The sensors in predominant use in high resolution MFL inspection tools are the Halleffect type. Their operation is independent of speed, more accurate than coil sensors, and provides the advantage of extremely compact packaging. Their operation is based on the measurement of a drift or "Hall" voltage. A control current ( $\left.\mathrm{I}_{\mathrm{CC}}\right)$ is supplied to a current carrying semi-conductor (Figure 15). When the semiconductor wafer is subjected to a magnetic field (B) which is perpendicular to the control current flow, a drift or Hall current $\left(\mathrm{I}_{\mathrm{H}}\right)$ is produced which is perpendicular to both the control current $\left(\mathrm{I}_{\mathrm{CC}}\right)$ and the magnetic field (B) being measured. The Hall voltage $\left(\mathrm{V}_{\mathrm{H}}\right)$, measured across the semi-conductor, is proportional to the strength of the magnetic field. When calibrated against a known magnetic field strength this device can be used to accurately and reliably measure the strength of an unknown magnetic field $[2,8,21]$.

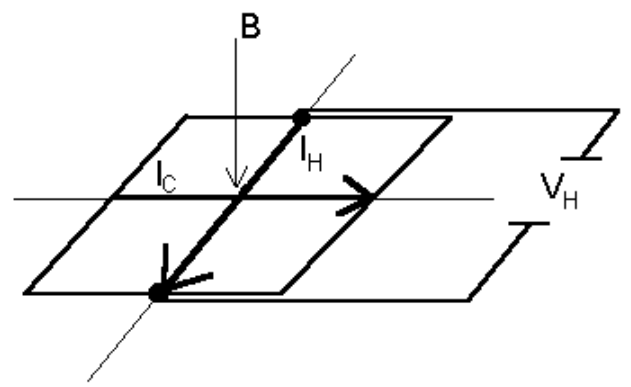

Figure 15 - Hall-effect sensor

Another set of sensors is used to determine whether the detected signal is located on the inside or outside surface of the pipe. These are commonly called the "Inner Diameter, Outer Diameter (ID/OD) sensors" or simply "discriminators." They may be eddy current, Hall-effect, or active field coil sensors. These sensors may be integrated into the design of the corrosion sensor head or may be located behind the main corrosion sensor array as shown in Figure 9. Figure 16 illustrates an ID/OD array from an actual high-resolution MFL inspection tool. 


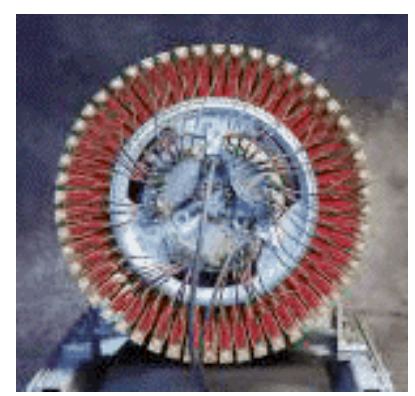

Figure 16 - ID/OD sensor array from a high-resolution MFL inspection tool (Courtesy of PII) [18]

\subsubsection{DATA CONDITIONING, ACQUISITION, AND STORAGE SYSTEM}

During a typical MFL inspection extremely large amounts of data are produced and must be stored for later retrieval and evaluation. This data can either be stored on analog or digital media. The digital format is more robust than the analog and allows additional features such as error checking and data compression [8]. The data recording module must be designed to be rugged enough to withstand the pressures, shock, and vibration typical of travel through a subterranean pipeline. Figure 17 shows a data acquisition and storage module from a PII MFL inspection tool.

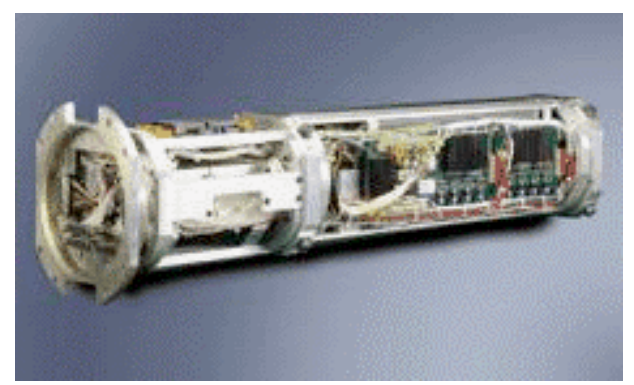

Figure 17 - Data acquisition and memory module (Courtesy of PII) [18]

\subsection{PIPELINE INDUSTRY ASSUMPTIONS IN THE APPLICATION OF MAGNETIC FLUX LEAKAGE TECHNOLOGY}

Close proximity of the sensors and magnetizer to the pipe wall is usually taken for granted. Yet in most pipelines this is not be the case. Debris, both loose and tightly adhered, is present all pipelines. Therefore, the potential for lift-off is ever present. There are two main assumptions in the use and acquisition of MFL inspection results. The first is that the sensors and magnetizer stay in close contact with the pipe wall. The second is that a "little debris won't affect MFL inspection results." Adherence to these assumptions invites the potential to underestimate or totally miss potentially injurious pipeline defects.

\subsubsection{SENSORS AND MAGNETIZER STAY IN CLOSE PROXIMITY OF THE PIPE WALL}

The assumption that sensors and magnetizers have maintained close contact with the pipe wall is widely held in the pipeline industry. Because the acquired MFL data is analyzed remote from the site in which the data is collected, the data analyst must assume that the data is free of the effects of debris unless the field inspection personnel provide information to indicate otherwise. Even when no visible signs of debris are present, it is still very possible 
that debris may have impacted the MFL inspection data. Conversely, if debris is observed to be present, there is no way to determine exactly where it affected the data and to what extent. Data analysis may provide indications of debris-induced lift-off, but these are not always reliable.

\subsection{2 “A LITTLE BIT OF DEBRIS WON'T AFFECT THE MAGNETIC FLUX LEAKAGE RESULTS."}

The misconception that "a little bit of debris won't affect the magnetic flux leakage results" is also widely held. The presence of debris may be determined either before or as a result of the MFL inspection. The effects of debris are often disregarded unless either the data contains signatures that indicate that significant sensor lift-off has occurred, the velocity profile is erratic, or investigative digs reveal significant discrepancies between the estimated and actual defect geometry.

This is why pipeline preparation is so important. It is not enough to inspect pipelinesthe pipelines must be properly prepared in order that the data obtained by the MFL inspection be as accurate as reasonably possible $[6,9,15,17]$. This assumption is prevalent because the impact of debris-induced lift-off on MFL results is not widely known or fully understood.

\subsection{PIPELINE CORROSION}

Corrosion is the degradation of a material as a result of reactions with its environment. The outcome is typically wall loss. If areas of localized wall loss go undetected they could potentially lead to catastrophic failure of the pipeline. Due to the wide variety of conditions on both the inside and outside surfaces of pipelines, corrosion cannot be totally eliminated [9]. However, it can be controlled and its progress monitored with the use of inline inspection tools.

Corrosion is more frequently found on the external surface of the pipeline because of the available electrolyte (soil) which is inherent of pipelines installed below grade [11]. Pipelines are typically installed with an external coating which isolates the pipe from the electrolyte (soil). However, breaks in the coating inevitably occur. These may be the result of material break down, soil stresses, or improper handling. These breaks expose the metal surface and may lead to the onset of corrosion (Figure 18).

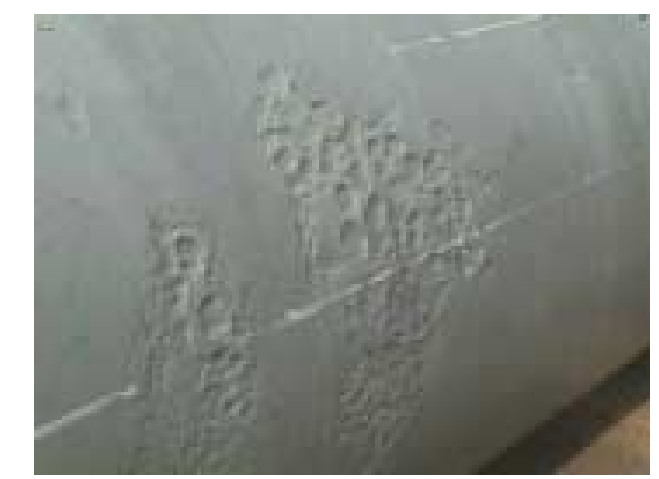

Figure 18 - External corrosion (Courtesy of GRI) [8]

Corrosion can also occur on the inside surface of the pipeline. Product quality is a significant factor in the control of internal corrosion. In the right conditions, constituents of the product itself can cause internal corrosion. Regular maintenance cleaning, the correct use 
of corrosion inhibitors and biocides, and maintenance of product quality are effective methods of controlling internal corrosion. Internal coatings are also available, but were not used on a wide scale on older pipelines. Figure 19 illustrates internal general corrosion with pitting contained within.

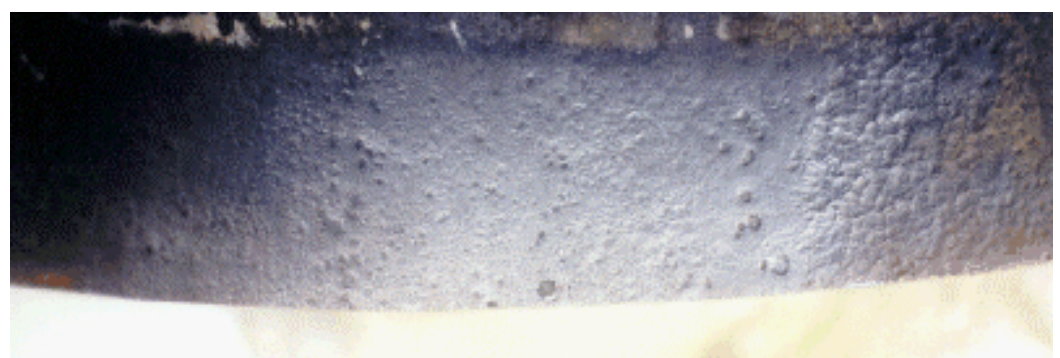

Figure 19 - Internal corrosion at 6 o'clock

\subsection{APPLICATION OF MAGNETIC FLUX LEAKAGE IN THE INSPECTION OF PIPELINES}

Inspection of underground pipelines is necessary to insure their safety and reliability. It would be impractical and uneconomical to excavate underground pipelines for inspection. Therefore, inspection tools utilizing a number of nondestructive testing technologies have been developed which travel through the pipeline between accessible locations along the pipeline such as compressor stations or valve settings. The inspection technology chosen depends on the goal of the inspection. Figure 20 depicts a high resolution MFL inspection tool which is commonly used to inspect pipelines.

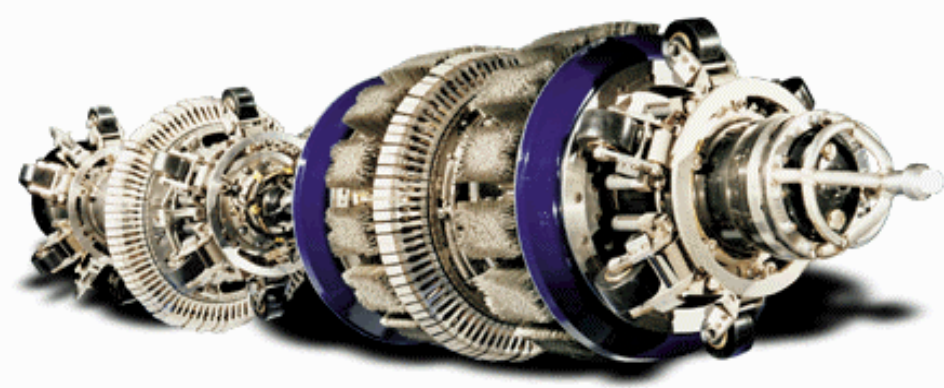

Figure 20 - High-resolution magnetic flux leakage inspection tool (Courtesy of PII) [18]

Other testing and monitoring data are used to supplement the MFL inspection results to determine the overall integrity of the pipeline [11]. These include close interval corrosion surveys, leakage surveys, facility patrols, product quality monitoring, coupon monitoring, geometry inspections, etc. These activities collectively form an overall integrity management program [11]. MFL inspection data is a major contributor to the integrity assessment process (Figure 21). 


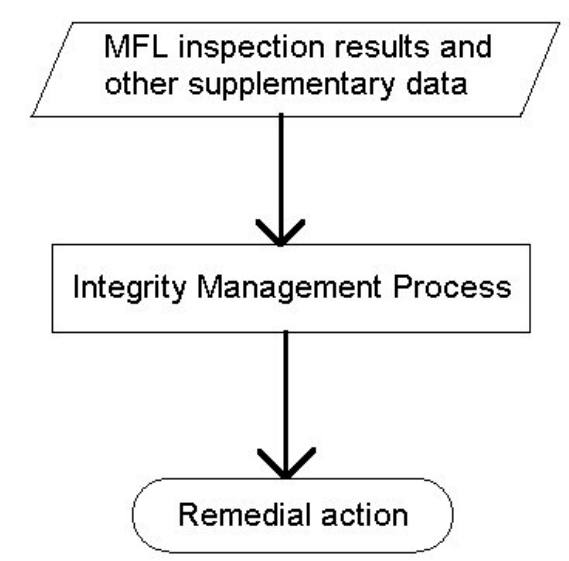

Figure 21 - Integrity management process

Pipelines are chosen for inspection based on a number of considerations. These include, but are not limited to, probability of failure, consequence of failure, age, operating history, gas quality, criticality to continuity of service, and population density. After the MFL inspection has taken place, inspection vendors provide a final report. The final report is a summary of the most severe anomalies that have been detected. The criteria typically used to produce an initial estimate of defect severity is B31G (based on depth and length estimation from MFL data). This criteria results in conservative estimates (compared to RSTRENG) of the remaining wall strength but is easy to calculate $[1,7]$.

Based on the calculated Maximum Safe Pressure (MSP), anomalies that threaten the current Maximum Allowable Operating Pressure (MAOP) are given first priority as operating pressure reductions are generally undesirable. The decision of whether or not further attention is given to an anomaly is largely based on this final report. Therefore, it is very important to ensure the accuracy of the acquired MFL data.

Some inspection vendors provide the raw data on compact disk with the associated data display software. This enables the pipeline operator to review the data themselves and keep for future reference. The software packages are typically proprietary and are not crosscompatible with other MFL inspection vendor's data. The data display software typically has two windows. One provides a visual representation of all of the data channels simultaneously (C-Scan in Figure 22). In this window the data is displayed as if the pipe were split axially and laid out flat on a table. The center is representative of the bottom of the pipe or the 6 o'clock position. The second window represents the data from a single channel (B-Scan of Figure 22). Cursors are available which can be used to measure between items or features on the display. 


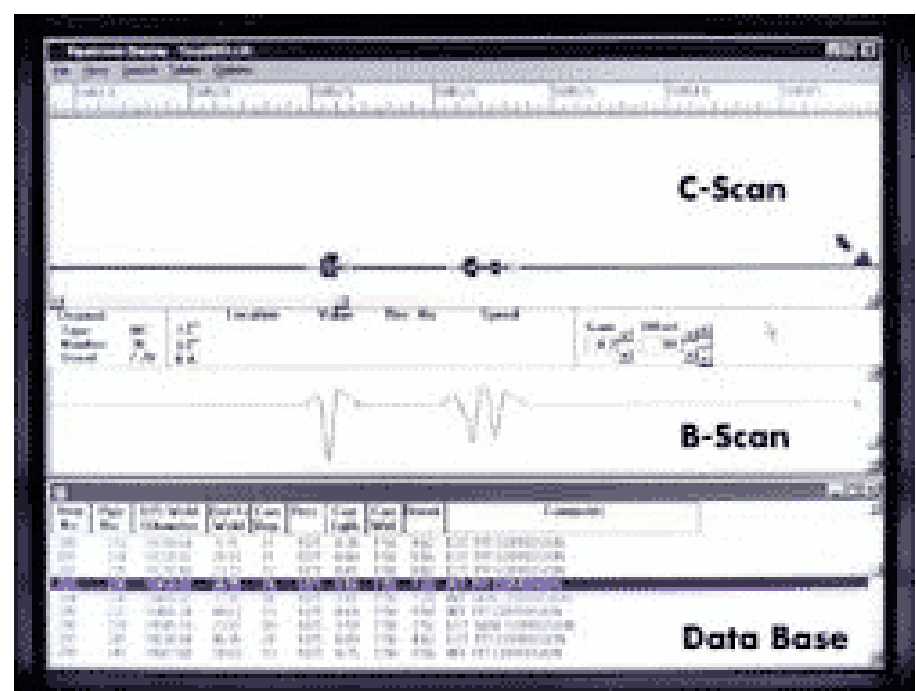

Figure 22 - Data display software (Courtesy of PII) [18]

When the operator has decided which anomalies it will investigate further, they are located with respect to the closest geographical reference point and marked for excavation. Once exposed, the anomalies are visually and nondestructively evaluated using ultrasonic thickness gauges, pit gauges, or laser profilometer. The actual wall thickness measurements obtained during this evaluation are then used to recalculate the MSP utilizing either the B31G or RSTRENG criterion. If the calculated MSP is not equal to or greater than the current MAOP of the pipeline, either the pipeline pressure is reduced to the calculated MSP or the corroded section of pipeline is removed and replaced with new pipe of suitable design.

From the forgoing discussion it is apparent that inline inspections are vital to pipeline operator's ability to maintain the integrity of their pipelines. MFL inspections help to prevent countless pipeline ruptures by providing a means to identify suspect pipeline before it fails catastrophically. However, this technology is not guaranteed to detect every defect even with the best of conditions and the presence of debris decreases the ability of the MFL inspection tool to accurately and reliably detect injurious defects. Therefore, more attention should be invested in the preparation of the pipeline for MFL inspection. 


\section{CHAPTER 3. DEBRIS-INDUCED LIFT-OFF}

\subsection{INTRODUCTION}

Debris-induced lift-off is the condition in which either the sensors, magnetizer, or both are separated from the pipe wall by pipeline debris $[8,17]$. Figure 23 depicts how debris is typically deposited in pipelines. All pipelines have the potential for debris accumulation which may be generated from a variety of sources. Debris may be in the form of paraffin, scale, compressor oil, black powder, salt, mineral buildup, rocks, hot tap coupons, welding slag, welding rods, dirt, dust, or various compositions of all of the above $[15,16,17]$. Debris can affect the MFL inspection tool's operation thereby affecting the accuracy of the inspection results $[6,8,9,13,15,17]$.

Figure 7 shows an example of a debris accumulation found in the bottom of a natural gas pipeline. The problem of lift-off is most frequently encountered at the bottom of the pipe between the 4 o'clock and 8 o'clock positions. Obscuring of the MFL signal in this region of the pipe is indicative of lift-off [17]. Debris accumulations are also common at low elevation points along a pipeline.

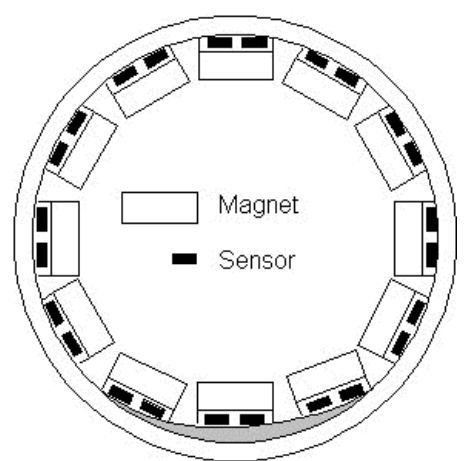

Figure 23 - Magnetizer and sensor lift-off by debris accumulation (end view) [17]

Debris-induce lift-off is an insidious problem because it is very difficult to detect [17]. Unless conspicuous signs are present to indicate that the acquired MFL data has been affected by debris, it is typically assumed that it was not [17]. If signs of debris are present, it is usually assumed that it was not enough to affect the MFL inspection results. In addition, there is no way to determine where and to what extent the debris-induced lift-off has affected the MFL data.

Ideally, the sensors and magnetizer should be in close contact with the pipe wall $[8,17]$. As the separation of the magnetizer and pipe wall increases, the level of flux transferred into the pipe wall decreases. Also, as the level of sensor lift-off increases, the measured peak flux leakage amplitude decreases $[2,8,15]$. Close contact of the sensors and magnetizers improves the detection and characterization accuracy of the detected anomalies $[2,8,13,17]$.

The quality of the inspection results is a function of pipeline cleanliness $[9,17]$. Industry experience has shown that even in small amounts, debris-induced lift-off can substantially degrade magnetic flux leakage inspection data [6]. Small amounts of loose debris can cause lift-off of the sensors. Large amounts of loose debris or tightly adhered 
deposits (paraffin, black powder, and salt) can cause lift-off of both sensor and magnetizer [17].

In practice, the two cases that typically occur are lift-off of either the sensors or of both the sensor and magnetizer simultaneously. Lift-off of the magnetizer alone could potentially occur on a bend, but the frequency in which lift-off of the magnetizer occurs is far less than that of sensor or simultaneous sensor and magnetizer lift-off.

\subsection{SENSOR LIFT-OFF}

The sensor elements are located at ends of sensors arms typically made of urethane (or other suitable material). The interference fit between the sensors and pipe and the resiliency of the sensor arm material is the only means of maintaining contact with the pipe wall. Therefore, sensors have much less resistance to debris-induced lift-off as compared to the magnetizer. Because if this lack of resistance, sensor lift-off is much more likely to occur than magnetizer lift-off in the presence of debris. Debris, both loose and tightly adhered can easily cause sensor lift-off. Therefore, if debris is present in the pipeline, it is unlikely that lift-off of the sensors can be avoided.

Sensor lift-off occurs naturally at tees, girth welds, wrinkle bends, or buckles [8]. These features can often be detected by observing the change in flux leakage signal magnitude. However, lift-off caused by debris is much more difficult to detect because no drastic changes in the flux leakage signal occurs [17]. With no definite, observable clues it is assumed that debris has not affected the flux leakage data [17].

To add to the complexity of the issue, detection of debris-induced sensor lift-off is elusive. Large levels of lift-off may be detected, but it is the smaller levels that present the greatest challenge. It affects the MFL signal, but leaves no trail or signature that it has occurred. At the present time, there is no way to detect low levels of sensor lift-off. As long as debris is present, sensor lift-off will always be a potential problem for MFL inspection of pipelines.

Boundary conditions exist at the interface between the pipe surface and air (Table 2) [8].

- The tangential component of the magnetic field $\left(\mathrm{H}_{\mathrm{T}}\right)$ is equal at the interface between the pipe and air.

- The normal component of the flux density $\left(\mathrm{B}_{\mathrm{N}}\right)$ is equal at the interface between the pipe and the air.

Table 2 - Boundary conditions at pipe surface [8]

In actuality, the sensors cannot be positioned in a manner which facilitates measurement of the flux leakage field at the pipe surface. Sensors are constructed with protective coatings to prevent damage which creates a base level of sensor lift-off. Therefore, the data that is collected has already experienced some initial decay, but this value is known (as opposed to debris-induced lift-off) and is consistent. When the sensor is separated from the pipe wall by an additional amount (by debris), the measured flux leakage magnitude is no longer representative of the actual value $[8,13,17]$.

The corrosion sensors are actually measuring the sum of two interacting magnetic fields. One is the flux leakage field generated by defects or other changes in magnetic 
properties and the other is an air(gas or liquid)-coupled field. These two fields coexist beyond the pipe surface and the sensor measures the combined effect of these two fields.

Figure 24 depicts the typical design of a sensor arm. The sensor element is mounted at the end of a fixed-length flexible arm which is attached to the magnet backing bar with threaded fasteners. The sensor arms are designed to keep the sensor head parallel to and in contact with the pipe wall. This ensures that the sensor element is in the proper orientation for the magnetic field component being measured.

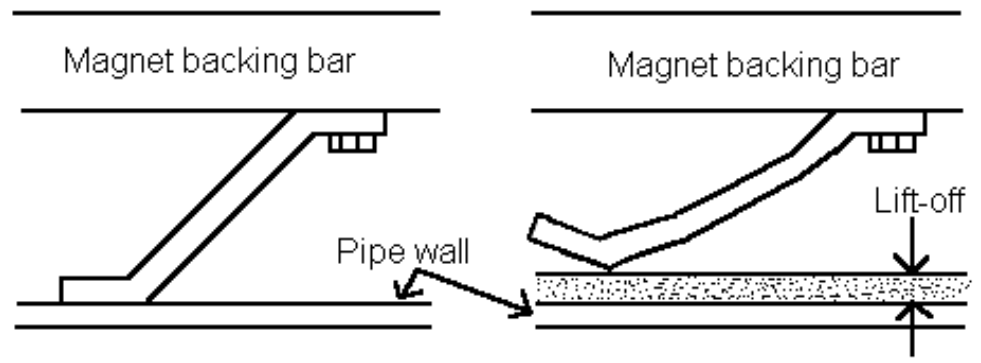

Figure 24 - Sensor head angle with/without debris-induced lift-off

When debris-induced sensor lift-off occurs, the angle between the sensor head and pipe wall increases. Due to the design of the sensor arm, the larger the magnitude of debrisinduced lift-off, the larger the resulting angle between the sensor element and the MFL field to be measured (Figure 25). As the angle between the sensor head and pipe increases, the magnitude of the measured MFL field decreases by $\cos (\alpha)$.

$$
\begin{aligned}
& H(\alpha)=H \cos (\alpha) \\
& \quad \text { Equation } 1
\end{aligned}
$$
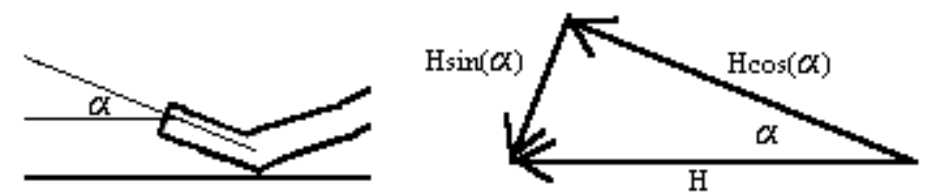

Figure 25 - Sensor head angle

As the sensor arm deflects to ride over debris, additional lift-off of the sensor element is generated. Suppose the sensor head is 0.75 inch in length and that the sensor element is positioned at the midpoint as shown in Figure 26. At an angular of 30 degrees, the additional lift-off is calculated as shown in Equation 2.

$$
L_{A}=\frac{0.75 i n c h}{2} \times \sin (30)=0.325 \text { inch }
$$

Equation 2

An additional of 0.325 inch of sensor lift-off $\left(\mathrm{L}_{\mathrm{A}}\right)$ is created in addition to the base debrisinduced lift-off. This further decreases the measured peak MFL amplitude. 


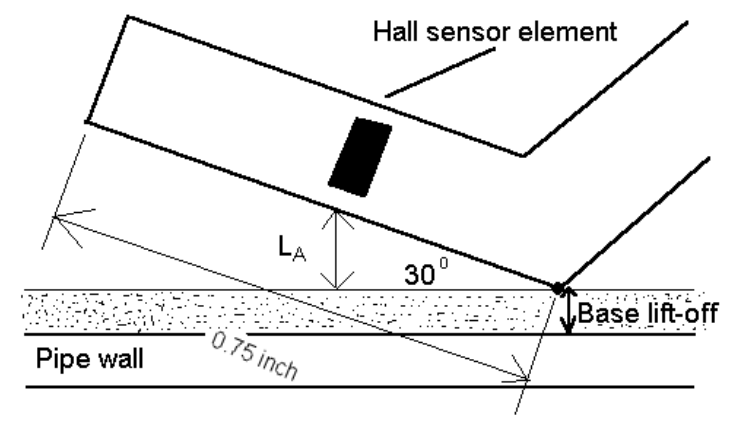

Figure 26 - Additional lift-off created by sensor head angle

The end result is an MFL peak reading that may be reduced for three reasons. First by virtue of the sensor head separation from the pipe wall, secondly by the cosine of the angle between sensor head and pipe wall, and thirdly by the additional lift-off created by the angular displacement of the sensor head.

It should also be noted that the angular displacement caused by debris-induced lift-off could potentially cause an overestimation of the peak MFL signal amplitude. On the leading side of the defect, the flux field lines are parallel or nearly parallel to the Hall sensor element (Figure 27). This results in an underestimation of the true magnitude of the tangential component of the leakage field at that location. On the leaving side of the defect, the sensor's alignment may be perpendicular or nearly perpendicular to the MFL field at this location. This can cause an overestimation of the tangential component of the MFL field which could lead to costly investigative digs to locate defects that may actually be benign.

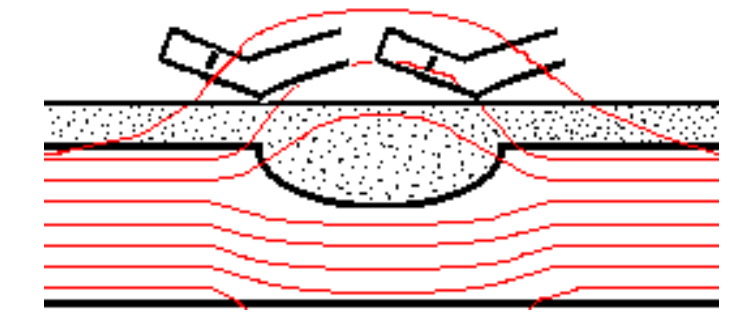

Figure 27 - Under and overestimation of field intensity

Lift-off of the ID/OD sensors decreases its sensitivity to changes in the magnetic field. If no change in signal change is detected by the ID/OD sensors, the flux leakage signal may be incorrectly interpreted. Large sums of money and time can be expended searching for anomalies that are thought to be on the outside of the pipe wall when they are actually on the inside $[8,17]$.

\subsection{MAGNETIZER LIFT-OFF}

Loose debris can potentially accumulate in amounts sufficient to cause the magnetizer to separate from the pipe wall surface. Layers of tightly adhered deposits can also cause separation of the magnetizer from the pipe wall. Lift-off of the magnetizer decreases the level of magnetization transferred into the pipe wall $[8,17]$. With lower levels of magnetic flux in the pipe wall, the level of flux leakage caused by defects is also reduced. In addition, if a magnetizer is providing a level of magnetization that is at or slightly above saturation, magnetizer lift-off can potentially cause the induced magnetization level to fall below 
saturation levels [8]. This could severely hinder the generation and thus the detection of flux leakage fields.

It is occasionally possible to identify magnetizer lift-off while analyzing the MFL data. Debris typically collects in the 4-8 o'clock position. Comparisons of the MFL signal at this location can be compared to other clock positions to determine whether magnetizer liftoff has occurred.

\subsection{SIMULTANEOUS SENSOR AND MAGNETIZER LIFT-OFF}

Accumulations of tightly adhered debris or large amounts of loose debris can cause lift-off of both the magnetizer and sensors simultaneously (Figure 28) [17]. This often occurs in the bottom half of the pipe. Defects under the influence of this type of lift-off have the greatest potential to go undetected or inaccurately sized.

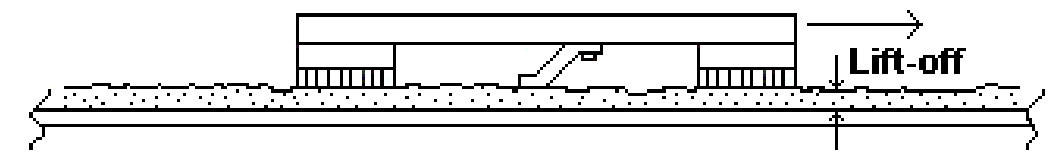

Figure 28 - Magnetizer and sensor lift-off by debris accumulation (side view)

\subsection{OTHER FACTORS THAT AFFECT MAGNETIC FLUX LEAKAGE DATA}

Besides the problem of lift-off there are a variety of other factors that can affect the MFL inspection tool's ability to detect and characterize corrosion anomalies. They include, but are not limited to tool velocity, stress effects, and remnant magnetization. However these are outside the scope of this research and will not be addressed. Reference 8 is an excellent source for additional information on these factors. 


\section{CHAPTER 4. METHODOLOGY}

\subsection{INTRODUCTION}

Test apparatus development and subsequent testing will attempt to qualitatively evaluate the effect of sensor, magnetizer and simultaneous sensor and magnetizer lift-off on the measured MFL signal. An MFL inspection tool records MFL magnitude versus axial length on a per channel basis. Multiple channels are arranged around the circumference of the MFL inspection tool to ensure adequate coverage around the pipe. However, for the purposes of this testing, it was only necessary to provide a single channel.

In a typical MFL inspection tool, the magnetizing assembly and sensors are mobile while the pipe is stationary. For simplicity and cost, it was much easier to design a test apparatus such that the magnetizer and sensor were stationary and the pipe coupon pulled axially across the magnetizer and sensor. This simulates the operation of a true MFL inspection device.

The apparatus designed to perform this testing is a simple and economical means of conducting in-house MFL testing and evaluation. It allows pipeline operators or researchers the ability to conduct independent MFL testing to qualitatively assess the impact of variables on the final flux leakage signal. It may also help the pipeline operator gain a better understanding of the MFL concept and how changes in variables affect the MFL signal.

\subsection{TESTING OBJECTIVES}

The objective of this testing was to qualitatively assess the impact of debris-induced lift-off on the acquired MFL data. Sensor, magnetizer, and simultaneous sensor and magnetizer lift-off will be examined and tests conducted to evaluate the effect of each on the measured MFL signal. The objectives for the testing are listed below.

1. Examine the effect of sensor, magnetizer, and simultaneous sensor and magnetizer lift-off on the measured MFL signal.

2. Compare the relative impact of sensor and magnetizer lift-off on the MFL signal to determine which has a greater effect on the measured MFL signal.

3. Determine what level of lift-off causes the greatest attenuation of the measured MFL signal.

\subsection{SENSOR LIFT-OFF}

Sensor lift-off is varied and the effect on the acquired MFL signal is observed for various types of artificial corrosion defects. To simulate various levels of sensor lift-off, the sensor's height relative to the pipe surface was varied by predetermined increments. The Hall sensor is rectangular in shape and is positioned at the end of the test probe. The tangential component of the flux leakage field was measured, so the Hall sensor tip was positioned perpendicular to the surface of the pipe and axially aligned

The smaller defects $(1,2)$ and the axially-aligned (5) defect required extra attention to ensure that the centerline (lateral or circumferential) of the defect is being examined. The lateral position of the sensor tip was adjusted laterally until the peak amplitude was achieved. The lateral position was then locked. Adjustments in the coupon guides were also made to 
prevent or remedy lateral movement of the pipe coupon. Once the lateral position of the coupon/defect was set, the vertical position of the sensor tip was then set and locked.

\subsection{MAGNETIZER LIFT-OFF}

For each corrosion defect, the magnetizer lift-off was varied by predetermined increments. Lift-off of the magnetizer was achieved by placing 2 spacers on each magnet pole. This produces a consistent lift-off of the pipe coupon from the magnet brush surface.

\subsection{SIMULTANEOUS SENSOR AND MAGNETIZER LIFT-OFF}

By using the lift-off methods described in the forgoing discussion, various combinations of sensor and magnetizer lift-off were examined. By varying the amount of lift-off of both the sensor and magnetizer, it was possible to assess the relative impact of sensor, magnetizer, and simultaneous sensor and magnetizer lift-off on the acquired MFL signal.

\subsection{TESTING PROCEDURE}

To facilitate accurate, repeatable results, the following test procedure was developed and the steps are outlined on Table 3.

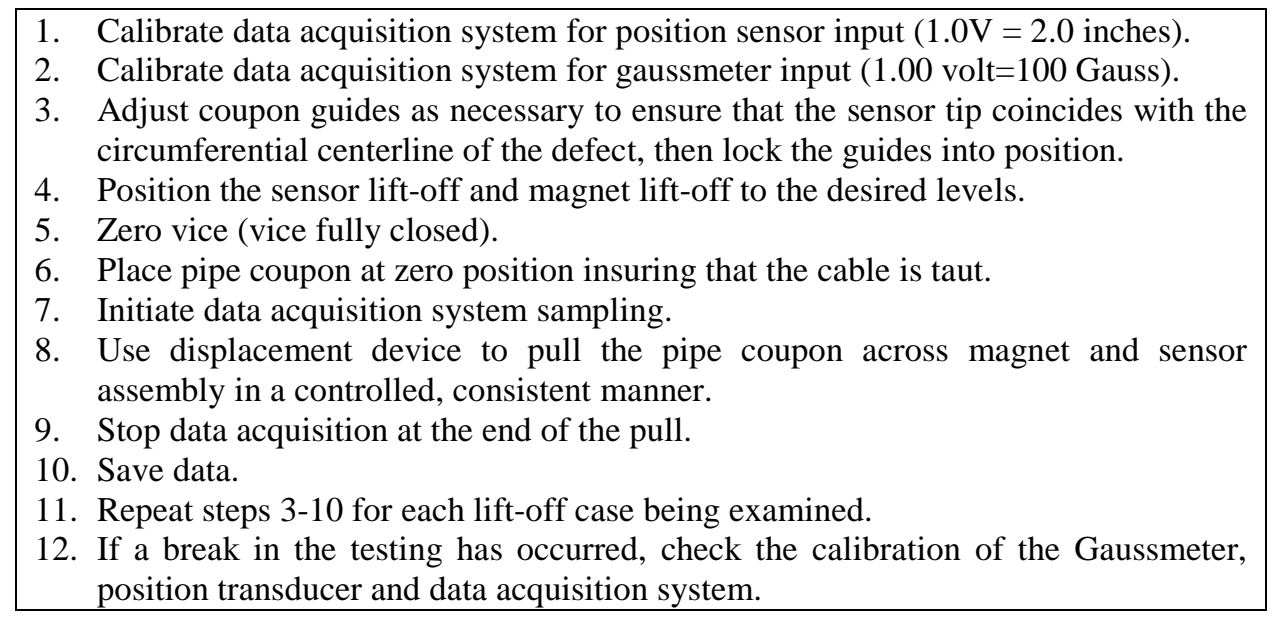

Table 3 - Test procedure

After calibration, voltage readings corresponding to the actual limits of the corrosion anomalies were recorded. These readings are provided in the Table 4. These values are subject to error as the beginning and end of the defects were visually estimated. Even so, the values are very close the actual dimensions.

\begin{tabular}{|c|c|c|c|c|c|}
\hline Anomaly & $\begin{array}{c}\text { Start } \\
\text { Voltage (V) }\end{array}$ & $\begin{array}{c}\text { End } \\
\text { Voltage (V) }\end{array}$ & $\begin{array}{c}\text { Start Distance } \\
\text { (inch) }\end{array}$ & $\begin{array}{c}\text { End Distance } \\
\text { (inch) }\end{array}$ & $\begin{array}{c}\text { Estimated } \\
\text { Distance (inch) }\end{array}$ \\
\hline 1 & 2.665 & 2.913 & 5.33 & 5.826 & 0.496 \\
\hline 2 & 2.476 & 2.717 & 4.952 & 5.434 & 0.482 \\
\hline 3 & 2.417 & 3.181 & 4.834 & 6.362 & 1.528 \\
\hline 4 & 2.228 & 2.985 & 4.456 & 5.97 & 1.514 \\
\hline 5 & 2.326 & 3.273 & 4.652 & 6.546 & 1.894 \\
\hline 6 & 2.554 & 2.633 & 5.108 & 5.266 & 0.158 \\
\hline
\end{tabular}

Table 4 - Voltages corresponding to anomaly axial length 
These values were not included as part of the graphs because the data was primarily concerned with the degradation in the peak and baseline amplitudes of the MFL signal.

The testing procedure generated many data files. To organize these files, a system was developed in which the file name indicated the test case performed. A prefix was used to represent each defect, magnetizer lift-off case, and sensor lift-off case. The anomalies had a prefix of A. The magnetizer lift-off had an M prefix. The sensor lift-off had an L prefix. Table 5 summarizes the organization prefixes of the file names.

\begin{tabular}{|c|c|c|c|c|c|}
\hline Defect & $\begin{array}{c}\text { Defect } \\
\text { code }\end{array}$ & $\begin{array}{c}\text { Magnetizer lift-off } \\
\text { magnitude (inch) }\end{array}$ & $\begin{array}{c}\text { Magnetizer lift- } \\
\text { off code }\end{array}$ & $\begin{array}{c}\text { Sensor lift-off } \\
\text { magnitude } \\
\text { (inch) }\end{array}$ & $\begin{array}{c}\text { Sensor lift-off } \\
\text { code }\end{array}$ \\
\hline 1 & A1 & 0 & M0 & 0 & L0 \\
\hline 2 & A2 & 0.125 & M1 & 0.125 & L1 \\
\hline 3 & A3 & 0.25 & M2 & 0.25 & L2 \\
\hline 4 & A4 & 0.5 & M3 & 0.5 & L3 \\
\hline 5 & A5 & 0.75 & M4 & 0.75 & L4 \\
\hline 6 & A6 & 1.0 & M5 & 1.0 & L5 \\
\hline
\end{tabular}

Table 5 - File name codes

For example, for the case involving defect 1 at zero sensor lift-off and zero magnetizer liftoff the file name is A1L0M0.WQD. 


\section{CHAPTER 5. TEST APPARATUS DESIGN}

\subsection{PRIMARY TEST APPARATUS COMPONENTS}

The test apparatus must be able to measure and record magnetic flux leakage (Gauss) and displacement (inches). This testing is accomplished with the use of the primary components outlined in Table 6. Specifications for these components are provided in Appendices 1-4.

\begin{tabular}{|l|l|}
\hline \multicolumn{1}{|c|}{ Primary Components } & \multicolumn{1}{c|}{ Purpose } \\
\hline Position Transducer & Measure displacement \\
\hline Gaussmeter & Measure flux leakage field magnitude \\
\hline Magnet & Magnetize the pipe \\
\hline Data Acquisition System & Collect and store acquired data \\
\hline \multicolumn{2}{|c|}{ Table 6 - Primary Components of test apparatus } \\
\hline
\end{tabular}

Figure 29 illustrates how these components are interconnected for the test procedure.

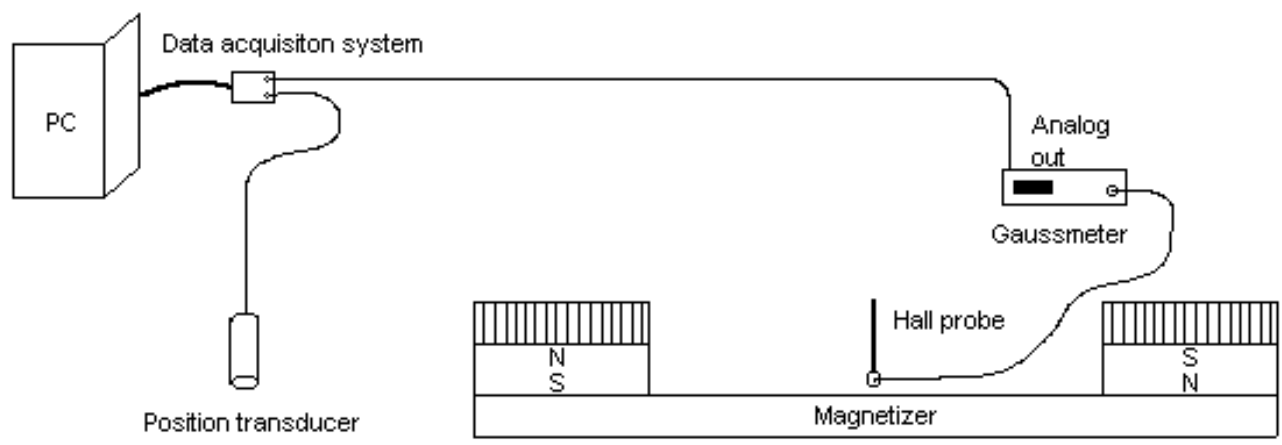

Figure 29 - Layout of primary components

\subsubsection{POSITION TRANSDUCER}

The position transducer (PT) is manufactured by SpaceAge Controls, Inc. The particular model used was a 160 series PT and hae the capability to accurately measure up to 16 inches of travel. With a Tenma laboratory power supply, voltage is applied to the proper terminals of the PT. An output voltage was measured across the appropriate terminals which was proportional to the length of cable drawn from the PT housing. Depending on the method in which the terminals are connected, the change in output voltage can either increase or decrease with displacement. 


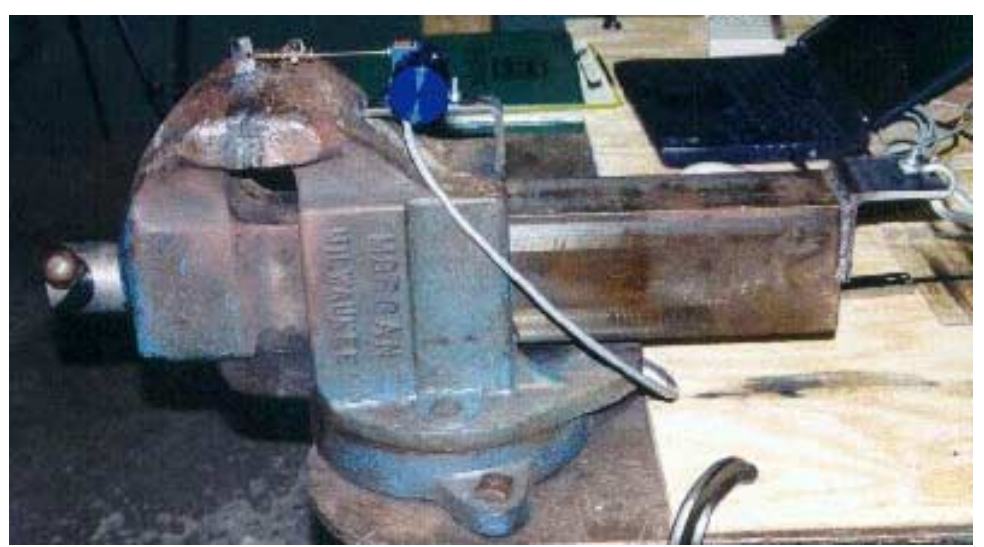

Figure 30 - Position transducer mounted to vice (side view)

The PT was mounted to brackets that were welded to the body of a bench-top vice (Figure 30). The vice had a maximum stroke of approximately 10.25 inch. For each test, approximately 10 inch of stroke was recorded. The PT was mounted to the stationary jaw of the vice and the end of the PT cable was mounted to the mobile jaw of the vice as shown in Figures $30 \& 31$. This arrangement was chosen because this would minimize the error produced in the measurement of pipe coupon displacement.

Once this PT is positioned, it would be out of the way of the rest of the test apparatus. This strategy precluded the use of attachments to the pipe coupons which would have required changing of the PT cable mounting with each defect and each coupon. More detailed information on this device is located in the Appendix 8.

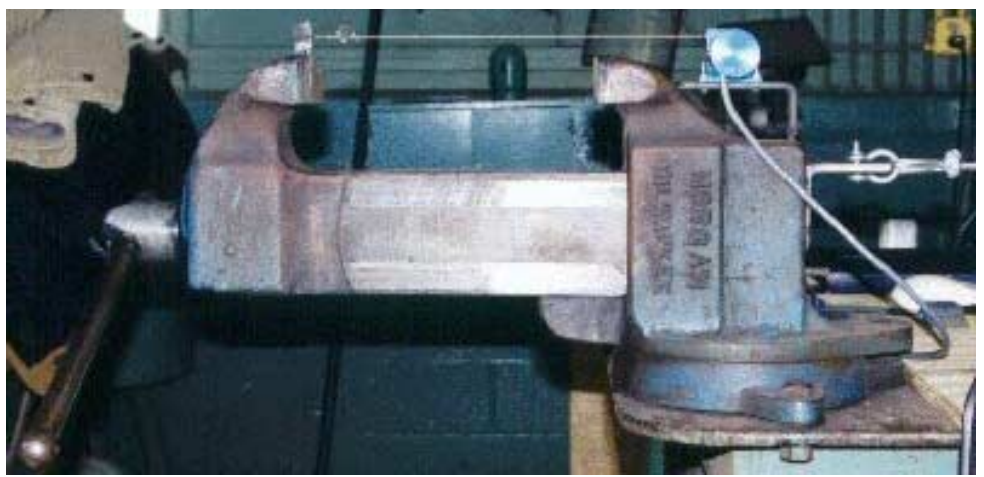

Figure 31 - Position transducer cable fully extended

As the jaws of the vice were opened, it pulled the pipe coupon across the magnetizer via the steel cable and shackles. At the same time, cable is being drawn from the spool within the PT. This causes a change in the output voltage of the PT which is proportional to the jaw's displacement. Therefore, displacement is determined by relating a voltage change in the PT to the actual displacement.

The PT is factory calibrated so that $1.0 \mathrm{~V}$ is approximately equal to 1 inch. Recall that the PT is designed to measure up to 16 inches of travel. If the PT were supplied with 16 $\mathrm{V}$, a displacement of 1 inch would be equivalent to approximately 1 Volt. However, $16 \mathrm{~V}$ is beyond the input voltage range of the data acquisition system $( \pm 10 \mathrm{~V})$. Therefore, approximately $8.3 \mathrm{~V}$ was supplied to the PT. 


\subsubsection{GAUSSMETER}

The gaussmeter is manufactured by Walker Scientific, Inc. (Figure 32). The gaussmeter consists of a meter box, a Hall sensor probe, and a wiring harness which connects the two. This device measures the flux leakage field magnitude in Gauss. A transverse probe is positioned in a manner that facilitates the measurement of the tangential component of the magnetic flux leakage field.

This particular model provides an analog output which is proportional to the measured MFL field magnitude. For the reading shown (143.3 Gauss) in Figure 32 the analog output reading would be $1.433 \mathrm{~V}$. The analog out signal provides the input for one channel of the data acquisition system. The 100 Gauss range was used for the testing because it provided the best resolution for the range of flux leakage levels examined. More detailed information on this device is located in the Appendix 2.

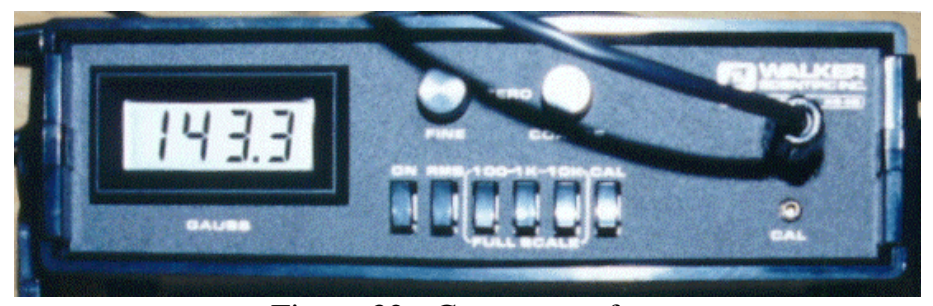

Figure 32 - Gaussmeter face

\subsubsection{MAGNET}

The magnet used in this testing procedure was originally used in a 24 inch MFL tool (Figures 33, 34, 35). It was borrowed from the GRI Pipeline Simulation Facility in Columbus, $\mathrm{OH}$. This one magnet section weighs approximately $80 \mathrm{lbs}$. The magnet is made of neodymium-iron-boron, has a maximum energy product of 28 megagauss-oersted, and a total area of 18 in $^{2}$ (3in. $x$ 6in).

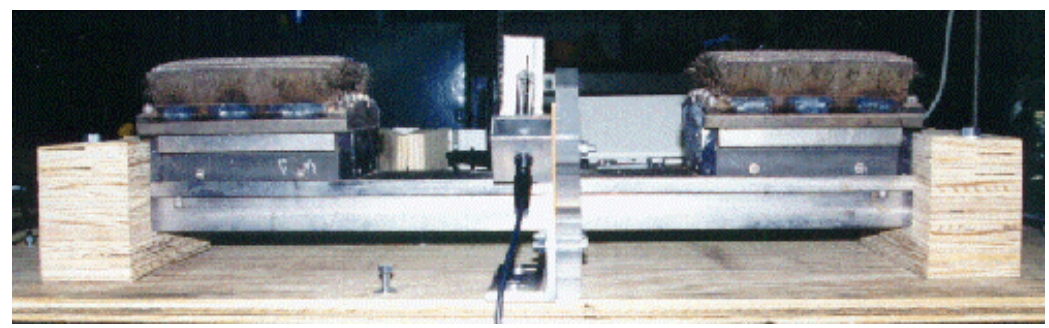

Figure 33 - Magnetizing assembly (side view)

It was necessary to elevate the magnetizer so that there would be ample room to adjust the height of the sensor tip. This was accomplished with blocks constructed of multiple layers of plywood sheets (Figures 33, 34, 35). The 2 blocks were then bolted to the table with $3 / 8 \times 5-1 / 2$ inch carriage bolts to prevent lateral, axial, and vertical movement. 


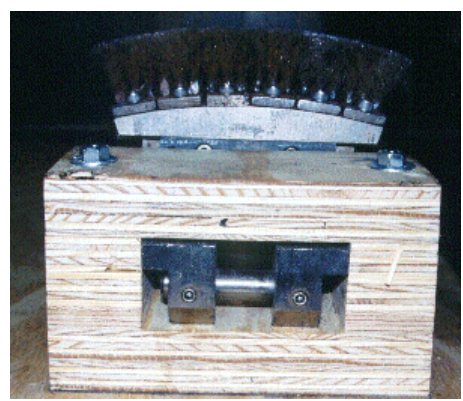

Figure 34 - Magnetizing assembly (end view)

The sensor was located between the magnet poles and below the pipe coupon (Figures 33, 35). As the pipe coupon is pulled axially across the magnet, the sensor at its prescribed level of lift-off measured the flux leakage field generated by the defect.

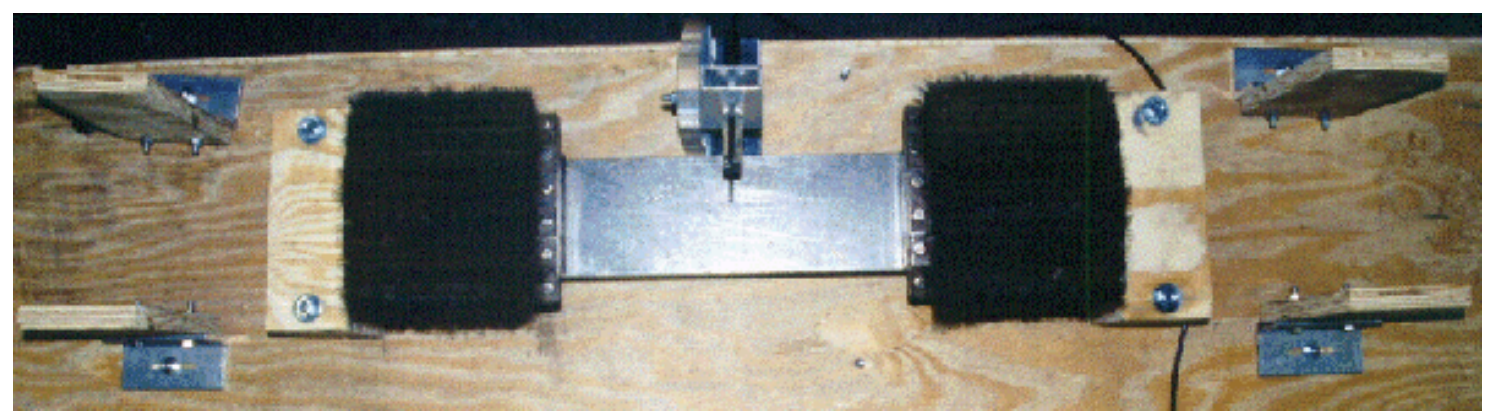

Figure 35 - Magnetizing assembly (top view)

\subsubsection{DATA ACQUISITION SYSTEM}

The data acquisition system used for this testing procedure was the DI-151RS manufactured by DATAQ. This 12 bit system has two input channels and has a maximum sampling rate of $240 \mathrm{~Hz}$. It conveniently connects to the serial port (RS-232) of any personal computer for portability and compatibility.

Channel 1 receives its input from the analog out signal produced by the gaussmeter which is representative of magnetic flux leakage magnitude. Channel 2 receives its signal from the PT which represents displacement. The data acquisition system and its input devices can be viewed in Figure 36. More detailed information on the data acquisition system is located in Appendix 4. 


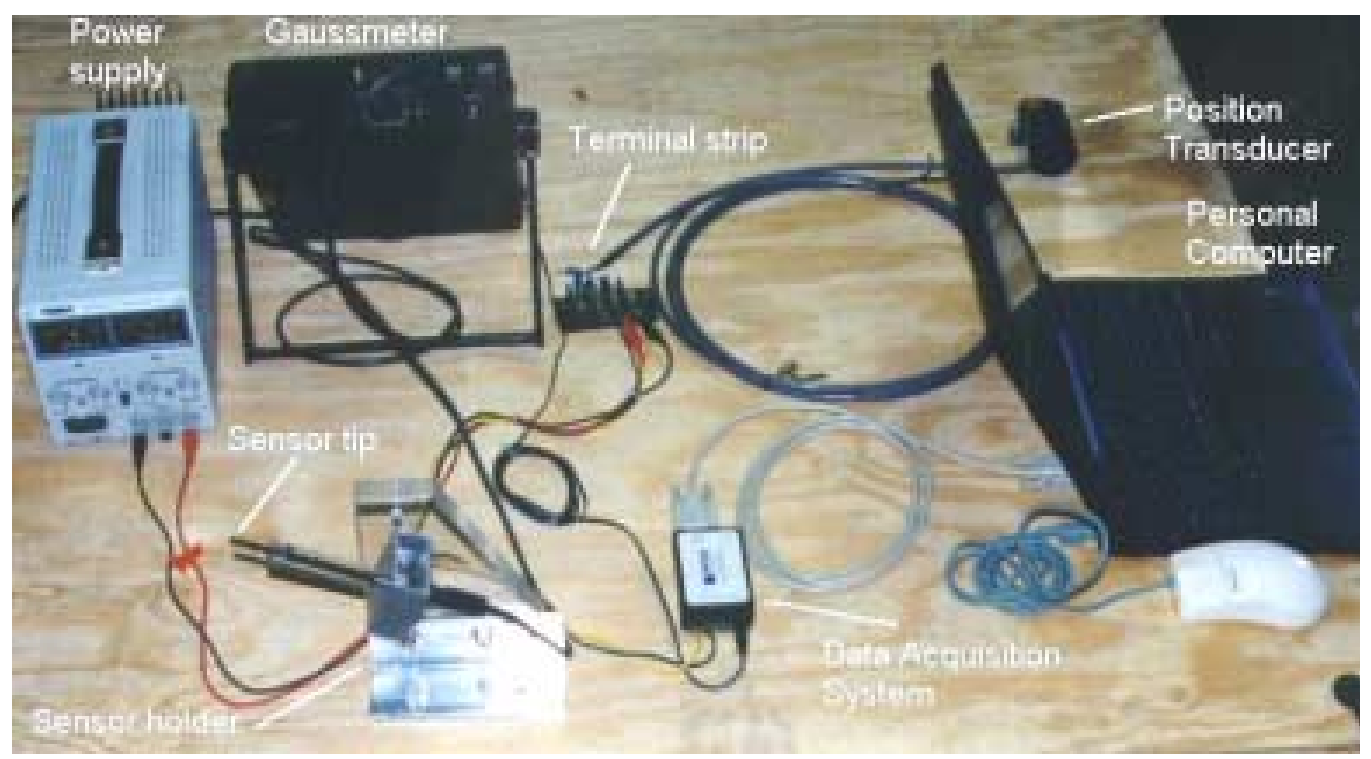

Figure 36 - Data acquisition system and inputs

To keep the file sizes at a manageable level a sampling rate of $120 \mathrm{~Hz}$ was prescribed. Therefore, 60 samples per second were recorded for each channel. This sampling rate produced approximately 1500 data points in a span of 10 inches. The DATAQ software interfaces with the data acquisition module and allows the data to be saved to a file name with ${ }^{*}$.WQD extension. Figure 37 illustrates the software screen display.

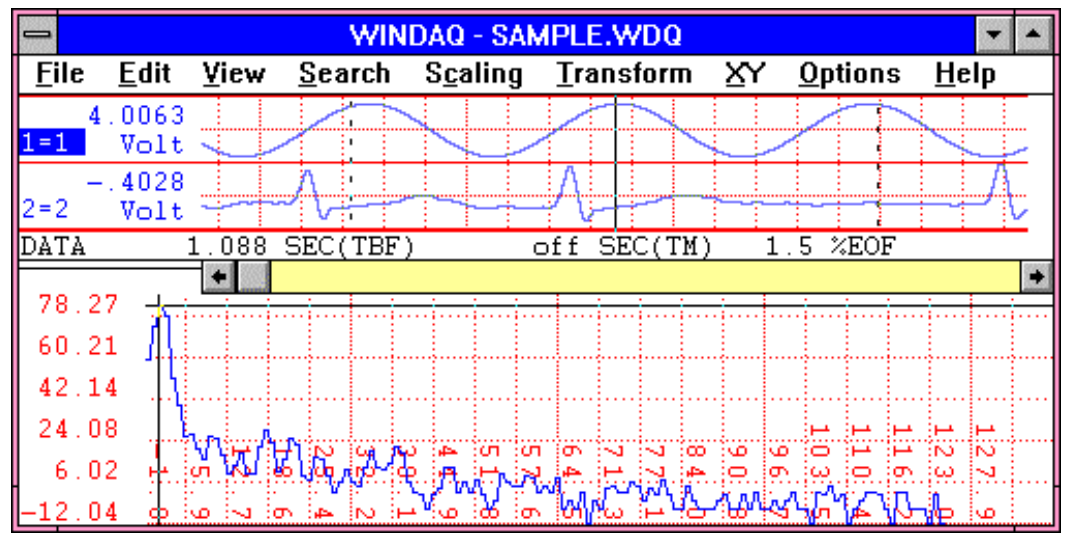

Figure 37 - WINDAQ software interface [19]

The software package is then used to export the data to a *.DAT file which can be read by common spreadsheet programs. This file was accessed with a spreadsheet program and the data copied and pasted to a new spreadsheet that contains all sets of data for the given test case. When all the data sets were acquired and transferred to appropriate spreadsheet, they can be displayed graphically for review, analysis, and post-processing.

\subsection{SECONDARY TEST APPARATUS COMPONENTS}

Additional components were necessary to facilitate successful testing and data acquisition. These secondary components are summarized in Table 7. 


\begin{tabular}{|l|l|}
\hline Secondary Components & Purpose \\
\hline Hall sensor holder & $\begin{array}{l}\text { Hold sensor tip at the desired position (various levels of sensor } \\
\text { lift-off) }\end{array}$ \\
\hline Pipe coupons & Provide the medium for flux leakage testing \\
\hline Coupon guides & Ensure that the coupon does not experience lateral movement \\
\hline Displacement device (Vice and cable) & $\begin{array}{l}\text { Pull the coupon across the magnetizer in a controlled fashion for } \\
\text { up to 10 inches }\end{array}$ \\
\hline Magnet spacers & Simulate various levels of magnetizer lift-off \\
\hline
\end{tabular}

\subsubsection{HALL SENSOR HOLDER}

Sensor lift-off was to be simulated by this test apparatus. To accomplish this, a method of varying the sensor tip lift-off of the sensor tip from the pipe surface at predetermined increments $(0,0.125,0.25,0.5,0.75$, and 1 inch $)$ was required. In addition, the device should allow an adjustment of the lateral position of the sensor tip in the event of variations from coupon to coupon. The device must also have the capability of locking its position once it has been set.

A device was designed and fabricated that provided all of the forgoing requirements (Figure 39). The device was constructed of $2 \times 2 \times 0.25$ inch aluminum angle, $2 \times 1 \times 0.125$ inch aluminum tubing, 0.625 inch polycarbonate tubing, and 0.75 inch thick acrylic (Plexiglass). These materials (non ferrous) were chosen to minimize/eliminate their influence on the flux leakage fields. The Hall sensor probe is delicate, so it was placed inside the 4 inch long section of polycarbonate tubing and the annulus filled with clear silicone. This allowed the sensor to be rigidly mounted with damaging the sensor probe itself and improved repeatability in the lift-off simulation.

The sensor is positioned midway between the magnet poles and does not change once set. The sensor holder has two slots inside which 0.25 inch bolts may translate. One slot is oriented laterally (or circumferentially) and the other is oriented radially. This provides the ability to make lateral and radial adjustments of the sensor tip position. The bolts may be tightened to lock the sensor tip into position. Details on the design of this device are in Appendix 6.

It was not possible to position the Hall sensor probe perpendicular to the pipe surface because of space restrictions, so an alternate position was determined. To ensure that the readings were consistent from test to test, the sensor angle relative to the pipe surface was maintained at 45 degrees (Figure 38).

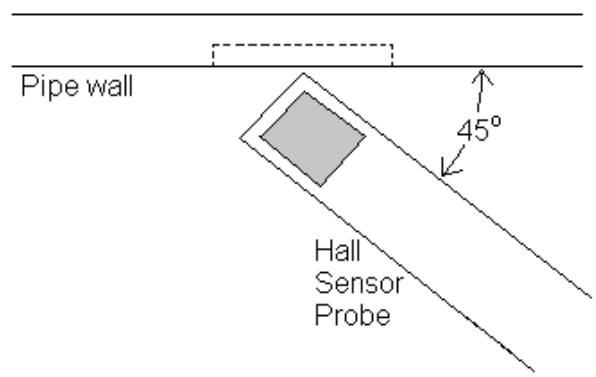

Figure 38- Sensor tip at 45 degree angle 
The design of the sensor holder allowed the position of the sensor tip to varied laterally and vertically while maintaining the prescribed 45 degree angle with the use of a 45 degree triangle as shown in Figure 39.

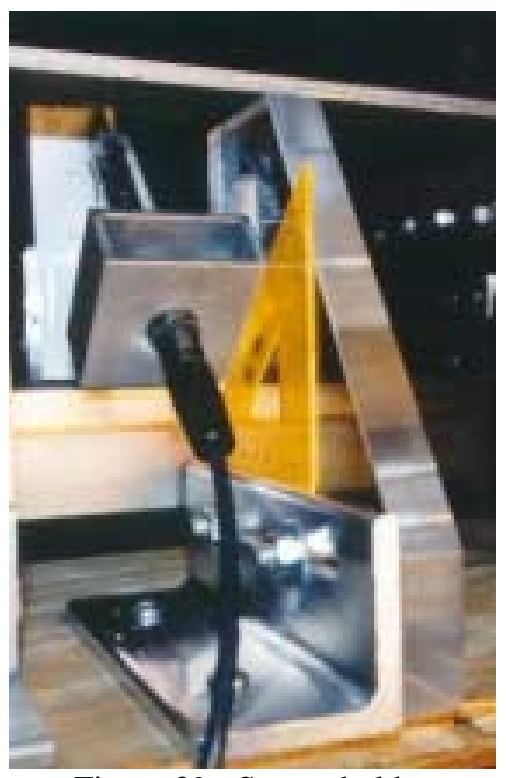

Figure 39 - Sensor holder

\subsubsection{PIPE COUPONS}

Pipelines are predominantly constructed of steel. To ensure strong leakage fields and realizing that the magnet will not have the benefit of adjacent magnetizers, a small wall thickness was chosen. Therefore, the coupons were constructed of nominal 0.25 inch A36 plate steel cut to 56-1/2 inch in length and 7 inch in width (Figure 40). Ultrasonic measurements of the steel's thickness measure $0.223 \pm 0.003$ inch.

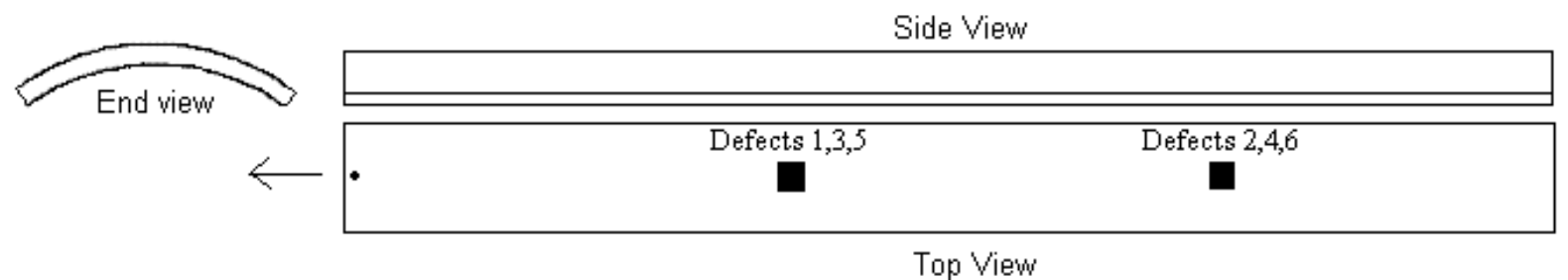

Figure 40 - Pipe coupon

Three coupons were fabricated and each contains two defects. The defects are spaced 18 inch apart to minimize flux leakage field interaction. At one end of the coupon is a 0.375 inch hole which serves as a point at which the shackle can be secured. Having only one point to pull the pipe coupon also ensures that the coupon is magnetized in the same direction for each test.

The defects were machined into the pipe coupon with a milling machine. The flat steel coupon, was then bent at $\sim 0.375$ inch increments to a 12 inch radius relative to the outside surface. A 12 inch radius in the pipe coupon was necessary so that its profile matched the profile of the brush on the magnetizer. The coupons were constructed in this manner because it is much easier to machine artificial corrosion defects into a flat rather than 
a curved piece of steel (Figure 41). Details on the design of the pipe coupons are available in Appendix 5.

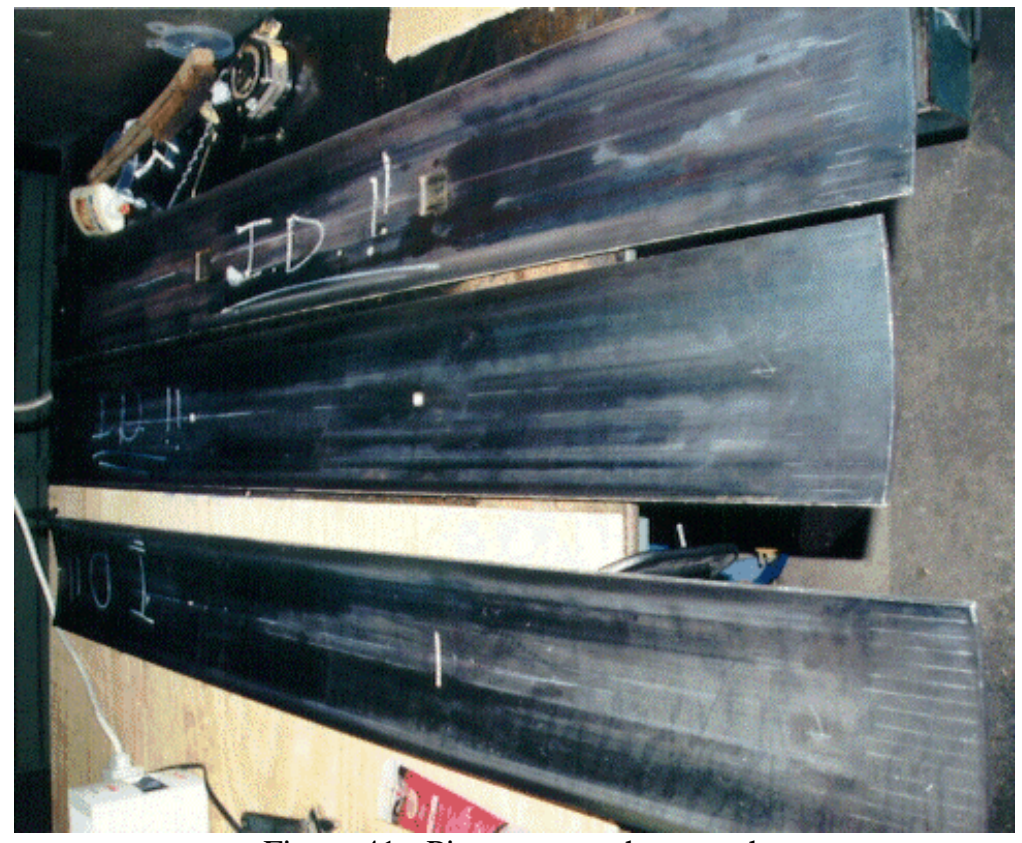

Figure 41 - Pipe coupon photograph

\subsubsection{PIPE COUPON GUIDES}

To prevent lateral movement of the pipe coupons as they are pulled across the magnet, coupons guides were designed and fabricated (Figure 35, 42). These guides were constructed of $2 \times 2 \times 0.25$ inch aluminum angle and $4 \times 9$ inch pieces of 0.75 inch plywood. The bases of the coupon guides are slotted to allow its position to be adjusted.

Once the position was set, a test pull of the coupon commences to ensure that the circumferential centerline of the pipe coupon coincides with the sensor tip. If any lateral drift occurred, the guides were reset and re-tested. Design details on the design of the coupon guides are provided in Appendix 7. 


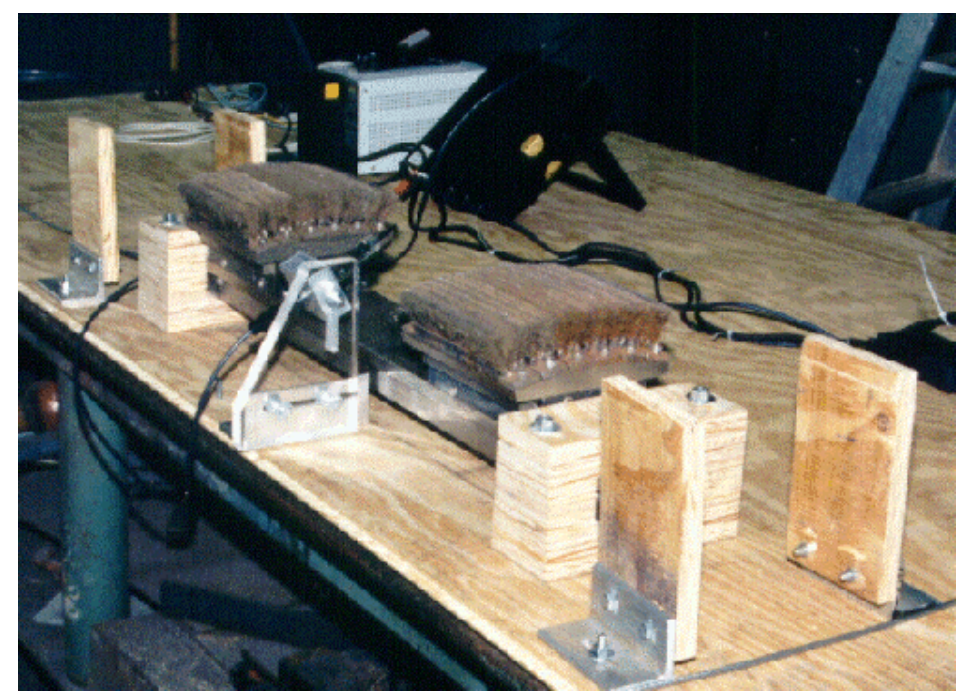

Figure 42 - Coupon guides (end view)

\subsubsection{DISPLACEMENT DEVICE}

The force required to pull the pipe coupon across the magnet is estimated at $50 \mathrm{lbs}$. For data acquisition purposes, a slow, straight, repeatable pull is required. This was provided by a bench top vice. A short piece of $3 \times 3 \times 0.25$ inch steel angle was welded to the end of the movable section of the vice. Figure $30 \& 31$ provides views of this device. Appendix 8 contains design details of this device and the PT mounting brackets.

Cable and shackles are used to connect the vice and pipe coupon. The cable size and strength were chosen to minimize elastic deformation. The cables used in the testing procedure were $0.25 \mathrm{inch}$ and had a working strength of $1400 \mathrm{lbs}$. The force subjected to these cables was 2 orders of magnitude below the working strength of the steel cable. Therefore, elastic deformation was not considered a factor.

Because there are two defects per pipe coupon and the distance between the magnet and displacement device was fixed, two cables with different lengths were required. The first was 24-3/16 inch in length and the second was 42-11/16 inch in length. The long cable was used for defects 1,3,5 while the short cable used for defects 2,4,6. Using these standard cable lengths ensured that the start point of each defect was consistent and repeatable from test to test.

\subsubsection{MAGNET SPACERS}

To simulate the effect of magnetizer lift-off, magnet spacers were designed and constructed (Figure 43). The levels of magnetizer lift-off were predetermined increments of $0.125,0.25,0.5,0.75$, and 1 inch. Lift-off of the magnetizer should be consistent and repeatable. The spacers should also have good wear characteristics to stand up to the friction between it and the pipe coupon surface. All these qualification were satisfied by magnet spacers constructed of clear acrylic (Plexiglass).

Four spacers for each magnetizer lift-off increment were constructed. Two were used on each magnet pole to separate the pipe coupon surface from the magnet brush surface. Tabs were bonded to the end of the spacers to prevent axial translation of the spacer when the pipe coupon is being pulled across the magnet. Design details are provided in Appendix 8. 

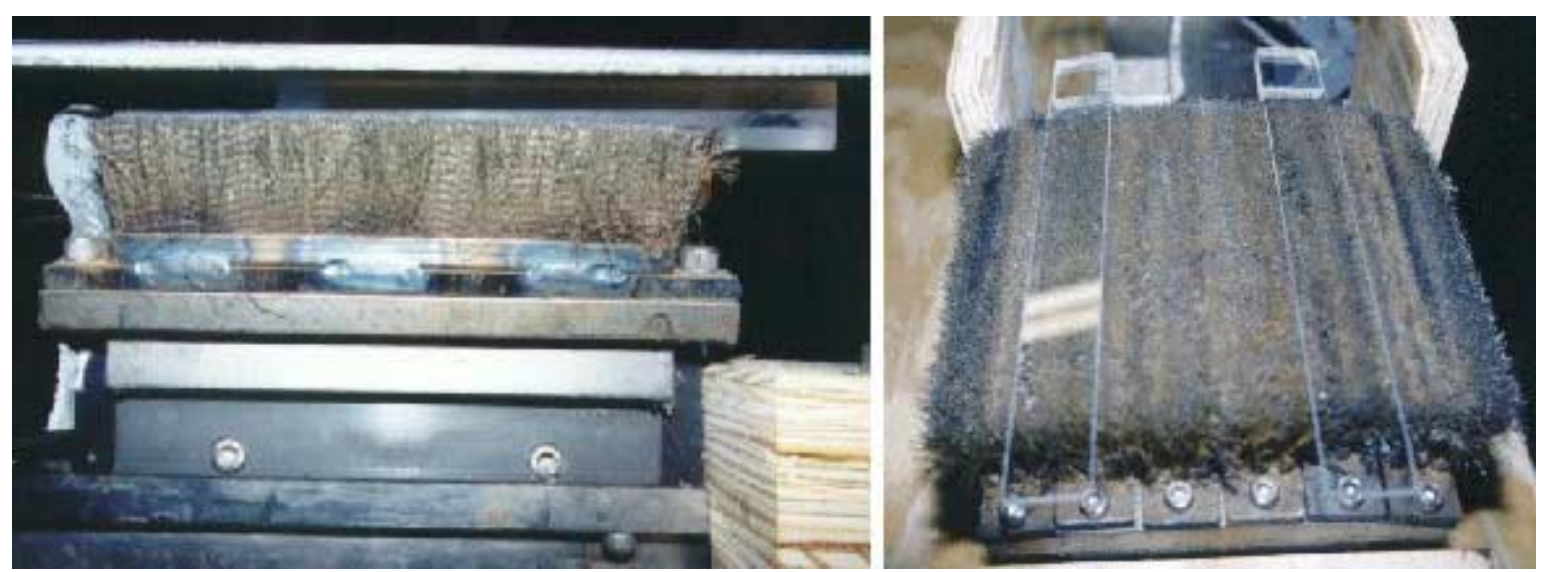

Figure 43 - Magnet spacing devices (side and top view)

\subsection{DEFECT SET}

In the inspection of pipelines there are some basic types of corrosion that can be encountered. These include general corrosion, shallow pitting, deep pitting, axially-oriented slot corrosion, and circumferentially-oriented slot corrosion. A set of 6 defects were machined into steel coupons and their dimensions are summarized in Table 8. Testing of these various defects should provide some insight into the effect of lift-off on various defect geometries. Two corrosion defects per coupon were machined into what would be representative of the inside surface of the pipe.

\begin{tabular}{|l|l|l|l|l|l|}
\hline Defect & $\begin{array}{c}\text { Length } \\
\text { (inch) }\end{array}$ & Width (inch) & $\begin{array}{l}\text { Wall thickness } \\
\text { (inch) }\end{array}$ & $\begin{array}{l}\text { Remaining Wall } \\
\text { thickness (inch) }\end{array}$ & \% Wall Loss \\
\hline 1 & 0.5 & 0.5 & 0.243 & 0.164 & 32.5 \\
\hline 2 & 0.5 & 0.5 & 0.244 & 0.083 & 66.0 \\
\hline 3 & 1.5 & 1.5 & 0.243 & 0.158 & 35.0 \\
\hline 4 & 1.5 & 1.5 & 0.242 & 0.088 & 63.6 \\
\hline 5 & 1.875 & 0.375 & 0.245 & 0.168 & 31.4 \\
\hline 6 & 0.375 & 1.875 & 0.244 & 0.164 & 32.8 \\
\hline
\end{tabular}

Table 8 - Defect set dimensions 


\subsection{TEST APPARATUS OVERVIEW}

Figure 44 provides an annotated photo to illustrate the test apparatus configuration.

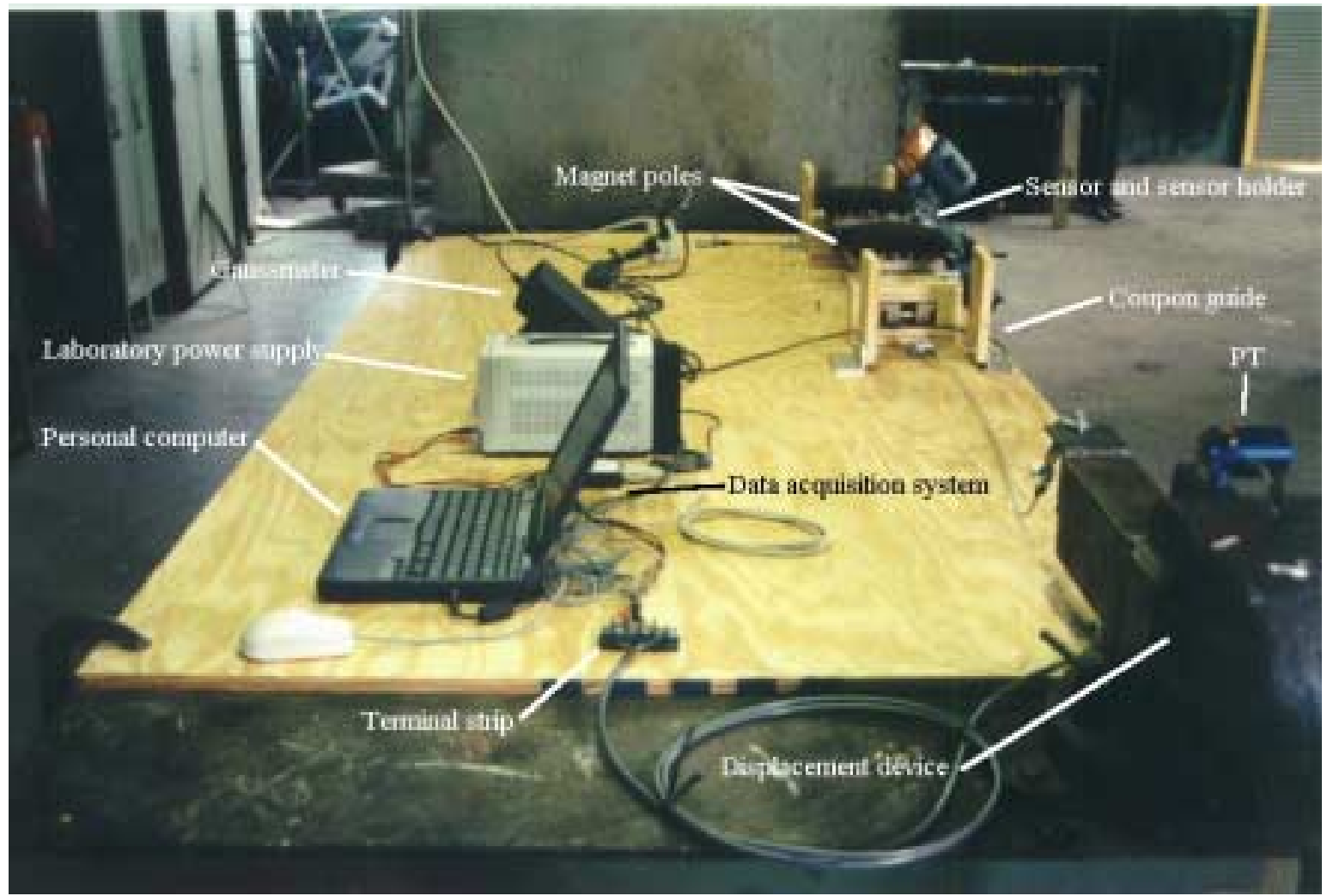

Figure 44 - Test apparatus overview 


\section{CHAPTER 6. ERROR ANALYSIS}

\subsection{INTRODUCTION}

It would be impossible to produce a quantitative estimate of the degradation in the MFL signal that would be applicable to all MFL testing procedures. There are many variables that can affect the acquired MFL data and many of these variables are not consistent with every test procedure. Some of these include magnet strength, sensor types, data acquisition system capabilities, metal properties and geometries, and defect geometry, to name a few. Thus, the findings of this test procedure are only valid for this specific test apparatus.

Therefore, the goal of this testing was to qualitatively assess the impact of magnetizer and/or sensor lift-off on MFL inspection results. Two distinct quantities are measured during the test procedure. They are magnetic flux leakage magnitude and distance in Gauss and inches, respectively. Once the data sets are acquired they are plotted against one another. Each of these quantities is subject to error which will now be examined.

\subsection{DISPLACEMENT MEASUREMENT ERROR}

The data acquisition system is calibrated so that a change of 1.0 Volt is proportional to a displacement of 2.0 inches. The manufacturer's data indicates a linear measurement error of $\pm 0.35 \%$ of the range for which the PT was designed. The PT had a 16 inch range. Therefore, the PT data is accurate to \pm 0.056 inch $(0.0035 \times 16$ inch $)$. Recalling the calibration of 1 volt to 2 inches of actual displacement, the error in the final displacement measurement is \pm 0.112 inch $(2 \times 0.056$ inch).

Elastic deformation of the cables were examined as a possible source of error. The largest force is required at the start of the pull. Once the displacement device has overcome the static friction, the force required to pull the pipe coupon is substantially reduced and is consistent for the length of the test pull. Therefore, elastic strain of the cable was not considered as a source of displacement error. Any additional errors in the distance measurement were systematic.

\subsection{MAGNETIC FLUX LEAKAGE MEASUREMENT ERROR}

Error associated with the measurement of flux leakage magnitude is of primary concern because the results of this testing procedure are used to assess the effect of debrisinduced lift-off on the measured flux leakage magnitude. Review of the testing procedure indicates that the measurement of flux leakage amplitude is subject to additional sources of error.

There is some error to consider in the gaussmeter itself. The gaussmeter is factory calibrated to provide, at a minimum, the specifications contained in Appendix 2. The 100 Gauss range was selected for this experiment. The specifications for the gaussmeter state a $0.05 \%$ of full scale error. In the 100 Gauss range, the error is then \pm 0.05 Gauss (100 Gauss $x$ 0.0005). This error is relatively small. However, the error in the probe must also be considered. The probe specifications convey a $1 \%$ linearity of reading up to 20 KGauss. The probe, therefore, has an error of 10 Gauss in the 1000 Gauss range (1000 Gauss X 0.01) and an error of 1 Gauss in the 100 Gauss range (100 Gauss X 0.01). 
Therefore, the probe is the dominant factor with regards to MFL measurement error. The maximum measured MFL signal amplitude for this testing was 373 Gauss, so the maximum probe error due to linearity of reading was 3.73 Gauss (373 Gauss x 0.01). Therefore, as a worst case estimate, the error due to the combined effect of gaussmeter and probe linearity is estimated at 3.78 Gauss $(3.73+0.05$ Gauss $)$.

Sensor tip position error is clearly evident in the test procedure. This error is due, primarily, to the fact that the sensor's tip position (lift-off) must be visually estimated (Figure 45). This introduces a certain amount of error. The level of lift-off is visually estimated and is accurate to approximately $\pm 1 / 32$ inch to $\pm 1 / 64$ inch. This estimation can introduce error into the flux leakage measurement. Review of the data suggests that this error has its greatest impact on the acquired MFL signal at very low levels of sensor lift-off (from 0 to 0.125 inch). As the magnitude of lift-off increases the effects of sensor tip position error diminishes.

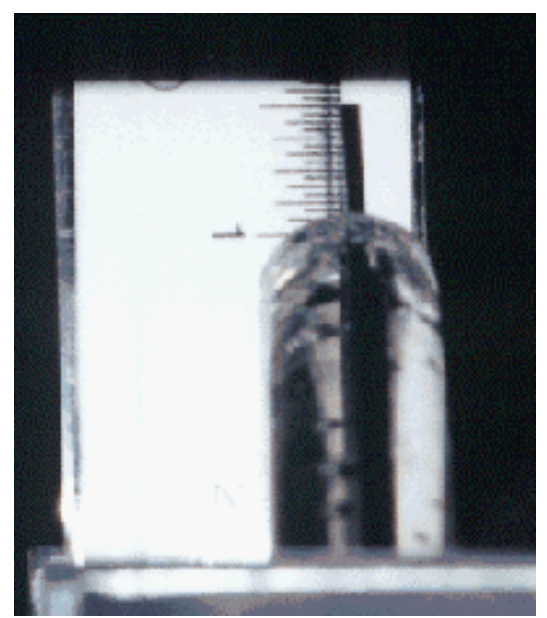

Figure 45 - Probe tip lift-off measurement of 0.5 inch

Defect 1 data (Appendix 11) for the case of zero magnetizer lift-off will be used as a basis for estimating the magnitude of sensor tip position error. At zero sensor lift-off, the maximum measured flux leakage is 156 Gauss and at 0.125 inch lift-off, 122 Gauss is measured. Hence, in a span of 0.125 inch the measured peak MFL amplitude reduced by 34 Gauss. Assuming a linear relationship between lift-off and flux leakage reduction, a 1/32 inch error in lift-off would result in an error of approximately 8.5 Gauss (34 Gauss/4). However, this error would only be at the maximum at very low levels of lift-off.

\subsection{DATA ACQUISITION SYSTEM ERROR}

When recording data with a data acquisition system it is desirable that the data acquisition system to produce a quantization error that is less than the error of the variables being sampled [14]. Variations in the steel's properties can generate noise or error in the signal on the order of a 2-3 Gauss [8]. It has been established that the gaussmeter produces an error of 3.78 Gauss. Therefore, the data acquisition system must be able to convert the analog data to digital data while keeping the quantization error below the level error of its inputs signals.

A 12 bit data acquisition system was used for this testing procedure. Therefore, it had a maximum resolution of $4096\left(2^{12}\right)$ counts across the $20 \mathrm{~V}( \pm 10 \mathrm{~V})$ maximum input range. 


$$
\begin{aligned}
& 20 \mathrm{~V}=4096 \text { counts } \\
& \text { Equation } 3
\end{aligned}
$$

Dividing both sides by 20 yields Equation 4.

$$
\begin{aligned}
& 1.0 \mathrm{~V}=204.8 \text { counts } \\
& \text { Equation } 4
\end{aligned}
$$

Therefore, the data acquisition is able to resolve a change of $1 \mathrm{~V}$ into 204.8 increments or counts. The gaussmeter analog output is factory calibrated so that $1 \mathrm{~V}$ is equal to 100 Gauss in the 100 Gauss range setting.

$$
\begin{aligned}
& 1.0 \mathrm{~V}=100 \text { Gauss } \\
& \text { Equation } 5
\end{aligned}
$$

By equating Equations 4 and 5 yields Equation 6.

$$
\begin{aligned}
& 204.8 \text { counts }=100 \text { Gauss } \\
& \text { Equation } 6
\end{aligned}
$$

Dividing both sides of Equation 7 by 204.8 yields the quantization error associated with the MFL measurement.

$$
\begin{gathered}
1 \text { count }=0.488 \text { Gauss } \\
\text { Equation } 7
\end{gathered}
$$

Therefore, the data acquisition system is able to resolve the flux leakage measurement data into 0.5 Gauss which is well below the error generated by the Gaussmeter (3.73 Gauss), sensor tip position error (8.5 Gauss), or variations in the steel's magnetic properties (2-3 Gauss). Hence the data acquisition system is sufficient resolution to preclude the generation of additional error in the analog to digital conversion of MFL input data.

The distance measurement is now considered. The PT, when supplied an input voltage, produces an output voltage that is proportional to the amount of spool rotation. The data acquisition system was calibrated so that a change in 1.0 Volts is proportional to a change 2 inches.

$$
\begin{gathered}
1.0 \mathrm{~V}=2.0 \text { inches } \\
\text { Equation } 8
\end{gathered}
$$

By equating Equations 4 and 8 yields Equation 9.

$$
204.8 \text { counts }=2.0 \text { inch }
$$

Equation 9

Dividing both sides of Equation 9 by 204.8 yields the quantization error associated with the displacement measurement.

$$
\begin{gathered}
1 \text { count }=0.00977 \text { inch } \\
\text { Equation } 10
\end{gathered}
$$

The data acquisition system is able to resolve the displacement data to 0.01 inch which is slightly below the error generated by the PT itself $( \pm 0.112$ inch). Therefore, the data 
acquisition system has sufficient resolution to preclude the generation of additional error in the analog to digital conversion of displacement data.

\subsection{ERROR SUMMARY}

In summary, the errors of each measured variable are within tolerable limits and are not of a magnitude that would invalidate the results of this research. More accurate test components could have been used, but the qualitative results would have remained the same. The position data is considered to be reasonably accurate $( \pm 0.112$ inch or $0.7 \%$ full scale) for the purposes of this test procedure. The flux leakage measurements, on the other hand, are subject to additional error because of sensor tip position error. Summing the errors in the measurement of the magnetic flux results in a total error of \pm 12.28 Gauss $(8.5$ Gauss +3.78 Gauss) or $\pm 6.14 \%$ of full scale (200 Gauss). This is, of course, a worst case estimate of MFL error. Graphing MFL versus displacement data and considering the error estimates yields Figure 46.

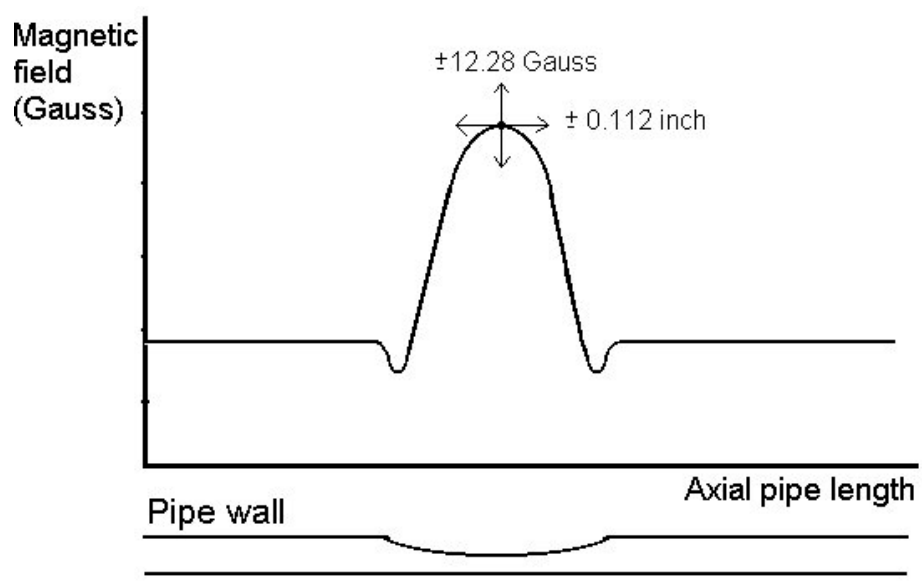

Figure 46 - Flux leakage and distance measurement error

In practice, the maximum error in each test component will not be experienced [14]. Errors may be positive as well as negative. When a test is conducted with a number of components, a composite error may be calculated. When the individual accuracies are independent of one another, the Root Mean Square (RMS) method of error estimation may be used. This is also called the probable systematic error [12].

This error is calculated by taking the square root of the sum of the squares of the individual errors as shown in Equation 13. $\mathrm{E}_{1}$ is representative of the sensor probe linearity error and $E_{2}$ is representative of the sensor tip position error. The gaussmeter error has been omitted for this calculation because of its extremely small value $(0.05$ Gauss $)$ in comparison with the probe linearity and gaussmeter error.

$$
E_{1}=\left(\frac{3.73}{200}\right)=0.01865
$$




$$
\begin{gathered}
E_{2}=\left(\frac{8.5}{200}\right)=0.0425 \\
\quad \text { Equation } 12 \\
E_{R M S}=\sqrt{\sum_{1}^{N} E^{2}}=\sqrt{0.01865^{2}+0.0425^{2}}=0.0464 \\
\text { Equation } 13
\end{gathered}
$$

In summary, the RMS error calculated indicates an error in the MFL reading of $\pm 4.64 \%$ or \pm 9.28 Gauss. The RMS error calculation results in a more realistic estimate of error. The previous estimate of \pm 12.29 gauss. In either case, the levels of error were not of a magnitude which would have caused the results and conclusions to be considered suspect. Revising Figure 46 to reflect the RMS error yields Figure 47

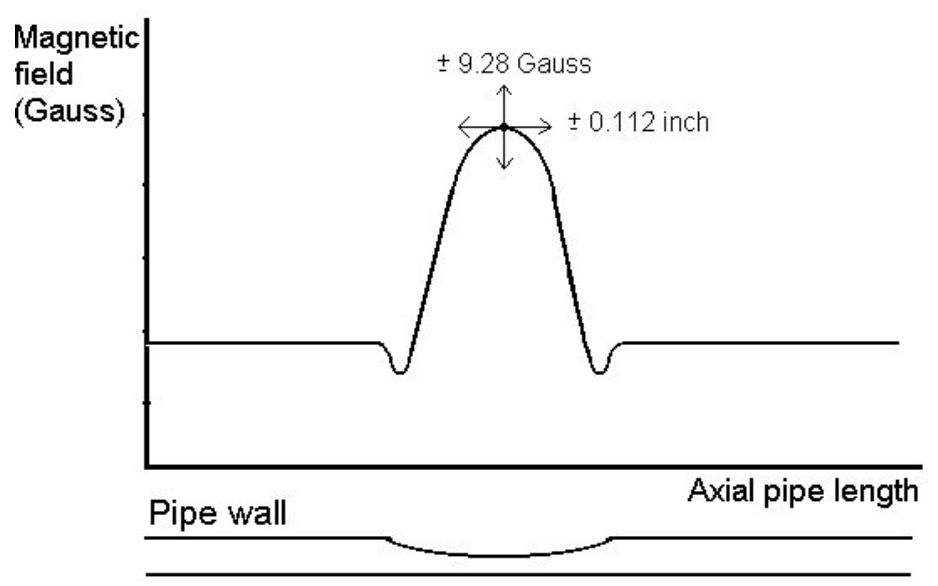

Figure 47 - RMS error. 


\section{CHAPTER 7. RESULTS}

\subsection{RESULTS}

As previously stated, the results and conclusions drawn from this testing procedure are qualitative in nature. There are a multitude of factors that influence the MFL signal and to repeat every one of them would be extremely difficult. This is why the quantitative results generated by this test procedure cannot be applied to all MFL testing. However, qualitative results should be consistent with all MFL testing procedures and should provide valuable insight into the effects of debris-induced lift-off on MFL testing.

The detrimental effects of lift-off are clearly evident in all MFL tests conducted. The results of this research strongly suggest that debris-induced lift-off, in all its forms (sensor, magnetizer, or both), has a negative impact on MFL data. In all cases examined, lift-off caused inaccurate measurement of the true magnitude of MFL field. Analysis of this inaccurate MFL data could lead to defects either being missed or undersized. This may have a significant impact on the integrity of the pipeline system.

Listed below are the most significant results of this testing procedure.

1. Lift-off (sensor, magnetizer, and both simultaneously) causes a reduction in the measured peak and baseline magnetic flux leakage amplitudes for all anomaly types.

2. Lift-off of sensors and magnet simultaneously produces the greatest reduction in the measured peak magnetic flux leakage amplitude for all anomaly types.

3. The geometry of the defect has an impact on the amount of signal attenuation is produced by lift-off.

4. Sensor lift-off has a substantially greater effect on the peak magnetic flux leakage amplitude than magnetizer lift-off of the same magnitude.

5. Low levels of sensor lift-off have a significant affect on the measured peak flux leakage magnitude.

\subsubsection{LIFT-OFF (SENSOR, MAGNETIZER, AND BOTH) CAUSES A REDUCTION IN BOTH THE MEASURED PEAK AND BASELINE MAGNETIC FLUX LEAKAGE AMPLITUDE FOR ALL DEFECT TYPES.}

Appendix 20 summarizes the results of Appendix 11-13. Appendix 20 illustrates the effect that lift-off has on the measured peak MFL amplitude. These graphs clearly show the reduction in measured peak amplitude with increasing lift-off for all cases of lift-off examined. In addition, it is evident that the geometry of the defect has an effect on the attenuation of the measured peak amplitudes.

Appendix 22 aids in the illustration of baseline depression as a result of lift-off. The data points used in Appendix 22 were also derived from the graphs of Appendix 11-13 at a common location ( 0.999 Volt $=\sim 2$ inches) to provide a basis of comparison. These graphs indicate that all defect types experience a depression of the baseline magnetic flux leakage amplitude with increasing lift-off.

The final analysis of acquired MFL data is largely based on comparisons of measured peak amplitudes to measured baseline amplitudes. If either is affected, interpretations of the MFL data may be inaccurate. Appendix 20 shows that significant reductions in the measured peak MFL amplitudes can result from sensor and simultaneous sensor and magnetizer liftoff. In practice, lift-off of the magnetizer alone occurs least often. Lift-off is most likely to 
be either by sensor or simultaneous sensor and magnetizer lift-off. It is also these cases that produce the highest level of signal degradation due to lift-off.

\subsubsection{LIFT-OFF OF SENSORS AND MAGNET SIMULTANEOUSLY PRODUCES THE GREATEST REDUCTION IN MEASURED PEAK MAGNETIC FLUX LEAKAGE AMPLITUDE FOR ALL DEFECT TYPES EXAMINED.}

Appendices 14-19 compare the zero lift-off case (no sensor or magnetizer lift-off) to the sensor, magnetizer, and simultaneous sensor and magnetizer lift-off cases at each level of lift-off. This provides an indication of the relative impact on the measured peak magnetic flux leakage amplitude. This comparison shows that the case of simultaneous sensor and magnetizer lift-off case produces the greatest reduction in the measured peak MFL amplitude. It was also interesting to note that magnetizer lift-off produced the greatest reduction in measured baseline MFL amplitude.

\subsubsection{DEFECT GEOMETRY HAS AN INFLUENCE ON THE AMOUNT OF REDUCTION IN MEASURED PEAK MAGNETIC FLUX LEAKAGE AMPLITUDE PRODUCED BY LIFT-OFF.}

Appendix 20 and 21 provide insight into the effect that defect geometry has on the amount of reduction in measured peak MFL amplitude with increasing lift-off. Appendix 20 shows that deeper and wider anomalies produce the highest flux leakage amplitudes at zero lift-off. This is because they produce the largest obstruction to axial magnetic flux travel within the pipe wall. By examining the change in MFL peak amplitude versus displacement for each increment of lift-off it is also apparent that the deeper and wider defects have larger slopes. This suggests that the reduction in measured peak flux leakage amplitude due to liftoff is greater for deeper and wider defects.

Appendix 21 also indicates that as the width (circumferentially) and depth of a defect increases, so does the amount of reduction in the measured peak MFL amplitudes. The data points used in Appendix 21 are derived from the peak MFL amplitudes in Appendix 20. They are calculated by taking the difference in the readings between each increment of liftoff and dividing by the peak MFL amplitude (for the zero lift-off case). This value is then multiplied by 100 . For defect 2 , the data point for the first increment of lift-off $(0-0.125$ inch) is calculated as shown in Equation 14.

$$
\% \operatorname{Re} \text { duction }=\frac{(216-158)}{216} \times 100=26.9 \%
$$

\subsubsection{SENSOR LIFT-OFF HAS A SUBSTANTIALLY GREATER EFFECT ON THE PEAK MAGNETIC FLUX LEAKAGE AMPLITUDE THAN MAGNETIZER LIFT-OFF OF THE SAME MAGNITUDE.}

Appendices 14-19 compare the no lift-off case to sensor, magnetizer, and simultaneous sensor and magnetizer lift-off cases at each level of lift-off. This provides an indication of the relative impact reduction in the peak magnetic flux leakage amplitude for the various levels of lift-off. The data indicates that sensor lift-off produces a much greater 
reduction in measured peak magnetic flux leakage amplitude than magnetizer lift-off of the same magnitude.

These graphs also show that for most cases, sensor lift-off produces nearly the same reduction in measured peak flux leakage amplitude as simultaneous sensor and magnetizer lift-off. This suggests that when simultaneous sensor and magnetizer lift-off occurs, the majority of the signal attenuation is due to the lift-off of the sensors. Also evident is the indication that magnetizer lift-off has the greatest influence on the baseline flux leakage level while sensor lift-off has the greatest influence on the measured peak flux leakage amplitude.

\subsubsection{LOW LEVELS OF SENSOR LIFT-OFF HAVE A SIGNIFICANT EFFECT ON THE MEASURED PEAK FLUX LEAKAGE AMPLITUDE.}

Appendix 21 assists in determination of the level of lift-off that produces the highest degree of attenuation in the measured peak MFL amplitude. The curves indicate that the majority of the measured reduction in MFL amplitude is experienced in the first increment (0.125 inch) of lift-off. It strongly indicates that low levels of sensor lift-off significantly reduce the measured peak MFL amplitude which is contrary to the industry assumption that "a little bit of debris won't affect the MFL data." Once any lift-off (especially of the sensors or sensors and magnetizer simultaneously) has occurred, the majority of the signal degradation is already done and the amount of additional signal reduction decreases with increasing lift-off. This observation was consistent with all defect types examined.

\subsection{DISCUSSION TOPICS}

The following paragraphs address issues that deserve further mention.

\subsubsection{END EFFECTS}

End effects a term that is used to refer to the difference in baseline MFL amplitude on the fore and aft sides of the defect (Figure 47). Review of any of the graphs in the Appendix 11-19 show that the baseline amplitudes for the same axial distance from the axial centerline of the defect do not match. This occurred because the geometry of the coupon is not consistent for the length of pipe coupon inspected. The baseline amplitude of the curve is higher on the side of the defect that is closest to the end of the pipe coupon. This is because there is less material through which the magnetic flux can permeate. Therefore, the baseline MFL readings are higher. Figure 47 illustrates this effect. This has no effect on the measured peak MFL amplitude. However, it could potentially cause an axial shift in the location of the peak amplitude.

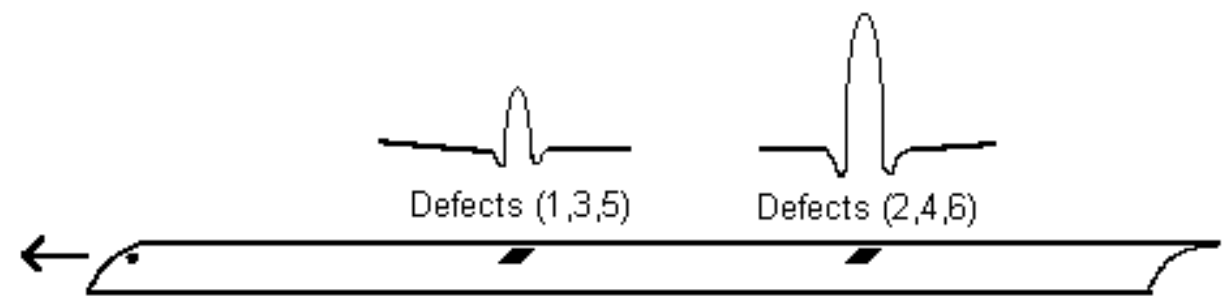

Figure 47 - End effects 


\subsubsection{CHANGE IN BASELINE FLUX LEAKAGE MEASUREMENTS AS COMPARED TO GRI RESULTS}

Review of the test data showed that the baseline MFL amplitudes decreased with increasing lift-off of both the sensors and magnetizer simultaneously. This is in contrast to GRI's results [16]. The testing conducted as part of this research was conducted on a pipe coupon with only a single section of a magnetizing assembly. Their tests were conducted with an actual multi-channel MFL inspection tool on the entire circumference of a section of pipe. With the adjacent magnetizing sections available, higher levels of magnetization can be attained. This is most likely the factor which led to the observation of an increase in baseline MFL amplitudes.

\subsubsection{ANALOG OUTPUT OF THE GAUSSMETER}

The gaussmeter used for this testing procedure had 3 possible ranges that could be used (100 Gauss, 1000 Gauss, and 10,000 Gauss). The gaussmeter is capable of $100 \%$ over range. Therefore the gaussmeter can measure up to 200 Gauss in the 100 Gauss range and 2000 Gauss in the 1000 Gauss range. In addition, the gaussmeter specifications state that the analog output is capable of a $\pm 1.000 \mathrm{~V}$ or $\pm 2.000 \mathrm{~V}$ at $100 \%$ over range.

The 100 Gauss range was selected for testing because the baseline flux leakage readings ranged from 88 to 105 Gauss. The measured peak MFL amplitude varied with defect type. The widest defect (defect 6) produced the greatest flux leakage amplitude (373 Gauss). This was clearly in excess of 200 Gauss.

The gaussmeter manufacturer, Walker Scientific, Inc., was consulted and they indicated that the gaussmeter will produce an analog output voltage in excess of $\pm 2.000 \mathrm{~V}$, but they only publish accuracy specifications up to $\pm 2.000 \mathrm{~V}$. However, they stated that the accuracy of the gaussmeter will remain within published specifications up to approximately 400 Gauss or $\pm 4.000 \mathrm{~V}$. Therefore, the acquired flux leakage data is still within the tolerances set forth in the specifications. 


\section{CHAPTER 8. CONCLUSIONS \& RECOMMENDATIONS}

\subsection{CONCLUSIONS}

The test results have shown very convincingly that debris-induced lift-off, in all its forms (either sensor, magnetizer, or both simultaneously), can have a significant effect on MFL data. Since there is no direct method currently available to detect lift-off, it will always be a potential problem for MFL inspections. Simultaneous sensor and magnetizer lift-off causes the greatest reduction in peak MFL amplitudes. Therefore, layers of tightly adhered debris should be a primary concern for pipeline operators.

Lift-off of the sensors (alone) has been shown to produce nearly the same magnitude of signal attenuation as simultaneous sensor and magnetizer lift-off for most cases. In addition, it occurs the easiest, the most often, and is the very difficult to detect. Hence, the removal of loose debris from the pipeline system should also be a priority for pipeline operators.

The peak and baseline MFL amplitudes have been shown to decrease as a result of all forms of debris-induced lift-off. Deeper and wider defects experience the largest percentage of peak MFL amplitude reduction with lift-off. It is these defects that pipeline operators are attempting to locate when conducting MFL inspections. These reductions in the MFL signal have the potential to cause small and moderately sized defects to be missed and larger defects to be severely underestimated in size. Both cases are undesirable and can have a significant impact on the integrity of the pipeline.

Perhaps the most noteworthy conclusion is the observation that very low levels of liftoff can cause significant reductions in the measured MFL amplitude. It has long been thought that "a little bit of debris won't affect the MFL data." However, the results of this research has shown this assumption to be false. Adherence to this doctrine invites the potential for a compromise in the integrity of the pipeline.

This research has also concluded that pipeline cleanliness is a significant factor in the quality of MFL inspection data. Therefore, the onus is on pipeline operators to take deliberate steps to insure pipeline cleanliness in advance of MFL inspections. This improves both the probability of detection and accuracy of the signal characterization.

\subsection{RECOMMENDATIONS}

Listed below are recommendations that could help reduce the effects of debrisinduced lift-off. Also included are recommendations which could further the understanding of debris-induced lift-off.

1. Pipeline operators should perform regular pipeline cleaning to prevent large accumulations of debris (especially layers of tightly adhered debris).

2. Pipeline operators should use advanced pipeline cleaning methods (online chemical cleaning or gel cleaning) when conventional methods fail to provide inspection cleanliness.

3. Inspection vendors should develop and implement technologies that specifically detect and measure the level of lift-off experienced by the corrosion sensors and magnetizer. The design of the sensor arm could be improved to simulate the action of a four-bar linkage. This would preclude the additional signal degradation due to the angular 
displacement and additional lift-off created due the sensor head not being parallel to the pipe surface.

4. Perform further research and testing to better further understand the effect of debrisinduced lift-off on MFL inspection results. 


\section{REFERENCES}

1. American Society of Mechanical Engineers, “ASME B31G-1991 (Revision of ANSI/ASME B31G-1984) Manual for Determining the Remaining Strength of Corroded Pipelines," 1991.

2. American Society for Nondestructive Testing, Nondestructive Testing Handbook, Second Edition, The American Society of Nondestructive Testing, 1986.

3. Avallone, Eugene A. and Baumeister III, Theodore, Marks' Standard Handbook for Mechanical Engineers, $10^{\text {th }}$ Edition, McGraw-Hill, 1996.

4. Crouch, A.E., "In-Line Inspection of Natural Gas Pipelines,” GRI Report 91-0365.

5. Doebelin, Ernest O., Measurement Systems - Fourth Edition, McGraw-Hill, 1990.

6. Kershaw, Christopher F., "The Importance of Enhanced Cleaning for In-line Inspection," The Pipeline Pigging Conference, Houston, TX, Feb 3-6, 1997.

7. Kiefner, J.F. and Vieth, P.H., "A Modified Criterion for Evaluating the Remaining Strength of Corroded Pipe," Final Report on Project PR 3-805 to the Pipeline Corrosion Supervisory Committee of the Pipeline Research Committee of the American Gas Association, December 1989.

8. Nestleroth, J.B., et al., "Magnetic Flux Leakage (magnetic flux leakage) Technology for Natural Gas Pipeline Inspection", update to Gas Research Institute Report GRI-91/0367, to be published.

9. Payne, Larry, "Cleaning and Geometry Inspection Prior to Corrosion Inspection," The Pipeline Pigging Conference, Kuala Lumpur, Malaysia, June 291998 - July 2, 1998.

10. Posakony, G.J., "Advances in Nondestructive Evaluation Technology and Their Possible Application in Gas Transmission Pipeline Inspection,” GRI Report 91/0368, 1992.

11. Posakony, G.J. and Hill, V.L., "Assuring the Integrity of Natural Gas Transmission Pipelines,” GRI Report 91/0366.

12. Taylor, John R., An Introduction to Error Analysis - Second Edition, University Science Books, 1997.

13. Tiratsoo, John H., Pipeline Pigging Technology, 2nd Edition, Gulf Publishing Companyand Scientific Surveys Ltd., Houston, TX, 1991.

14. Turner, John and Hill, Martyn., Instrumentation for Engineers and Scientists, Oxford University Press, New York, 1999. 
15. Valentine, Francisco, "Introduction to Online Chemical Cleaning of Natural Gas Pipelines", Pipeline Pigging and Integrity Assessment Conference, Stavanger, Norway, June 15-17, 1999.

16. Valentine, Francisco, "Effect of Pipeline Debris on Geometry Tool Inspection Results", Pipeline \& Gas Journal, January 2000.

17. Valentine, Francisco and Luedke, Jonathan, "Effect of Pipeline Debris on Magnetic Flux Leakage Inspection Results", Pipeline Pigging and Integrity Assessment Conference, Houston, TX, February 1-2, 2000.

18. http://www.pipetronix.com

19. http://www.dataq.com

20. http://www. spaceagecontrols.com

21. http://www.walkerscientific.com 


\section{APPENDICES}

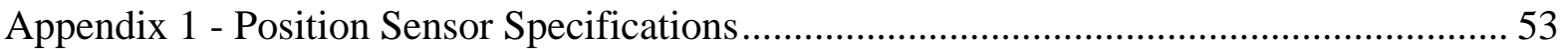

Appendix 2 - Gaussmeter Specifications \& Drawings ............................................. 58

Appendix 3 - Data Acquisition System Specifications ................................................. 64

Appendix 4 - Magnet Specifications \& Drawings ..................................................6. 65

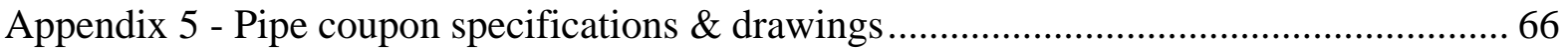

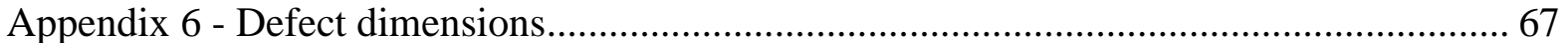

Appendix 7 - Sensor holder specifications \& drawings............................................. 68

Appendix 8 - Coupon guides specifications \& drawings............................................ 69

Appendix 9 - Displacement device specifications \& drawings .................................... 70

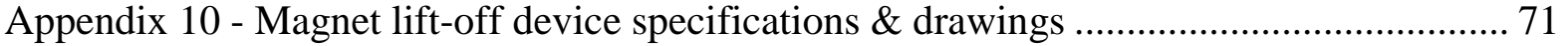

Appendix 11 - Sensor lift-off graphs (zero magnetizer lift-off) ................................... 72

Appendix 12 - Magnetizer lift-off graphs (zero sensor lift-off) ................................. 75

Appendix 13 - Simultaneous sensor and magnetizer lift-off graphs............................. 78

Appendix 14 - Defect 1 - Magnetic flux leakage signal comparison graphs ..................... 81

Appendix 15 - Defect 2 - Magnetic flux leakage signal comparison graphs ..................... 84

Appendix 16 - Defect 3 - Magnetic flux leakage signal comparison graphs ..................... 87

Appendix 17 - Defect 4 - Magnetic flux leakage signal comparison graphs ..................... 90

Appendix 18 - Defect 5 - Magnetic flux leakage signal comparison graphs ......................93

Appendix 19 - Defect 6 - Magnetic flux leakage signal comparison graphs ....................... 96

Appendix 20 - Peak magnetic flux leakage amplitude vs. lift-off of sensor, magnetizer, and

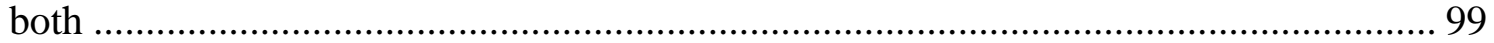

Appendix 21 - Percent reduction in magnetic flux leakage amplitude vs. increment of lift-off

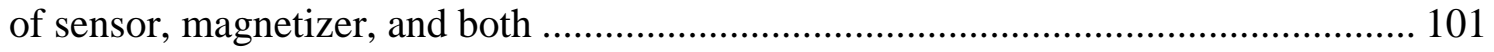

Appendix 22 - Baseline magnetic flux leakage amplitude vs. lift-off of sensor, magnetizer,

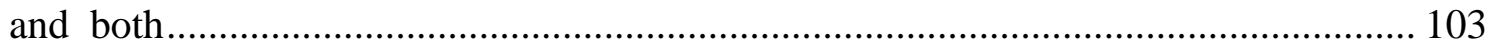

Appendix 23 - Percent reduction in baseline magnetic flux leakage amplitude vs. increment

of lift-off of sensor, magnetizer, and both ...................................................... 105 


\section{DATA SHEET \\ Series 160, $161 \& 162$ \\ Spraces Age Contrah, Inc.}

Analog-Output Miniature Position Transducers

$\mathrm{SO21H}(\mathrm{H})$ : page 1 of 5

KEY FEATURES

- 42.5-inch (1080-mm) Maximum Travel (Series 162)

- Analog Signal Using Precision Conductive Plastic/Hybrid Potentiometers

- Threaded Drum for Enhanced Repeatability

- Bearing-Mounted Rotating Components

- Optional Flexible Mounting Bases

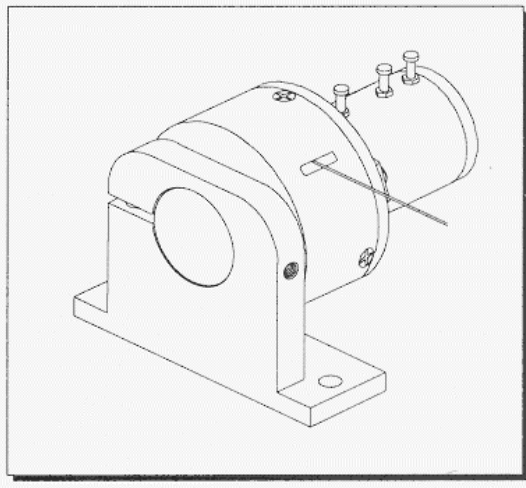

\section{POTENTIOMETER SPECIFICATIONS}

Potentiometer Type

Resistance: Value, Tolerance

Travel: Electrical, Mechanical

Mechanical Life

Power Rating

Max. Indep. Linearity Error

Output Smoothness

Insulation Resistance

Dielectric Strength

Resolution

Operating Temperature

Electrical Connection

Shock

Vibration

Temperature Coefficient

\section{OTHER SPECIFICATIONS}

Case Materials

Displacement Cable

Electrical Connections

Approximate Weight

Environmental Sealing

\section{1-turn}

1-turn, conductive plastic

$5 \mathrm{~K}$ ohms, $\pm 20 \%$ $340^{\circ}, 360^{\circ}$

10 million shaft revolutions

1.0 watts at $158^{\circ} \mathrm{F}\left(70^{\circ} \mathrm{C}\right)$

$\pm 1.0 \%$ per VRCI-P- $100 \mathrm{~A}$

$$
<0.1 \%
$$

100 Mohms

1000 volts RMS

infinite signal

$-40^{\circ}$ to $257^{\circ} \mathrm{F}\left(-40^{\circ}\right.$ to $\left.125^{\circ} \mathrm{C}\right)$

3-terminal $(1,2,3)$

$100 \mathrm{~g}$ for $6 \mathrm{~ms}$

10 to $2000 \mathrm{~Hz}$ at $15 \mathrm{~g}$

$\pm 222 \mathrm{ppm} /{ }^{\circ} \mathrm{F}\left( \pm 400 \mathrm{ppm} /{ }^{\circ} \mathrm{C}\right)$ 3-turn

3-turn, hybrid construction

$5 \mathrm{~K}$ ohms, $\pm 5 \%$

$1080^{\circ}, 1080^{\circ}+10^{\circ}-0^{\circ}$

5 million shaft revolutions

1.5 watts at $158^{\circ} \mathrm{F}\left(70^{\circ} \mathrm{C}\right)$

$\pm 0.5 \%$ per VRCI-P- $100 \mathrm{~A}$

$0.5 \% \max$.

1000 Mohms

1000 volts RMS

infinite signal

$-67^{\circ}$ to $257^{\circ} \mathrm{F}\left(-55^{\circ}\right.$ to $125^{\circ} \mathrm{C}$ )

3-terminal (CW, CCW, S)

$100 \mathrm{~g}$ for $6 \mathrm{~ms}$

10 to $2000 \mathrm{~Hz}$ at $15 \mathrm{~g}$ $\pm 389 \mathrm{ppm} /{ }^{\circ} \mathrm{F}\left( \pm 700 \mathrm{ppm} /{ }^{\circ} \mathrm{C}\right)$ 5-turn

5-turn, hybrid construction $5 \mathrm{~K}$ ohms, $\pm 5 \%$ $1800^{\circ}, 1800^{\circ}+10^{\circ}-0^{\circ}$ 5 million shaft revolutions 2.0 watts at $158^{\circ} \mathrm{F}\left(70^{\circ} \mathrm{C}\right)$ $\pm 0.35 \%$ per VRCI-P- $100 \mathrm{~A}$

$0.35 \%$ max.

1000 Mohms 1000 volts RMS infinite signal $-67^{\circ}$ to $257^{\circ} \mathrm{F}\left(-55^{\circ}\right.$ to $\left.125^{\circ} \mathrm{C}\right)$ 3-terminal (CW, CCW, S) $100 \mathrm{~g}$ for $6 \mathrm{~ms}$

10 to $2000 \mathrm{~Hz}$ at $15 \mathrm{~g}$ $\pm 389 \mathrm{ppm} /{ }^{\circ} \mathrm{F}\left( \pm 700 \mathrm{ppm} /{ }^{\circ} \mathrm{C}\right)$

precision-machined anodized 2024 aluminum

0.018-inch (0.46-mm) dia., 7-by-7 stranded stainless steel, 40-lb (177-N) min. breaking strength. A minimum of 12 inches $(305 \mathrm{~mm}$ ) of displacement cable is provided with an uncrimped eyelet and swivel for connection to the application. Swivel minimum yield strength is $9 \mathrm{lbs}(40 \mathrm{~N})$. Other connecting solutions available on request.

Three solder terminals. Customer-specified electrical cable and connectors available upon request.

\begin{tabular}{|l|ll|ll|}
\hline \multicolumn{2}{|c|}{ Series 160} & \multicolumn{2}{|c|}{ Series 161} & \multicolumn{2}{c|}{ Series 162} \\
\hline 4 oz. $113 \mathrm{~g}$ & $6.1 \mathrm{oz}$. & $170 \mathrm{~g}$ & $9 \mathrm{oz}$. & $255 \mathrm{~g}$ \\
\hline
\end{tabular}

NEMA 4/IP 56 (with optional sensor cover)

A Small, Flexible Alternative to LVDTS and Linear Potentiometers 


\section{DATA SHEET \\ Series $160,161 \& 162$ \\ SpreceAge Cantral, Inc. \\ Analog-Output Miniature Position Transducers \\ $\mathrm{S} 021 \mathrm{H}(\mathrm{H})$ : page 2 of 5}

MODEL NUMBERS AND CABLE TENSIONS: Series 160

\begin{tabular}{|c|c|c|c|c|c|c|c|c|c|c|}
\hline \multirow[t]{3}{*}{ Model } & & \multicolumn{8}{|c|}{ Cable Tension Range (Full Retraction to FullExtraction) } \\
\hline & & & \multicolumn{2}{|c|}{ Opt 111: -050 spring } & \multicolumn{2}{|c|}{ Opt 112: -060 spring } & \multicolumn{2}{|c|}{ Opt 113: -070 spring } & \multicolumn{2}{|c|}{ Opt 114: -080 spring } \\
\hline & inches & $\mathrm{mm}$ & oz. & $\mathrm{N}$ & oz. & $\mathrm{N}$ & oz. & $\mathrm{N}$ & oz. & $\mathrm{N}$ \\
\hline $160-0121$ & 1.50 & 38 & 14 to 18 & 4 to 5 & - & - & - & - & - & - \\
\hline $160-0151$ & 1.88 & 48 & 8 to 15 & 2 to 4 & - & - & - & - & - & - \\
\hline $160-0161$ & 2.00 & 51 & 9 to 15 & 3 to 4 & 16 to 25 & 4 to 7 & 30 to 37 & 8 to 10 & 40 to 55 & 11 to 15 \\
\hline $160-0181$ & 2.25 & 57 & 8 to 13 & 2 to 4 & 16 to 25 & 4 to 7 & 30 to 40 & 8 to 11 & 40 to 55 & 11 to 15 \\
\hline $160-0201$ & 2.50 & 64 & 9 to 11 & 3 to 3 & 16 to 25 & 4 to 7 & 30 to 40 & 8 to 11 & 40 to 55 & 11 to 15 \\
\hline $160-0231$ & 2.88 & 73 & 7 to 11 & 2 to 3 & 16 to 25 & 4 to 7 & 30 to 40 & 8 to 11 & 40 to 55 & 11 to 15 \\
\hline $160-0241$ & 3.00 & 76 & $z$ to 10 & 2 to 3 & 11 to 16 & 3 to 4 & 60 to 80 & 17 to 22 & 40 to 55 & 11 to 15 \\
\hline $160-0261$ & 3.25 & 83 & $z$ to 9 & 2 to 3 & 10 to 12 & 3 to 3 & 25 to 35 & 7 to 10 & 40 to 55 & 11 to 15 \\
\hline $160-0281$ & 3.50 & 89 & 6 to 10 & 2 to 3 & 16 to 25 & 4 to 7 & 25 to 35 & 7 to 10 & 40 to 55 & 11 to 15 \\
\hline $160-0311$ & 3.88 & 98 & 6 to 8 & 2 to 2 & 16 to 25 & 4 to 7 & 25 to 35 & 7 to 10 & 40 to 55 & 11 to 15 \\
\hline $160-0321$ & 4.00 & 102 & 4 to 8 & 1 to 2 & 5 to 18 & 1 to 5 & 12 to 20 & 3 to 6 & 36 to 64 & 10 to 18 \\
\hline $160-0403$ & 5.00 & 127 & 12 to 28 & 3 to 8 & 24 to 34 & 7 to 10 & 45 to 60 & 13 to 17 & 112 to 192 & 31 to 53 \\
\hline $160-0483$ & 6.00 & 152 & 10 to 21 & 3 to 6 & 14 te 30 & 4 to 8 & 45 to 70 & 13 to 20 & 93 to 160 & 26 to 45 \\
\hline $160-0523$ & 6.50 & 165 & 10 to 20 & 3 to 6 & 16 to 32 & 4 to 9 & 45 to 60 & 13 to 17 & 86 to 147 & 24 to 41 \\
\hline $160-0563$ & 7.00 & 178 & 10 to 18 & 3 to 5 & 15 to 30 & 4 to 8 & 20 to 45 & 6 to 13 & 80 to 137 & 22 to 38 \\
\hline $160-0643$ & 8.00 & 203 & $z$ to 17 & 2 to 5 & 9 to 16 & 3 to 4 & 24 to 37 & 7 to 10 & 70 to 120 & 19 to 33 \\
\hline $160-0675$ & 8.38 & 213 & 15 to 30 & 4 to 8 & 16 to 35 & 4 to 10 & 20 to 45 & 6 to 13 & 66 to 115 & 18 to 32 \\
\hline $160-0723$ & 9.00 & 229 & - & - & 12 to 20 & 3 to 6 & 20 to 40 & 6 to 11 & 62 to 110 & 17 to 31 \\
\hline $160-0773$ & 9.63 & 244 & - & - & 16 to 29 & 4 to 8 & 20 to 40 & 6 to 11 & 59 to 105 & 16 to 29 \\
\hline $160-0803$ & 10.00 & 254 & 6 to 12 & 2 to 3 & 10 to 18 & 3 to 5 & 19 te 32 & 5 to 9 & 38 to 124 & 11 to 34 \\
\hline $160-0815$ & 10.13 & 257 & - & - & 19 to 34 & 5 to 9 & 30 to 72 & 8 to 20 & 50 to 142 & 14 to 40 \\
\hline $160-0875$ & 10.88 & 276 & 10 to 20 & 3 to 6 & 16 to 28 & 4 to 8 & 24 to 55 & 7 to 15 & 51 to 92 & 14 to 26 \\
\hline $160-0893$ & 11.13 & 283 & - & - & 16 to 27 & 4 to 8 & 20 to 40 & 6 to 11 & 48 to 88 & 13 to 24 \\
\hline $160-0945$ & 11.75 & 298 & - & - & 13 to 26 & 4 to 7 & 20 to 40 & 6 to 11 & 45 to 84 & 13 to 23 \\
\hline $160-0963$ & 12.00 & 305 & 5 to 11 & 1 to 3 & $z$ to 19 & 2 to 5 & 17 to 27 & 5 to 8 & 30 to 80 & 8 to 22 \\
\hline $160-0993$ & 12.38 & 314 & 6 to 10 & 2 to 3 & 12 to 30 & 3 to 8 & 16 to 20 & 4 to 6 & 38 to 77 & 11 to 21 \\
\hline $160-1085$ & 13.50 & 343 & 10 to 20 & 3 to 6 & 12 to 22 & 3 to 6 & 20 to 53 & 6 to 15 & 35 to 133 & 10 to 37 \\
\hline $160-1215$ & 15.13 & 384 & 5 to 16 & 1 to 4 & 10 to 19 & 3 to 5 & 21 to 45 & 6 to 13 & 27 to 108 & 8 to 30 \\
\hline $160-1285$ & 16.00 & 406 & 8 to 15 & 2 to 4 & 9 to 23 & 3 to 6 & 19 to 42 & 5 to 12 & 20 to 102 & 6 to 28 \\
\hline $160-1345$ & 16.75 & 425 & $z$ to 15 & 2 to 4 & 9 to 17 & 3 to 4 & 17 to 40 & 5 to 11 & 24 to 96 & 7 to 27 \\
\hline $160-1505$ & 18.75 & 476 & 3 to 11 & 1 to 3 & 9 to 17 & 3 to 5 & 18 to 39 & 5 to 11 & 21 to 86 & 6 to 24 \\
\hline $160-1615$ & 20.13 & 511 & 6 to 14 & 2 to 4 & 9 to 17 & 3 to 5 & 16 to 35 & 4 to 10 & 18 to 90 & 5 to 25 \\
\hline $160-1705$ & 21.25 & 540 & 6 to 11 & 2 to 3 & 8 to 17 & 2 to 5 & 15 to 33 & 4 to 9 & 14 to 75 & 4 to 21 \\
\hline
\end{tabular}

Bolded entries are standard cable tension and will be specified unless overridden on purchase order.

A Small, Flexible Alternative to LVDTs and Linear Potentiometers 


\section{DATA SHEET \\ Series $160,161 \& 162$ \\ SpreceAge Cantrol, Inc. \\ Analog-Output Subminiature Position Transducers \\ S021H(H): page 3 of 5}

MODEL NUMBERS AND CABLE TENSIONS: Series 161 and 162

\begin{tabular}{|c|c|c|c|c|c|c|c|c|c|c|}
\hline \multirow[t]{3}{*}{ Model } & \multirow{2}{*}{\multicolumn{2}{|c|}{ Range }} & \multicolumn{8}{|c|}{ Cable Tension Range (Full Retraction to FullExtraction) } \\
\hline & & & \multicolumn{2}{|c|}{ Opt 111: -050 spring } & \multicolumn{2}{|c|}{ Opt 112: -060 spring } & \multicolumn{2}{|c|}{ Opt 113: -070 spring } & \multicolumn{2}{|c|}{ Opt 114: -080 spring } \\
\hline & inches & $\mathrm{mm}$ & oz. & $\mathrm{N}$ & oz. & $\mathrm{N}$ & oz. & $\mathrm{N}$ & oz. & $\mathrm{N}$ \\
\hline $161-0361$ & 4.50 & 114 & - & - & 18 to 30 & 5 to 8 & 20 to 30 & 6 to 8 & 40 to 65 & 11 to 18 \\
\hline $161-0411$ & 5.13 & 130 & - & - & 18 to 30 & 5 to 8 & 20 to 30 & 6 to 8 & 35 to 50 & 10 to 14 \\
\hline $161-0441$ & 5.50 & 140 & - & - & 18 to 30 & 5 to 8 & 20 to 30 & 6 to 8 & 35 to 50 & 10 to 14 \\
\hline $161-0461$ & 5.75 & 146 & - & - & 18 to 30 & 5 to 8 & 20 to 30 & 6 to 8 & 35 to 50 & 10 to 14 \\
\hline $161-1143$ & 14.25 & 362 & - & - & 6 to 12 & 2 to 3 & 20 to 35 & 6 to 10 & 30 to 60 & 8 to 17 \\
\hline $161-1283$ & 16.00 & 406 & - & - & 6 to 11 & 2 to 3 & 12 te 25 & 3 to 7 & 20 to 65 & 6 to 18 \\
\hline $161-1393$ & 17.38 & 441 & - & - & - & - & 10 to 16 & 3 to 4 & 20 to 40 & 6 to 11 \\
\hline $161-1443$ & 18.00 & 457 & - & - & - & - & 10 to 18 & 3 to 5 & 20 to 55 & 6 to 15 \\
\hline $161-1915$ & 23.88 & 606 & - & - & $z$ to 14 & 2 to 4 & 13 to 30 & 4 to 8 & 20 to 80 & 6 to 22 \\
\hline $161-2145$ & 26.75 & 679 & - & - & - & - & 16 to 95 & 4 to 26 & 16 to 95 & 4 to 26 \\
\hline $161-2325$ & 29.00 & 737 & - & - & - & - & 10 to 16 & 3 to 4 & 15 to 60 & 4 to 17 \\
\hline $161-2405$ & 30.00 & 762 & - & - & - & - & 9 to 20 & 3 to 6 & 12 to 60 & 3 to 17 \\
\hline $162-0521$ & 6.50 & 165 & - & - & 10 to 16 & 3 to 4 & 15 te 25 & 4 to 7 & 20 to 32 & 6 to 9 \\
\hline $162-0561$ & 7.00 & 178 & - & - & 10 to 16 & 3 to 4 & 20 to 30 & 6 to 8 & 35 to 55 & 10 to 15 \\
\hline $162-0621$ & 7.75 & 197 & - & - & 10 to 16 & 3 to 4 & 20 to 30 & 6 to 10 & 35 to 55 & 10 to 15 \\
\hline $162-0651$ & 8.13 & 206 & - & - & 10 to 16 & 3 to 4 & 20 to 30 & 6 to 10 & 35 to 55 & 10 to 15 \\
\hline $162-1643$ & 20.50 & 521 & - & - & 10 to 16 & 3 to 4 & 13 to 34 & 4 to 9 & - & - \\
\hline $162-1763$ & 22.00 & 559 & - & - & 10 to 16 & 3 to 4 & 13 to 32 & 4 to 9 & - & - \\
\hline $162-1923$ & 24.00 & 610 & - & - & - & - & 12 to 30 & 3 to 8 & 25 to 40 & 7 to 11 \\
\hline $162-2043$ & 25.50 & 648 & - & - & - & - & 2 to 13 & 3 to 4 & 15 to 30 & 4 to 8 \\
\hline $162-2735$ & 34.13 & 867 & - & - & - & - & 9 to 21 & 3 to 6 & 25 to 40 & 7 to 11 \\
\hline $162-2945$ & 36.75 & 933 & - & - & - & - & 8 to 20 & 2 to 6 & 20 to 35 & 6 to 10 \\
\hline $162-3205$ & 40.00 & 1016 & - & - & - & - & $Z$ te 18 & 3 to 5 & 12 to 43 & 3 to 12 \\
\hline $162-3405$ & 42.50 & 1080 & - & - & 4 to 14 & 1 to 4 & $z$ to 17 & 2 to 5 & 10 to 40 & 3 to 11 \\
\hline
\end{tabular}

Bolded entries are standard cable tension and will be specified unless overridden on purchase order.

A Small, Flexible Alternative to LVDTs and Linear Potentiometers 


\section{DATA SHEET \\ Series $160,161 \& 162$ \\ SpreceAge Contral, Inc. \\ Analog-Output Subminiature Position Transducers

OPTIONS: Series $160,161 \& 162$

$\begin{array}{ll}\text { Opt. } 111 & \text { cable tension: }-050 \\ \text { Opt. } 112 & \text { cable tension: }-060 \\ \text { Opt. } 113 & \text { cable tension: }-070 \\ \text { Opt. } 114 & \text { cable tension: }-080 \\ \text { Opt. } 9 & \text { SPECIAL }=\text { (describe special requirement or specification to be met) }\end{array}$

OPTIONS: Series 160 (sensor cover, cable exit, bases)
Opt. C16
Opt. $\mathrm{C} 17$
cable exit: slot (_60)
Opt. C18
cable exit: cable guide (_60); pn 160045-1
Opt. C24
cable exit: idler (_60); pn 160022; cannot be installed with cable guide
Opt. $\mathrm{C} 27$
base: mounting disk (_6_); 160040-1
Opt. C30
base: standard (_60); pn 160015-1
Opt. C33
base: universal (_60); pn 160030-1
Opt. C35
base: big foot (_60/_61); pn 160015-13
base: h (_60); pn 160015-G1
Opt. $\mathrm{C} 10$
no sensor cover ( 60$)$
Opt. C11
sensor cover (_60); pn 160060

OPTIONS: Series 161 (sensor cover, cable exit, bases)
Opt. C19
cable exit: slot (_61)
Opt. C20
cable exit: cable guide (_61); pn 160045-3
Opt. C21
Opt. C24
base: mounting disk (_6_); 160040-1
Opt. C28
base: standard (_61); pn 160015-3
Opt. C31
base: universal (_61); pn 160030-3
Opt. C33
Opt. $\mathrm{C} 12$
base: big foot (_60/_61); pn 160015-13
no sensor cover $\left(\_61\right)$
Opt. C13
sensor cover (_61); pn 160060

cable exit: idler (611_62); pn 161022; cannot be installed with cable guide

OPTIONS: Series 162 (sensor cover, cable exit, bases)
Opt. C22
cable exit: slot (_62)
Opt. C23
cable exit: cable guide (_62); pn 160045-5
Opt. C37
cable exit: idler (_61/_62); pn 161022; cannot be installed with cable guide
Opt. C24
base: mounting disk (_6_); 160040-1
Opt. C29
base: standard (_65); pn 160015-5
Opt. C32
base: universal (_62); pn 160030-5
Opt. C34
base: big foot (_62); pn 160015-15
Opt. C14
no sensor cover (_62)
Opt. C15
sensor cover (_62); pn 160060

A Small, Flexible Alternative to LVDTs and Linear Potentiometers 


\section{DATA SHEET}

Series $160,161 \& 162$

Spaceflge Control, Inc.

Analog-Output Subminiature Position Transducers

$\mathrm{S} 021 \mathrm{H}(\mathrm{H})$ : page 5 of 5

DIMENSIONS: shown with slot cable exit, standard base, and optional sensor cover.
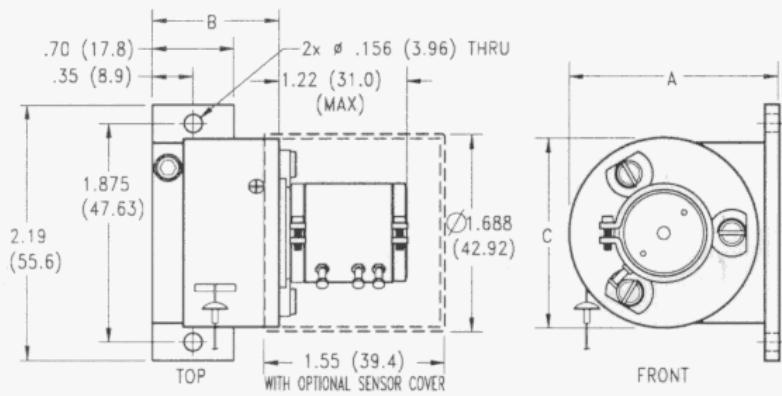

\begin{tabular}{|c|c|c|c|}
\hline SERIES & A & B & c \\
\hline 160 & $1.81(46.0)$ & $1.07(27.2)$ & $1.62(41.1)$ \\
\hline 161 & $2.43(61.7)$ & $1.07(27.2)$ & $2.24(56.9)$ \\
\hline 162 & $3.19(81.0)$ & $1.07(27.2)$ & $2.99(75.9)$ \\
\hline \multicolumn{4}{|c|}{ ALL DINENSIONS ARE IN ACHES (UW] } \\
\hline \multicolumn{2}{|c|}{ smviL - } & ELECTRICAL & TRECAL SCHEMATC \\
\hline \multicolumn{2}{|c|}{$\begin{array}{c}\text { CnOD00 } \\
-1.00(25.4) \mid\end{array}$} & $5^{0 \text { aro }}$ & ORe (1) of cw) \\
\hline
\end{tabular}

DIMENSIONS: shown with cable guide cable exit, standard base, and optional sensor cover.
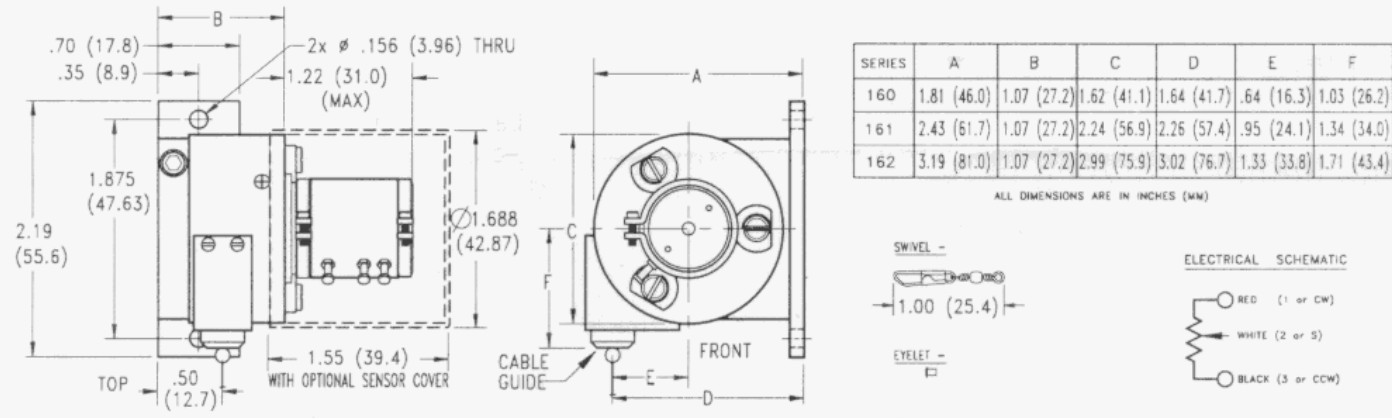

DIMENSIONS: shown with idler cable exit, standard base, and optional sensor cover.

Note: Idler cable exit will reduce displacement cable life and may reduce maximum range by up to 0.75 inch (19.1 mm).
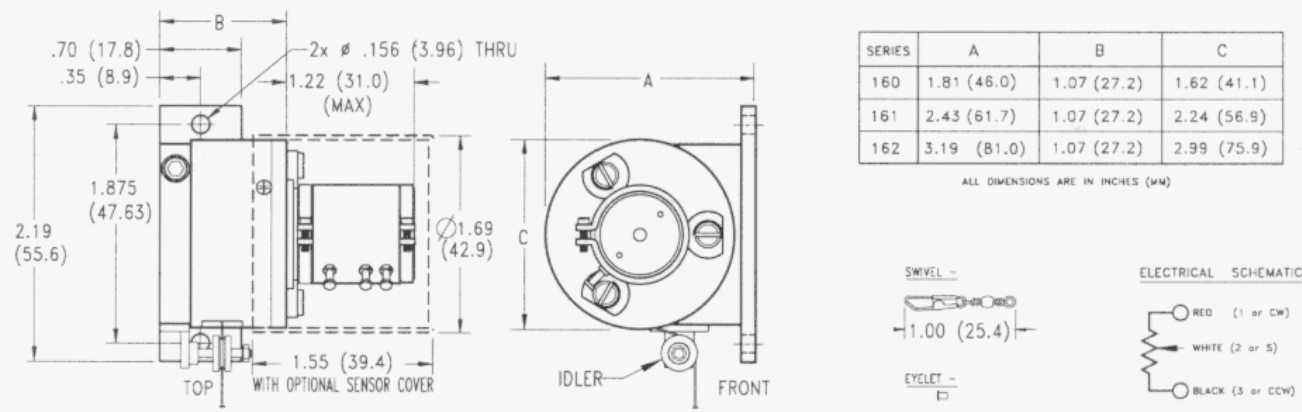

A Small, Flexible Alternative to LVDTs and Linear Potentiometers 
Appendix 2 - Gaussmeter Specifications \& Drawings

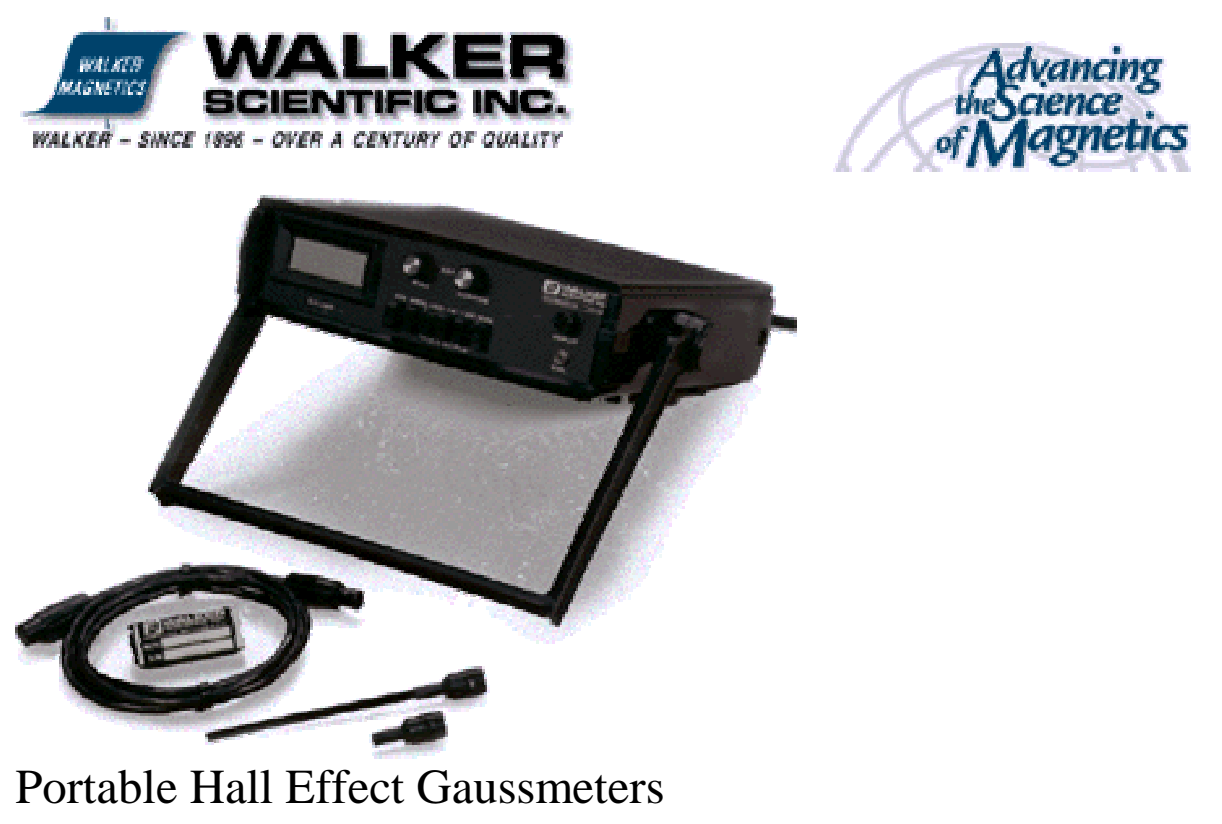

MG-5D/MG-5DAR/MG-5DP

General:

The MG-5 Series are general purpose portable Hall effect gaussmeters designed to measure both DC \& AC (RMS) magnetic fields.

Three full-scale bipolar ranges of \pm 100.0 gauss, $\pm 1.000 \mathrm{kG}$ and $\pm 10.00 \mathrm{kG}$ with $100 \%$ over-range and resolution of $0.05 \%$ provides DC \& AC field readings from \pm 100 milligauss to $\pm 19.99 \mathrm{kG}$ with true RMS readings from $3 \mathrm{~Hz}$ to $20 \mathrm{kHz}$; readings are displayed on a $31 / 2$ digit $\pm 0.1 \%$ bipolar LCD meter.

Because the MG-5D is a Hall effect instrument, a Hall probe is required to couple with and measure the magnetic field. A wide selection of precalibrated transverse and axial Hall probes is available to meet most every application, including probes which will extend the measuring range of this instrument to $150.0 \mathrm{kG}$.

The MG-5 Series operates either from AC or from sealed lead acid batteries. During AC operation, the batteries receive a trickle charge which keeps them fresh until the instrument is required for portable use. Freshly charged batteries will continuously operate this instrument for approximately 10 hours.

In addition, an analog output is also provided for external monitoring.

MG-5DAR In the auto-range mode, the MG-5DAR will automatically switch to the appropriate full-scale range to accurately display the measured field and provide optimum field measurement resolution.

A reading of more than \pm 1999 counts will automatically switch this unit to a higher range and a reading of less than \pm 100 counts will automatically switch this unit to a lower range. The decimal point indicates the operating scale.

MG-5DP In the Peak Mode, the MG-5DP will sense and display the most recent peak magnetic field level from $\mathrm{DC}$ to $20 \mathrm{kHz}$. 
This instrument can be set to either detect the peak value when the field is bipolar (varying from positive to negative) or it can be set to exclusively detect either the positive peak or the negative peak of a varying field. Because of the digital circuit design, there is no decay in the peak field reading.

Applications:

Measure Residual Fields

Analyze Magnetic Circuits and Components

Classify Magnets

Measure Absolute and Differential Fields

Plot Field Uniformity

Measure Stray \& Leakage Fields

Features:

3 1/2 Digit $0.1 \%$ Bipolar Display

DC \& AC Fields, \pm 100 milligauss to $\pm 19.99 \mathrm{kG}$ with $1 \mathrm{X}$ probes

Range Extendable to $150.0 \mathrm{kG}$ with Select Probes

True RMS Readings to $20 \mathrm{kHz}$

Wide Selection of Precalibrated Probes: 1X, 10X and 100X

Operates with either AC or Battery; Fully Portable

Analog Output; For External Monitoring

Automatic range switching for optimum field measurement resolution; MG-5DAR only

Positive and/or negative peak reading; MG-5DP only

No field decay in Peak Mode; MG-5DP only

High Impact Plastic Case with Carrying Handle

One Year Warranty

Accessories:

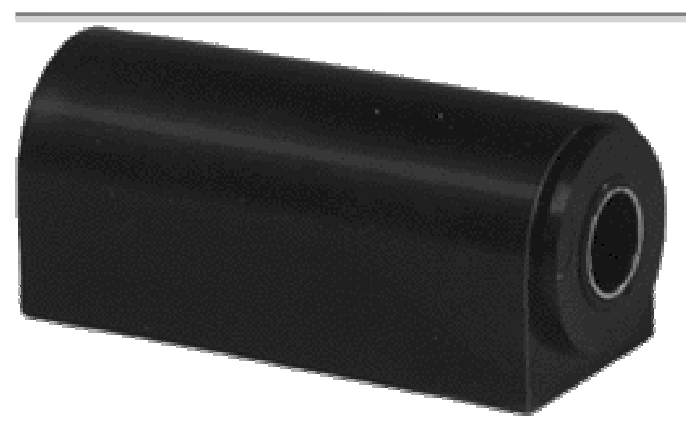

Zero Gauss Chamber: (Model ZG-1) A mu-metal shield used to shunt the earth's field around the Hall element in order to more accurately zero the gaussmeter when precise low field measurements are required.

Reference Magnets: Transverse and axial precision reference magnets are available when precise instrument calibration at a particular field is desirable. 
Threshold Control Unit: Automatic production line requirements necessitate the addition of an external control circuit which is activated when a specifically set gauss level has not been reached or when a level has been exceeded. Two "C" type, dry relay contact stacks are provided with each unit in order to accommodate two separate levels of control. Several TCU's can be used if more than two circuits are required.

Field Service Carrying Case

Specifications:

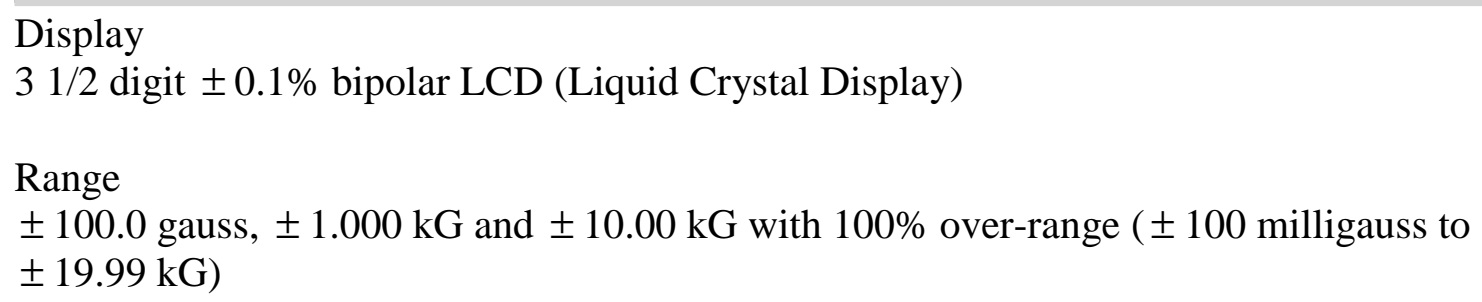

Display

$31 / 2$ digit $\pm 0.1 \%$ bipolar LCD (Liquid Crystal Display)

Range

\pm 100.0 gauss, $\pm 1.000 \mathrm{kG}$ and $\pm 10.00 \mathrm{kG}$ with $100 \%$ over-range ( \pm 100 milligauss to $\pm 19.99 \mathrm{kG})$

Resolution:

$0.05 \%$ ( \pm 100 milligauss -100.0 gauss range, \pm 1 gauss $-1.000 \mathrm{kG}$ range, $\pm ; 10$ gauss $10.00 \mathrm{kG}$ range)

$\begin{array}{ll}\begin{array}{ll}\text { Instrument Accuracy } \\ \text { Range Setting }\end{array} & \text { DC Accuracy FS } \\ \text { 100G-DC } & \pm 0.05 \% \\ \text { 1kG-DC } & \pm 0.05 \% \\ \text { 10kG-DC } & \pm 0.05 \\ & \text { RMS Accuracy } 1 \%-\mathrm{FS} \\ 100 \mathrm{G}-\mathrm{RMS} & \pm 2 \% 3 \mathrm{~Hz}-10 \mathrm{~Hz}, \pm 1 \% 10 \mathrm{~Hz}-20 \mathrm{kHz} \\ \text { 1kG RMS } & \pm 1 \% 3 \mathrm{~Hz}-10 \mathrm{~Hz}, \pm 0.3 \% 10 \mathrm{~Hz}-20 \mathrm{kHz} \\ \text { 10kG-RMS } & \pm 1 \% 3 \mathrm{~Hz}-10 \mathrm{HZ}, \pm 0.3 \% 10 \mathrm{HZ}-20 \mathrm{kHz}\end{array}$

Analog Output:

$( \pm 1.000 \mathrm{~V}$ with $100 \%$ over-range)

*Peak Meter Reading Accuracy:

(0 to \pm 19.99 count max.) $\pm 1 \%$ of full scale

\pm 1 count, (100G Range- $\pm 2 \%, 5 \%-100 \%$ F.S.)

*Peak Reading Resolution:

(10deg;C - 40deg;C) from DC to $20 \mathrm{kHz}$ (sine wave) minimum pulse width $50 \mathrm{msec}$ (square wave)

Range Peak Resolution

100G 1 Gauss

$1 \mathrm{kG} \quad 10$ Gauss

$10 \mathrm{kG} \quad 100$ Gauss 
*Peak Analog Output Accuracy:

(0 to $\pm 2.000 \mathrm{~V}$ max.)

$1 \%$ of full scale

Power:

AC: $100-125 \mathrm{~V} / 50-60 \mathrm{~Hz}$ or $200-240 \mathrm{~V} / 50-60 \mathrm{~Hz}$

DC: 6V Sealed lead acid rechargeable battery

Battery Life:

Exceeds 10 hours continuous operation with fully charged batteries, charge time, 12 hour maximum

Physical (Less Probe):

Size: $2.25 " \mathrm{H}$ x 8.5"W x 9.25"L - 5.7cm H x $21.6 \mathrm{~cm} \mathrm{~W} \mathrm{x} 23.5 \mathrm{~cm} \mathrm{~L}$

Net Weight: 4.75 lbs. $-2.2 \mathrm{~kg}$

Shipping Weight: 6.0 lbs. - $2.7 \mathrm{~kg}$

*MG-5DP only 
Gaussmeter Hall Probes

A wide selection of transverse and axial Hall
probes is available to meet most applications.
Accuracy of measurement is assured through
the use of pre-calibrated probes which are
calibrated in high accuracy fields standardized
by nuclear magnetic resonance techniques
which permit traceability to the *National
Institute of Standards and Technology (NIST).
Probes are available with accuracies from
$\pm 0.1 \%$ to $\pm 1.5 \%$ of reading. In addition, a
selection of 10X and 100X probes are
available to extend the measuring range of
this instrument to 150.0kG. These probes are
directly interchangeable and do not require
special calibration. All probes are quickly
self-calibrated to this instrument via a
convenient front panel control.
The transverse Hall probe measures magnetic
fields perpendicular to the axis of the probe.
Standard probe thickness ranges from a thin-
line version of .043" (1.09mm) to a
ruggedized version of .063" (1.60mm).
The axial Hall probe measures magnetic fields
parallel to the axis of the probe such as
solenoidal fields. Standard probe stem
diameters are 0.263" (6.68mm) and are
housed in a rigid plastic stem for maximum
protection for the most demanding industrial
and laboratory applications.
This instrument is supplied with a six foot
probe cable. However, additional cable length
can be ordered as an option. Assistance is
available to help in selecting the most suitable
probe for your application.
*Formerly National Bureau of Standards
(NBS)




\begin{tabular}{|c|c|c|c|c|c|c|}
\hline \multicolumn{7}{|c|}{ Transverse Hall Probes } \\
\hline Model & $\begin{array}{l}\text { \% Linearity of } \\
\text { Reading }\end{array}$ & $\begin{array}{l}\text { Temperature } \\
\text { Coefficient } \\
\% / \& \text { deg;C }\end{array}$ & $\begin{array}{l}\text { Temperature } \\
\text { Range \&deg;C }\end{array}$ & Style & $\begin{array}{l}\text { Stem Size }(L \times \text { X x T) } \\
\text { or }(L \times D)\end{array}$ & $\begin{array}{l}\text { Active Area } \\
(\mathrm{L} \times \mathrm{x} \text { ) }\end{array}$ \\
\hline HP-14S & $\pm 1 \%$ to $20 \mathrm{kG}$ & -0.1 & -55 to +100 & $\mathrm{~V}-1 \mathrm{X}$ & $\begin{array}{l}0.5 "(12.70 \mathrm{~mm}) 0.025 " \\
(6.35 \mathrm{~mm})\end{array}$ & $.040 " x .080 "(1 \times 2 \mathrm{~mm})$ \\
\hline $\begin{array}{l}\text { HP-145S } \\
\text { HP-145F } \\
\text { HP-145R }\end{array}$ & $\pm 1 \%$ to $20 \mathrm{kG}$ & -0.1 & -55 to +100 & $\begin{array}{l}\mathrm{I}-1 \mathrm{X} \\
\mathrm{II}-1 \mathrm{X} \\
\mathrm{I}-1 \mathrm{X}\end{array}$ & $\begin{array}{l}4 "(10.16 \mathrm{~cm}) \times 0.155 "(3.94 \mathrm{~m} \\
\mathrm{m}) \mathrm{x} 0.0433^{\prime \prime}(1.09 \mathrm{~mm}) \\
4 "(10.16 \mathrm{~cm}) \times 0.165 "(4.19 \mathrm{~m} \\
\mathrm{m}) \mathrm{x} 0.053 "(1.35 \mathrm{~mm}) \\
4 "(10.16 \mathrm{~cm}) \times 0.175 "(4.45 \mathrm{~m} \\
\mathrm{m}) \mathrm{x} 0.063 "(1.60 \mathrm{~mm})\end{array}$ & $.040 " x .080 "$ (1x2mm) \\
\hline $\begin{array}{l}\text { HP-345S-(*) } \\
\text { HP-345F-(*) } \\
\text { HP-345R-(*) }\end{array}$ & $\begin{array}{l} \pm 0.25 \% \text { to } 10 \mathrm{kG}-(10) \\
\pm 0.5 \% \text { to } 20 \mathrm{kG}-(20) \\
\pm 1 \% \text { to } 30 \mathrm{kG}-(30)\end{array}$ & -0.04 & -40 to +100 & $\begin{array}{l}\text { I-10X } \\
\text { II-10X } \\
\text { I-10X }\end{array}$ & $\begin{array}{l}4 "(10.16 \mathrm{~cm}) \times 0.155 "(3.94 \mathrm{~m} \\
\text { m)x0.043"(1.09mm) } \\
4 "(10.16 \mathrm{~cm}) \times 0.165 "(4.19 \mathrm{~m} \\
\text { m)x0.053"(1.35mm) } \\
4 "(10.16 \mathrm{~cm}) \times 0.175 "(4.45 \mathrm{~m} \\
\text { m)x0.063"(1.60mm }) \\
\end{array}$ & $.040 "$ Dia. (1mm) \\
\hline $\begin{array}{l}\text { HP-645S } \\
\text { HP-645F } \\
\text { HP-645R }\end{array}$ & $\pm 0.1 \%$ to $30 \mathrm{kG}$ & \pm 0.005 & -40 to +100 & $\begin{array}{l}\text { I-100X } \\
\text { II-100X } \\
\text { I-100X }\end{array}$ & $\begin{array}{l}4 "(10.16 \mathrm{~cm}) \times 0.155 "(3.94 \mathrm{~m} \\
\mathrm{m}) \mathrm{x} 0.043 "(1.09 \mathrm{~mm}) \\
44^{\prime \prime}(10.16 \mathrm{~cm}) \times 0.165 "(4.19 \mathrm{~m} \\
\mathrm{m}) \mathrm{x} 0.053 "(1.35 \mathrm{~mm}) \\
4^{\prime \prime}(10.16 \mathrm{~cm}) \times 0.175 "(4.45 \mathrm{~m} \\
\mathrm{m}) \mathrm{x} 0.063 "(1.60 \mathrm{~mm})\end{array}$ & $.040 "$ Dia. (1mm) \\
\hline $\begin{array}{l}\mathrm{HP}-1145 \mathrm{~S} \\
\mathrm{HP}-1145 \mathrm{~F} \\
\mathrm{HP}-1145 \mathrm{R}\end{array}$ & $\begin{array}{l} \pm 1 \% \text { to } 30 \mathrm{kG} / \\
\pm 1.5 \% \text { to } 150 \mathrm{kG}\end{array}$ & \pm 0.005 & -40 to +100 & $\begin{array}{l}\mathrm{I}-100 \mathrm{X} \\
\mathrm{II}-100 \mathrm{X} \\
\mathrm{I}-100 \mathrm{X}\end{array}$ & $\begin{array}{l}4 "(10.16 \mathrm{~cm}) \times 0.155 "(3.94 \mathrm{~m} \\
\mathrm{m}) \times 0.043 "(1.09 \mathrm{~mm}) \\
4 "(10.16 \mathrm{~cm}) \times 0.165 "(4.19 \mathrm{~m} \\
\mathrm{m}) \mathrm{x} 0.053 "(1.35 \mathrm{~mm}) \\
4 "(10.16 \mathrm{~cm}) \times 0.175 "(4.45 \mathrm{~m} \\
\mathrm{m}) \times 0.063 "(1.60 \mathrm{~mm}) \\
\end{array}$ & $.040 "$ Dia. (1mm) \\
\hline \multicolumn{7}{|c|}{ Axial Hall Probes } \\
\hline HP-24S & $\pm 1 \%$ to $30 \mathrm{kG}$ & -0.1 & -55 to +100 & $\mathrm{~V}-1 \mathrm{X}$ & \begin{tabular}{|l}
$0.5 "(12.70 \mathrm{~mm})$ \\
$0.250 "(6.35 \mathrm{~mm})$
\end{tabular} & \begin{tabular}{|l}
$.040 " x .080 "$ \\
$(1 \mathrm{x} 2 \mathrm{~mm})$
\end{tabular} \\
\hline $\begin{array}{l}\mathrm{HP}-245 \mathrm{~S} \\
\mathrm{HP}-245 \mathrm{~F}\end{array}$ & $\pm 1 \%$ to $20 \mathrm{kG}$ & -0.1 & -55 to +100 & \begin{tabular}{|l|} 
III-1X \\
IV-1X
\end{tabular} & $\begin{array}{l}.0 "(12.70 \mathrm{~cm}) \\
0.263 "(6.68 \mathrm{~mm})\end{array}$ & $\begin{array}{l}.040 " \mathrm{x} .080 " \\
(1 \mathrm{x} 2 \mathrm{~mm})\end{array}$ \\
\hline $\begin{array}{l}\text { HP-845S-(*) } \\
\text { HP-845F-(*) }\end{array}$ & $\begin{array}{l} \pm 0.25 \% \text { to } 10 \mathrm{kG}- \\
(10) \\
\pm 0.5 \% \text { to } 20 \mathrm{kG}- \\
(20) \\
\pm 1 \% \text { to } 30 \mathrm{kG}-(30)\end{array}$ & -0.04 & -40 to +100 & $\begin{array}{l}\text { III-10X } \\
\text { IV-10X }\end{array}$ & $\begin{array}{l}9.0^{\prime \prime}(22.86 \mathrm{~cm}) \\
0.263 "(6.68 \mathrm{~mm})\end{array}$ & $.040 "$ Dia. $(1 \mathrm{~mm})$ \\
\hline $\begin{array}{l}\text { HP-1045S } \\
\text { HP-1045F }\end{array}$ & $\pm 0.25 \%$ to $30 \mathrm{kG}$ & \pm 0.005 & -40 to +100 & \begin{tabular}{|l|} 
III-100X \\
IV-100X \\
\end{tabular} & $\begin{array}{l}9.0 "(22.86 \mathrm{~cm}) \\
0.263 "(6.68 \mathrm{~mm})\end{array}$ & $.020 "$ Dia. $(0.5 \mathrm{~mm})$ \\
\hline \begin{tabular}{|l} 
HP-1245S \\
HP-1245F
\end{tabular} & $\begin{array}{l} \pm 1 \% \text { to } 30 \mathrm{kG} / \\
\pm 1.5 \% \text { to } 150 \mathrm{kG}\end{array}$ & \pm 0.005 & -40 to +100 & \begin{tabular}{|l|} 
III-100X \\
IV-100X
\end{tabular} & \begin{tabular}{|l}
$9.0 "(22.86 \mathrm{~cm})$ \\
$0.263 "(6.68 \mathrm{~mm})$
\end{tabular} & $.020 "$ Dia. $(0.5 \mathrm{~mm})$ \\
\hline
\end{tabular}

* - Specify

Model Number Suffix: S-Standard, F-Flexible, R-Ruggedized

Note: Thickness of standard probe applies to $1 / 2$ " from tip only.

The above listing is representative of our most widely used probes. Please consult us if your application requirements cannot be met by one of these probes. 
Appendix 3 - Data Acquisition System Specifications

DI-151RS Starter Kit

\section{Product Highlights}

Low cost, Compact Data Acquisition Kit

Record Data With 12-bits of Measurement

Accuracy at Rates up to 240 Samples/sec

Convenient Serial Port Interface

Two +/- $10 \mathrm{~V}$ analog inputs

Two Digital inputs for remote Start/Stop

and event markers

Thermistor inputs

Includes WINDAQ Software,

Communications Cable, and

Documentation

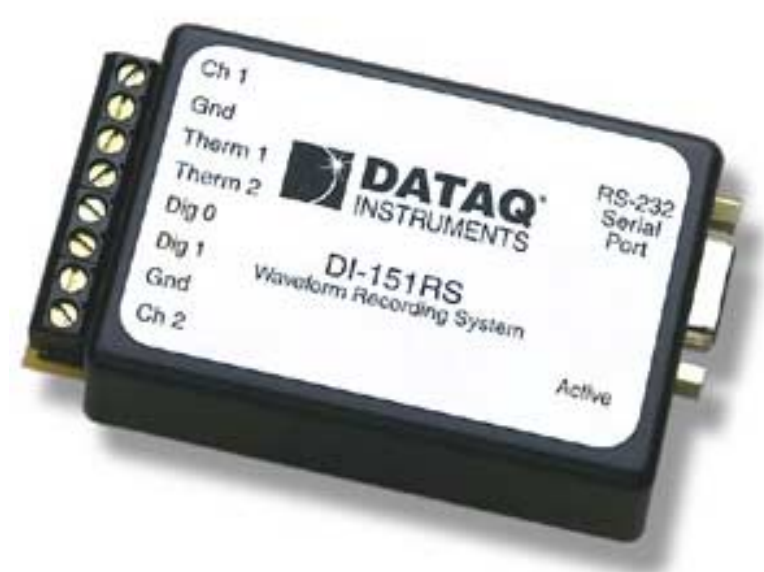

The DI-151RS Starter Kit is still a low cost way to familiarize you with WINDAQ software, but it has more features and capabilities than ever before. With the DI-151RS, you can digitize virtually any transducer's analog output signal and record it to your PC's hard disk. At the same time, view the transducer's output on your PC's monitor in a triggered sweep (oscilloscope-like) or scrolling (chart recorder-like) display format. ThisDI-151RS Starter Kit is the same serial port waveform recording and analysis system being sold by Radio Shack® in their current catalog.

Self-Powered Advantage

The DI-151RS derives its power directly from the RS-232 serial port line to which it is connected - no batteries to replace or external power supplies to connect.

More Capabilities

The DI-151RS is equipped with two dedicated thermistor (Part \#: DC104R2K, 100K Ohm @ 25C, U.S. SENSORS, Tel: 714-639-1000)inputs. Two +/- 10 volt analog inputs for connecting to your high level signals. Two digital inputs for remote start/stop and event markers.

WinDaq Software Included

The DI-151RS Starter Kit includes WINDAQ/Lite recording software and WINDAQ Waveform Browser playback and analysis software. 
Appendix 4 - Magnet Specifications \& Drawings

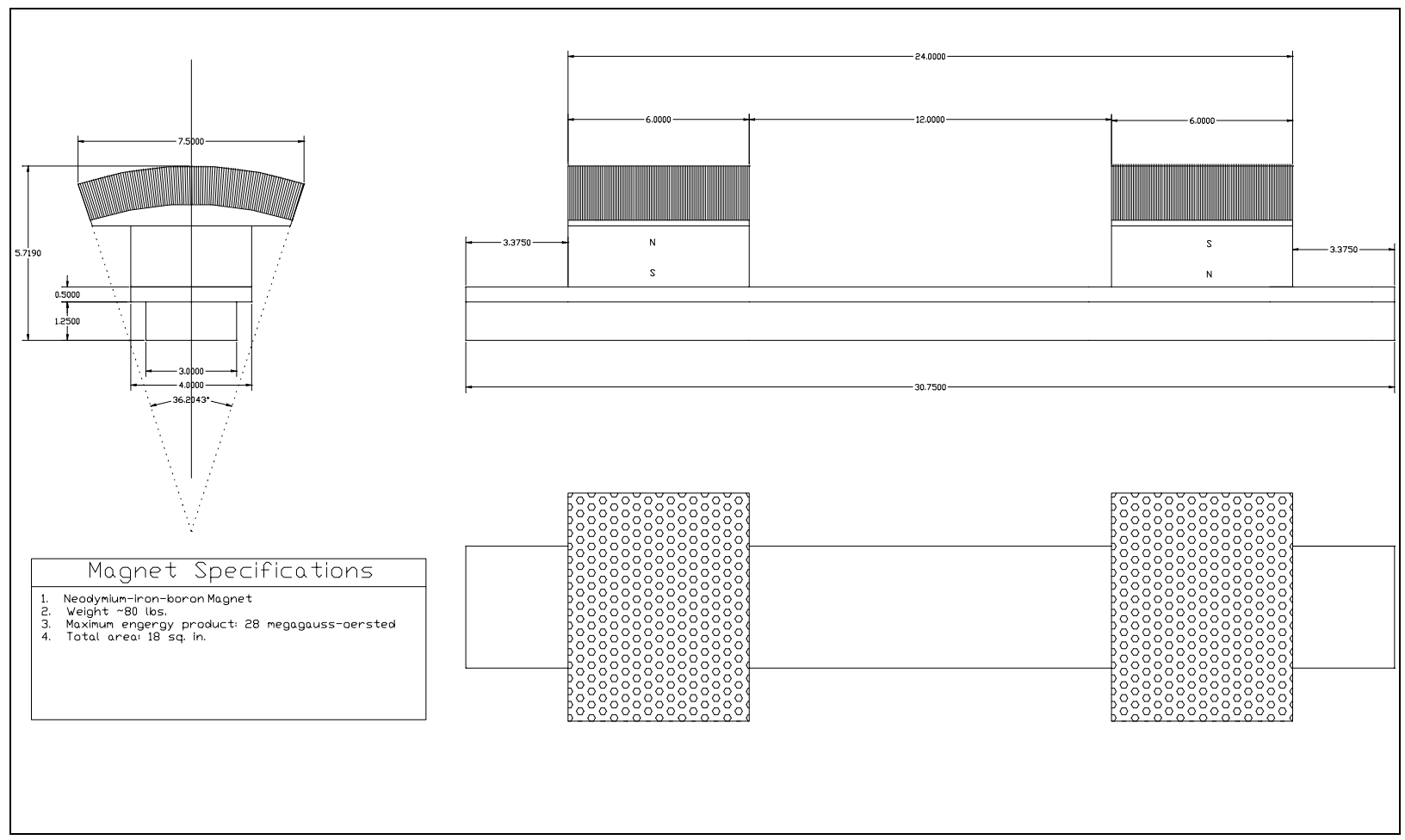


Appendix 5 - Pipe coupon specifications \& drawings

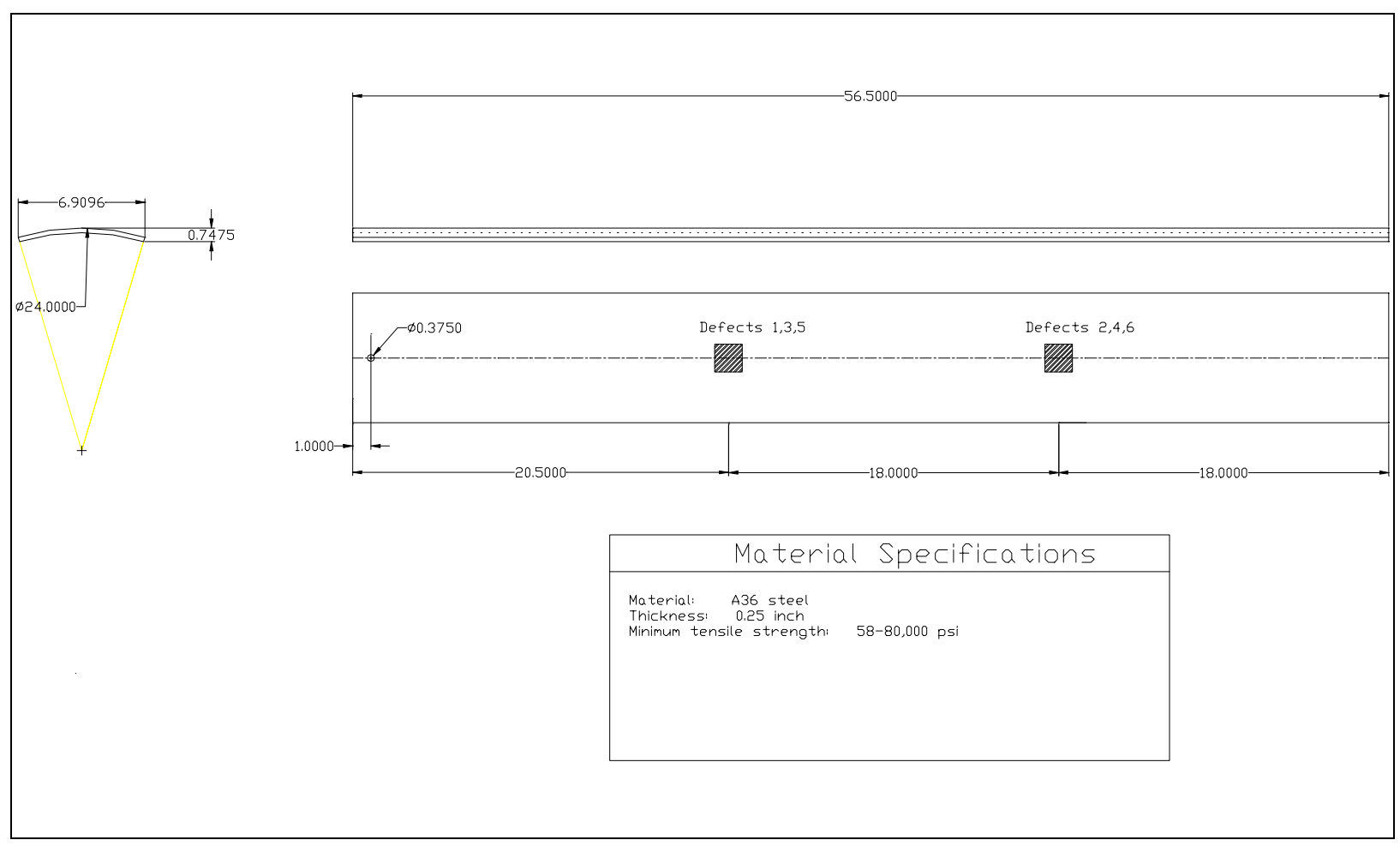


Appendix 6 - Defect dimensions

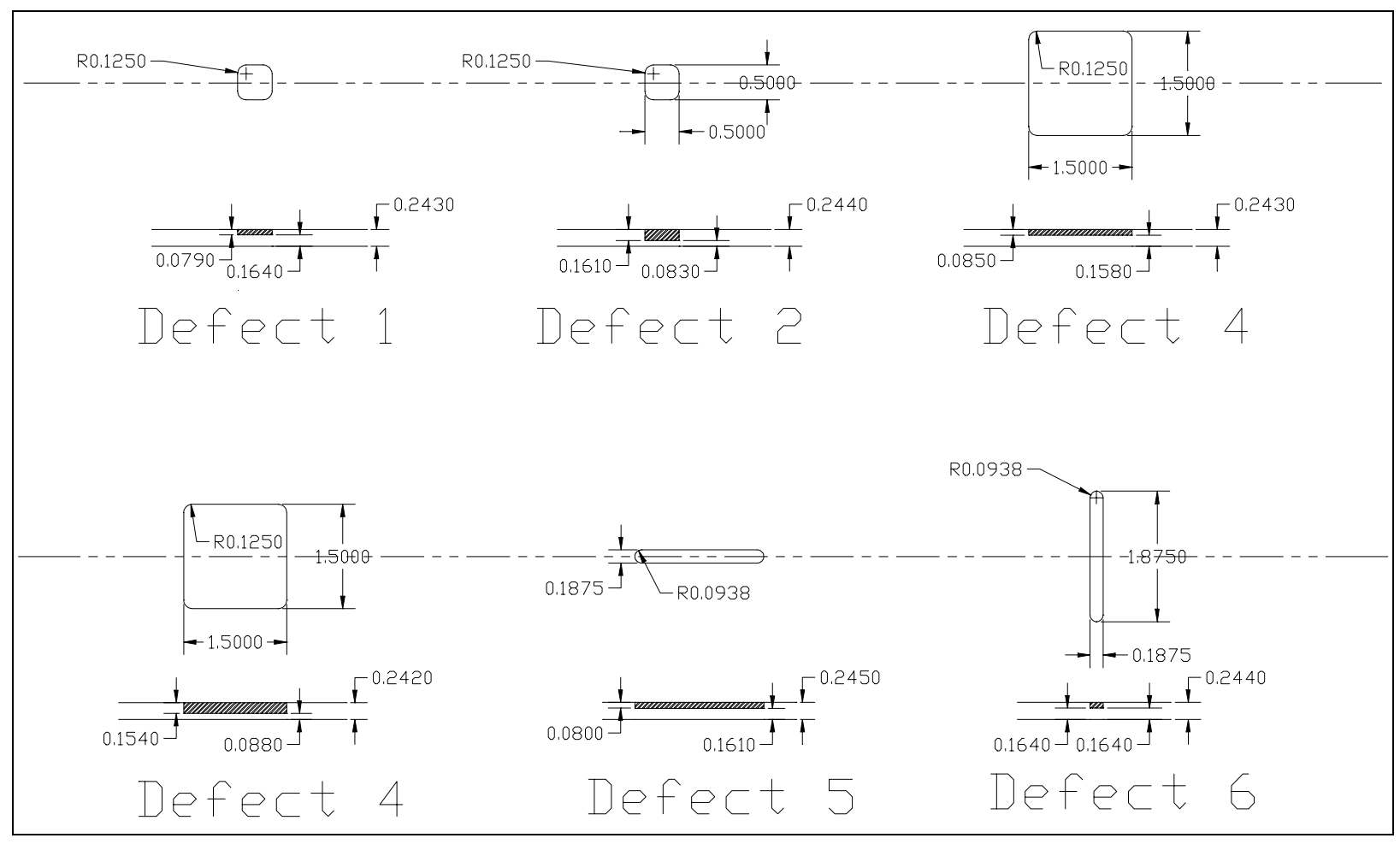


Appendix 7 - Sensor holder specifications \& drawings

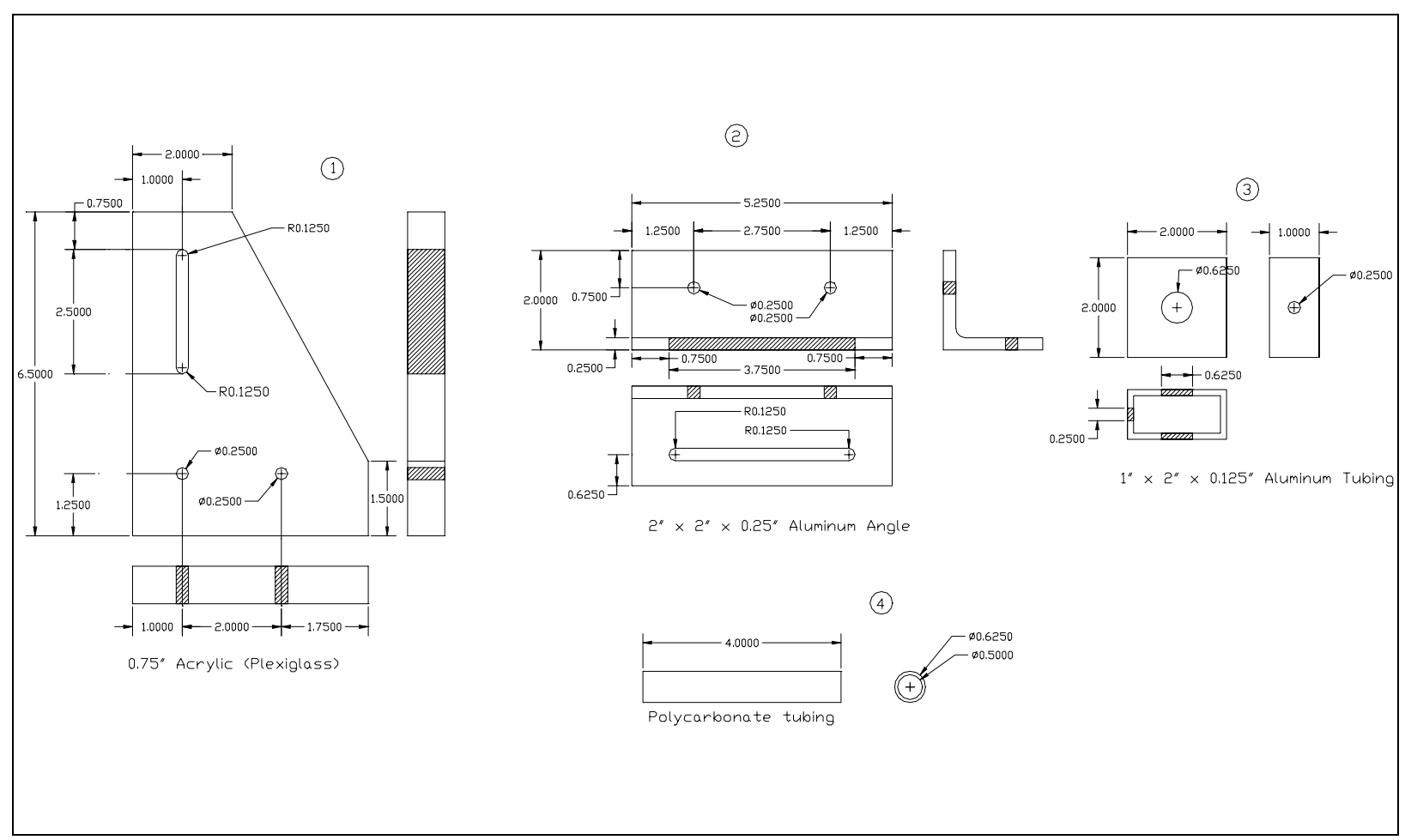


Appendix 8 - Coupon guides specifications \& drawings

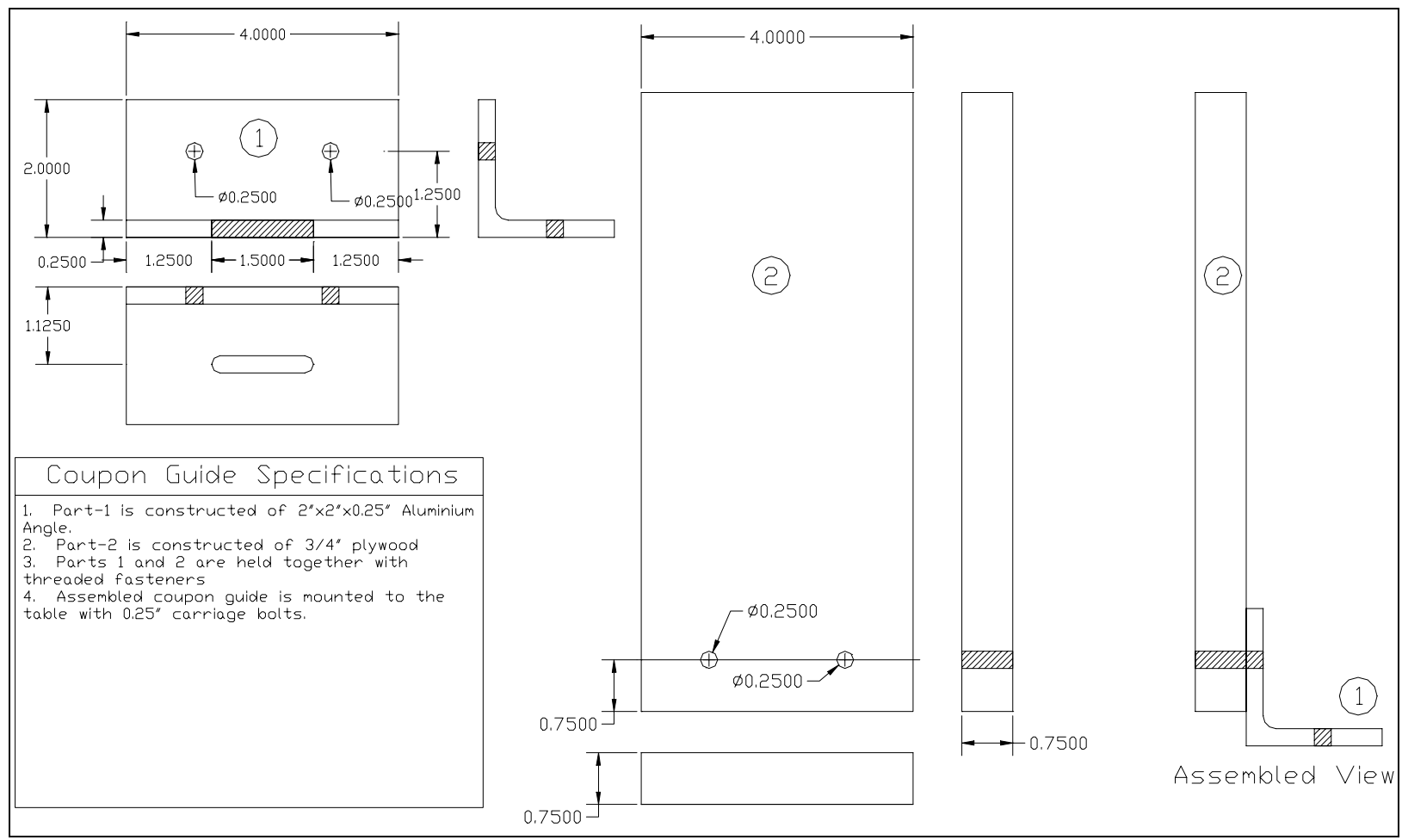


Appendix 9 - Displacement device specifications \& drawings

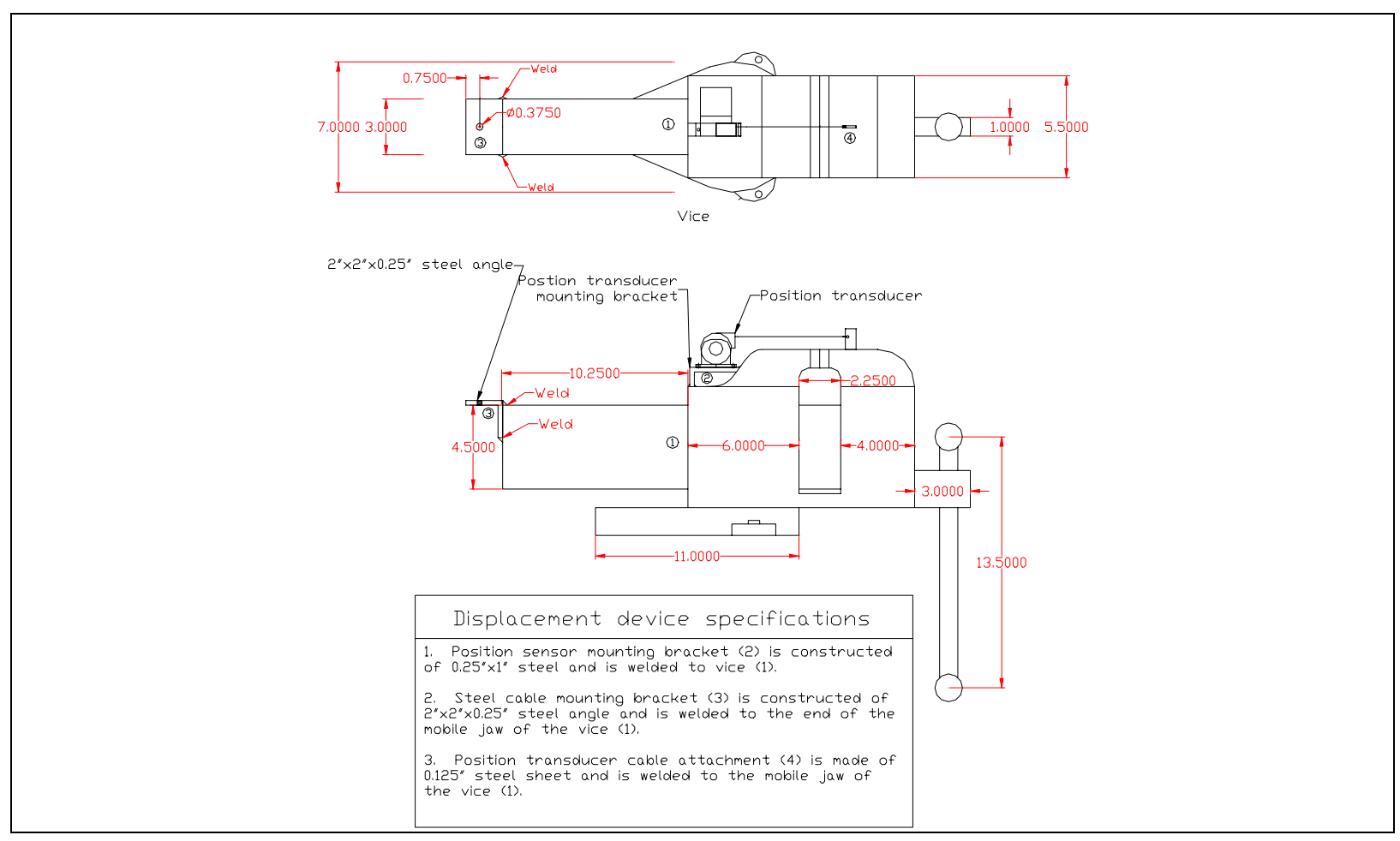


Appendix 10 - Magnet lift-off device specifications \& drawings

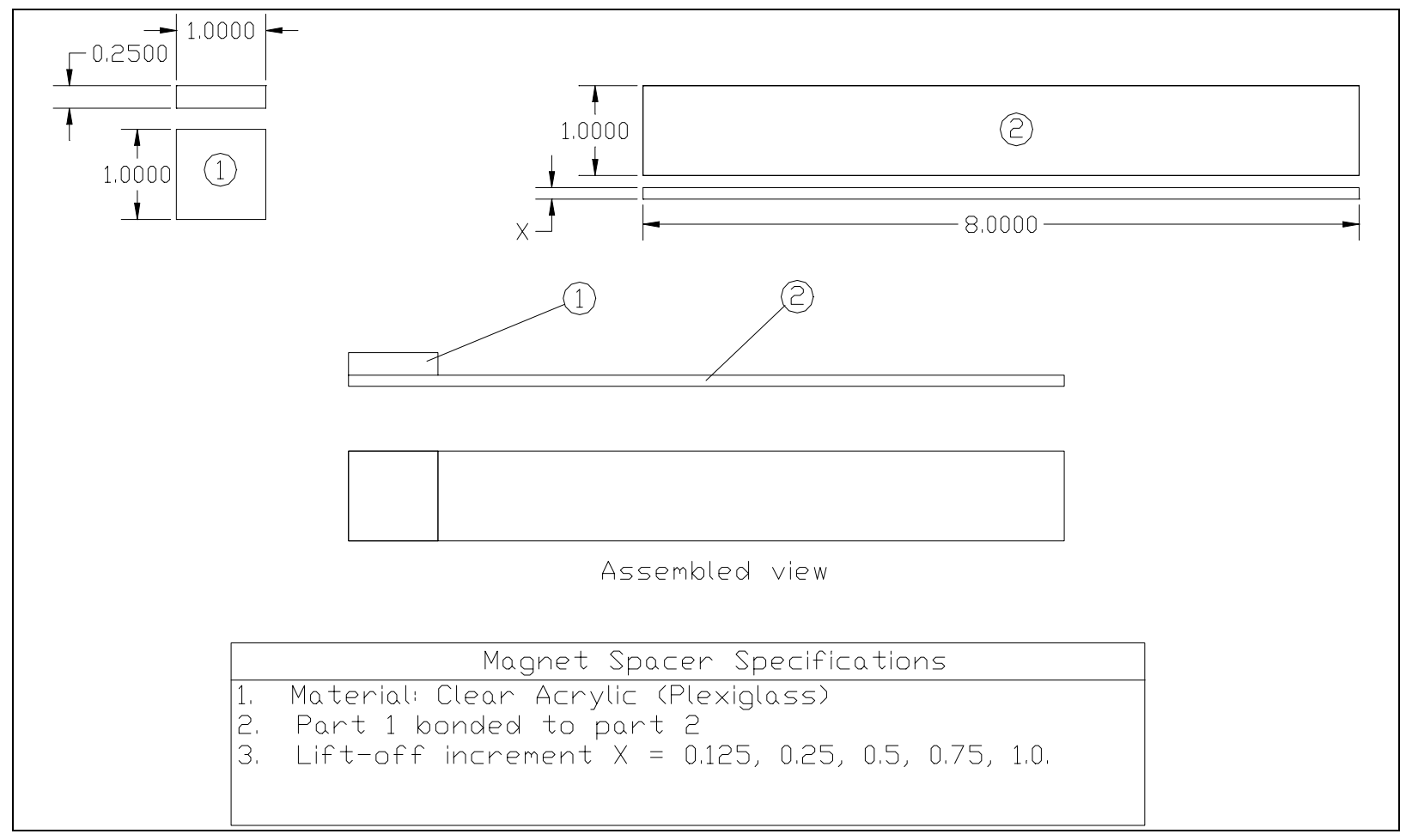


Appendix 11 - Sensor lift-off graphs (zero magnetizer lift-off)
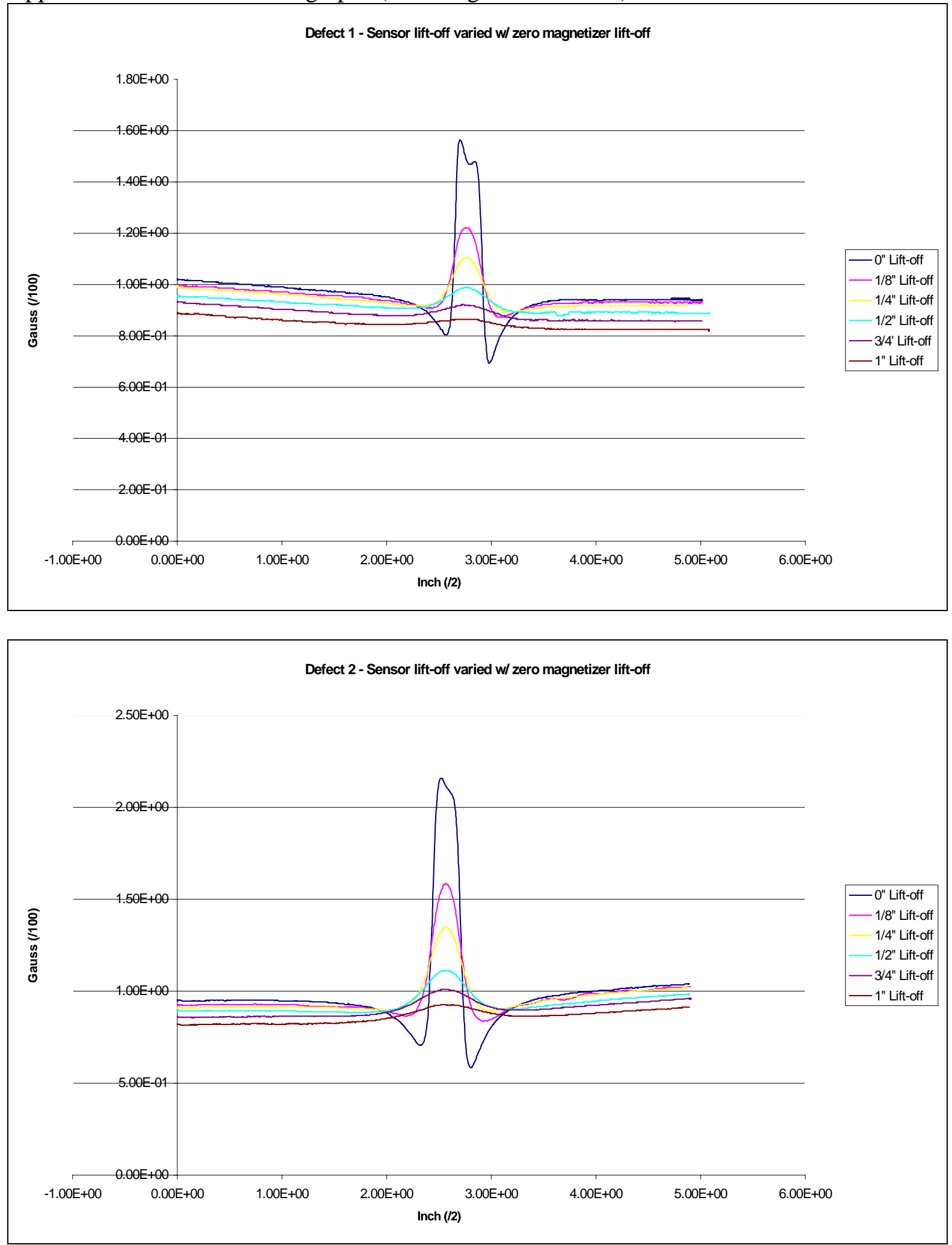

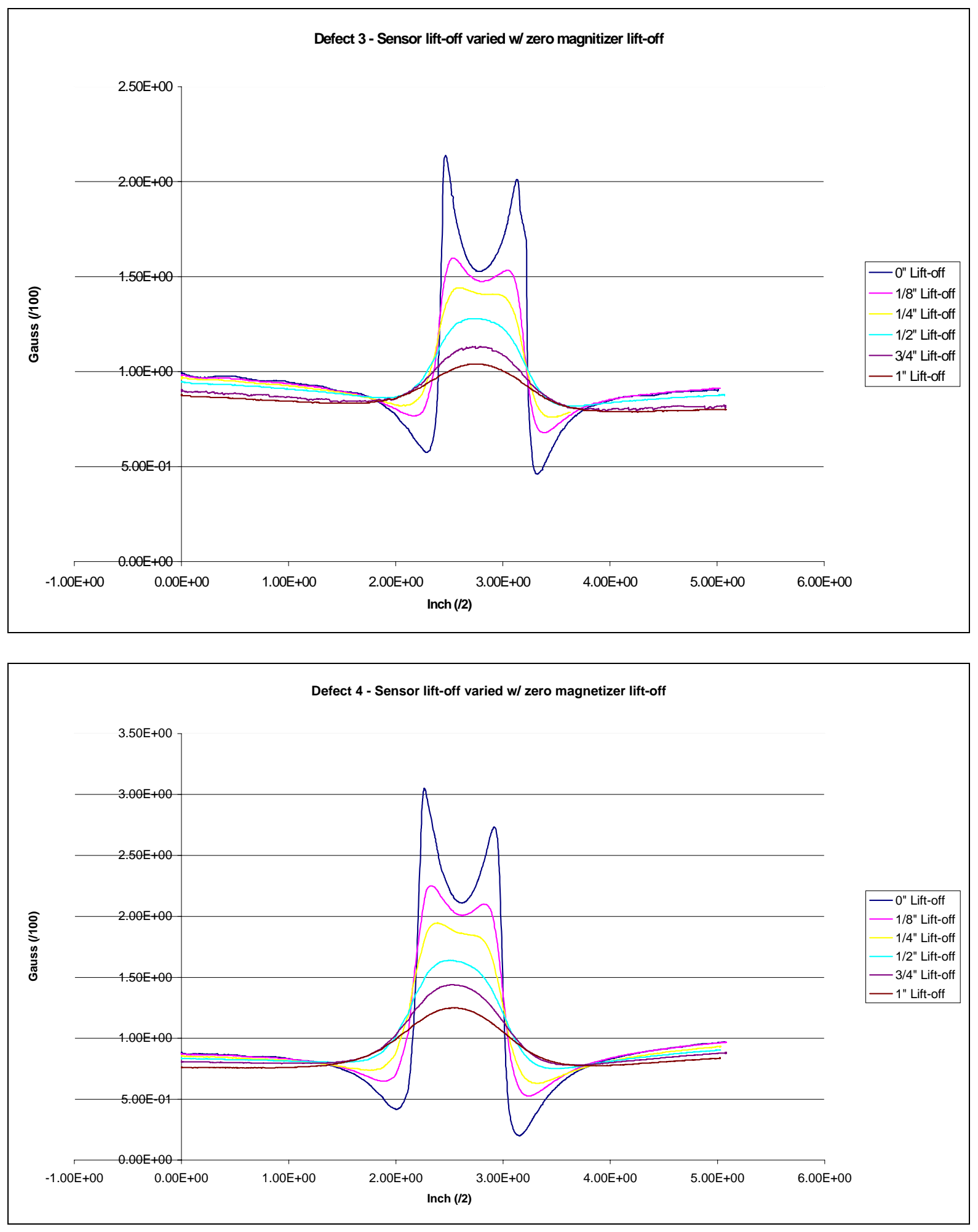

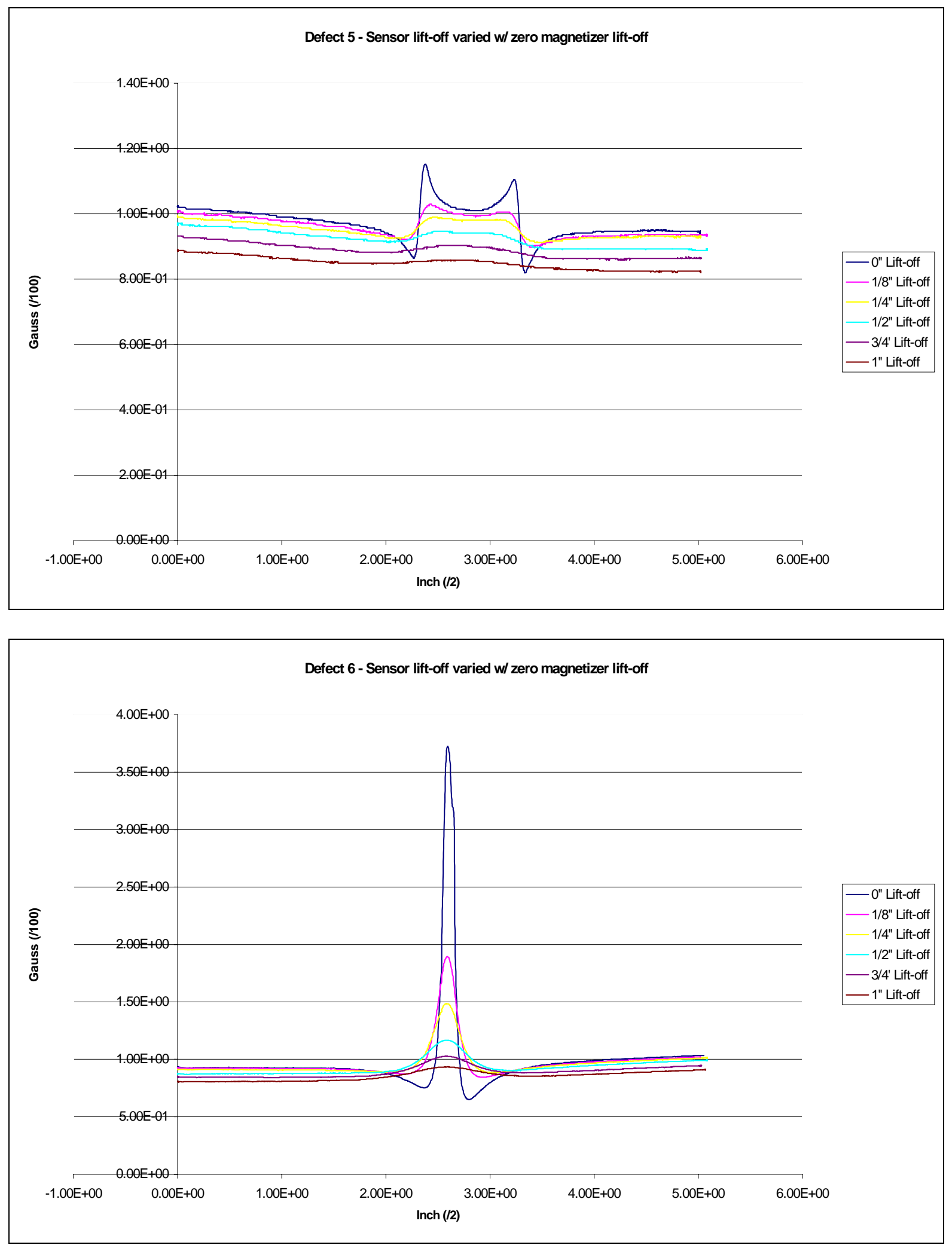
Appendix 12 - Magnetizer lift-off graphs (zero sensor lift-off)
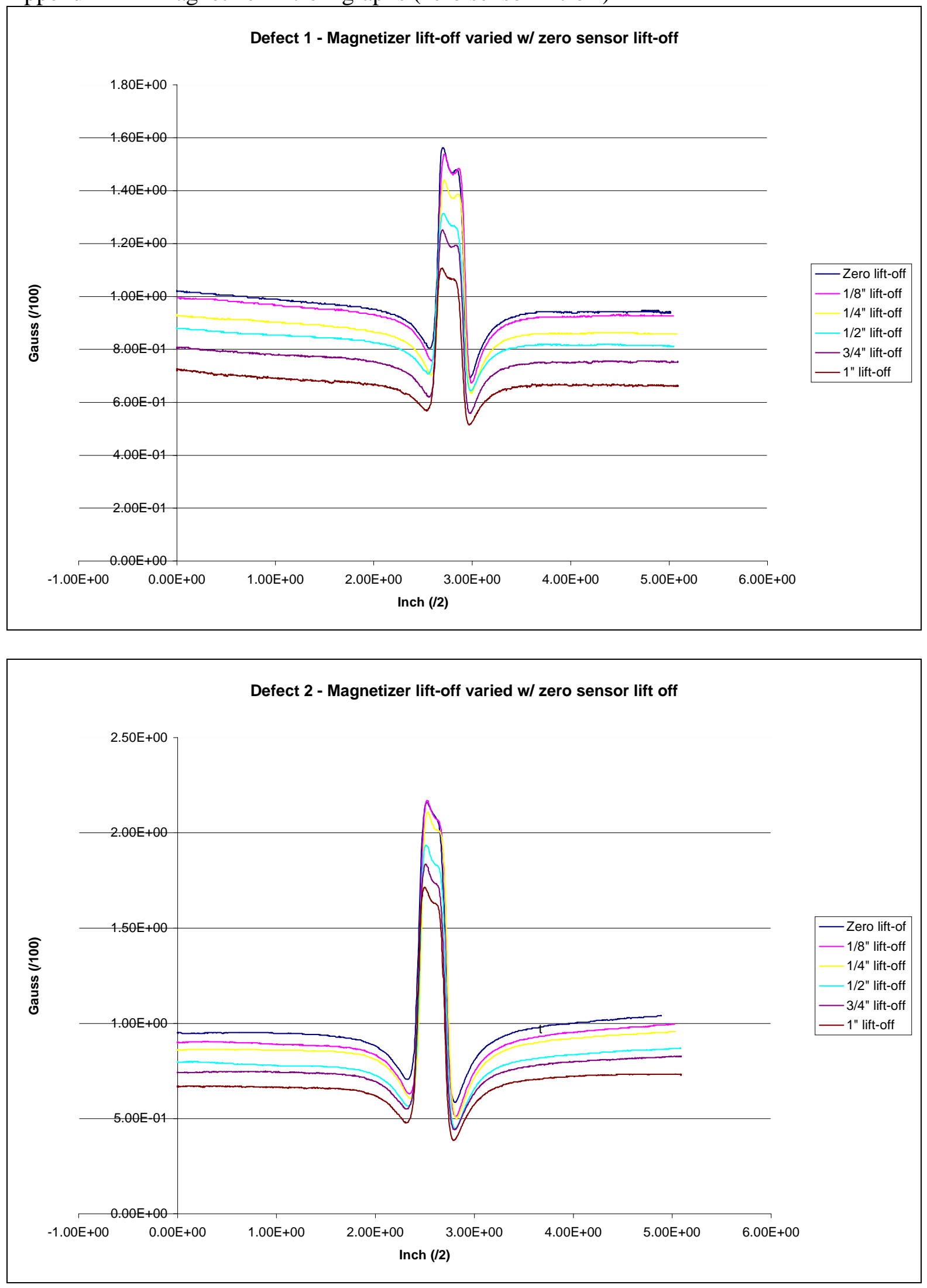

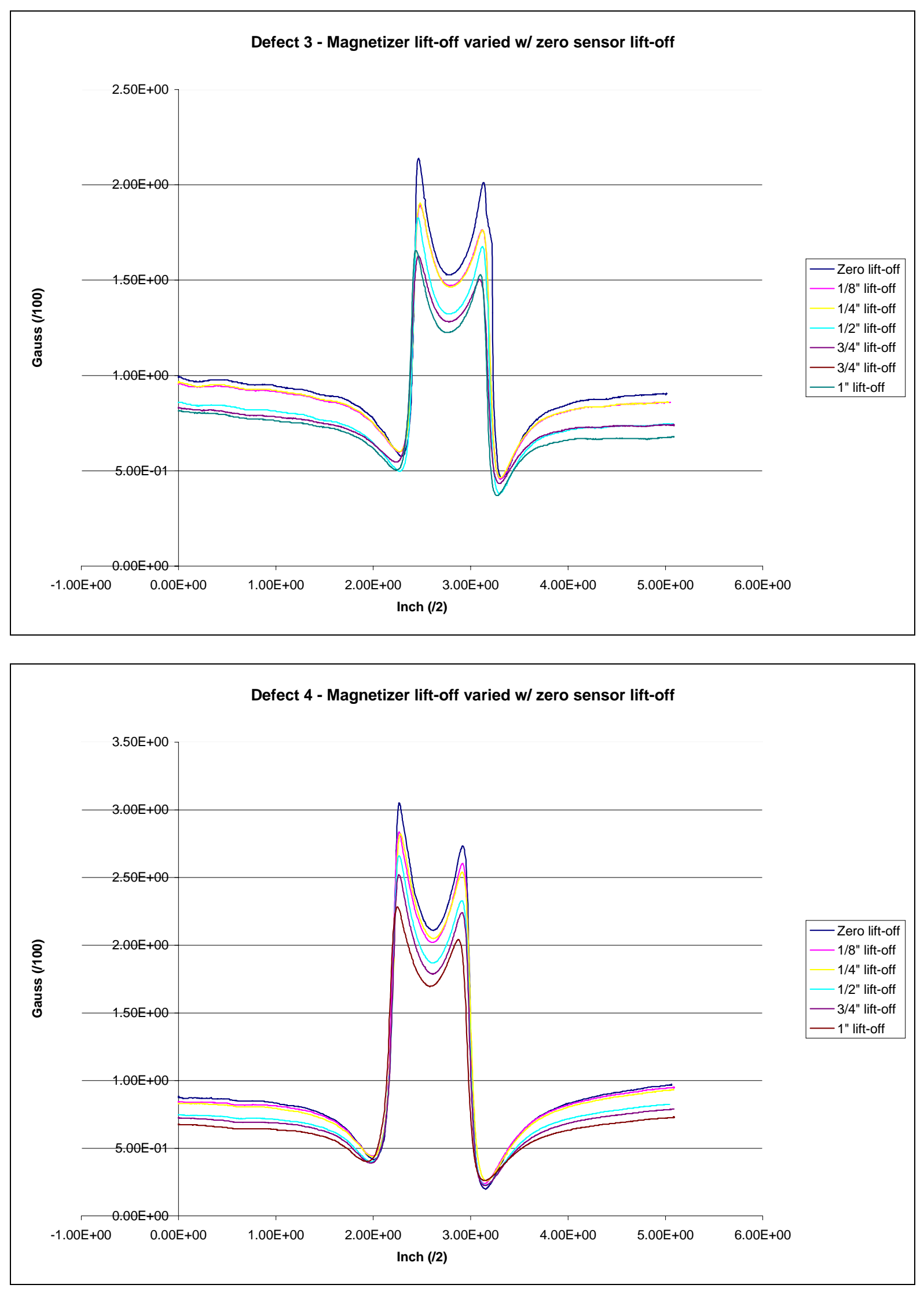

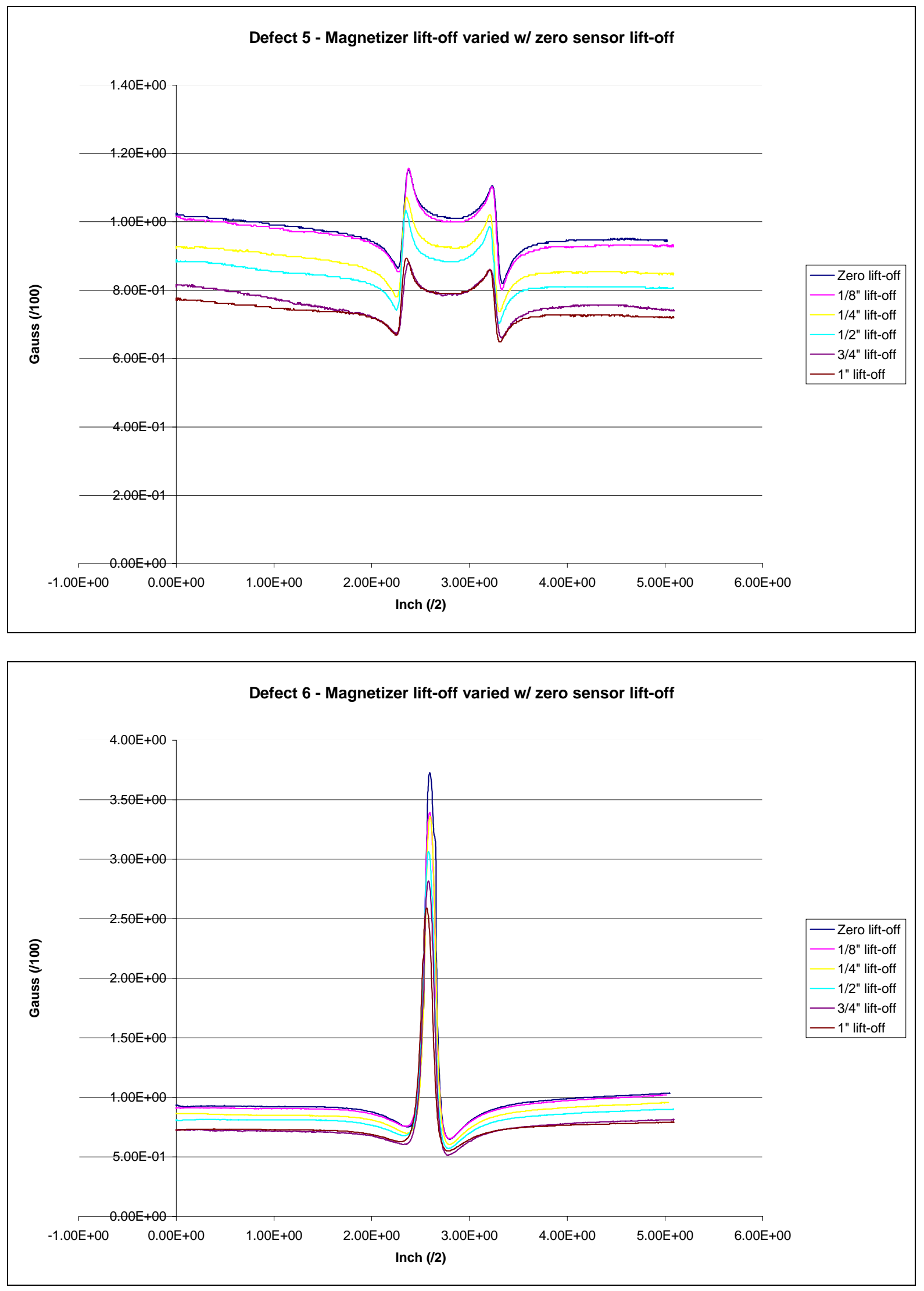
Appendix 13 - Simultaneous sensor and magnetizer lift-off graphs
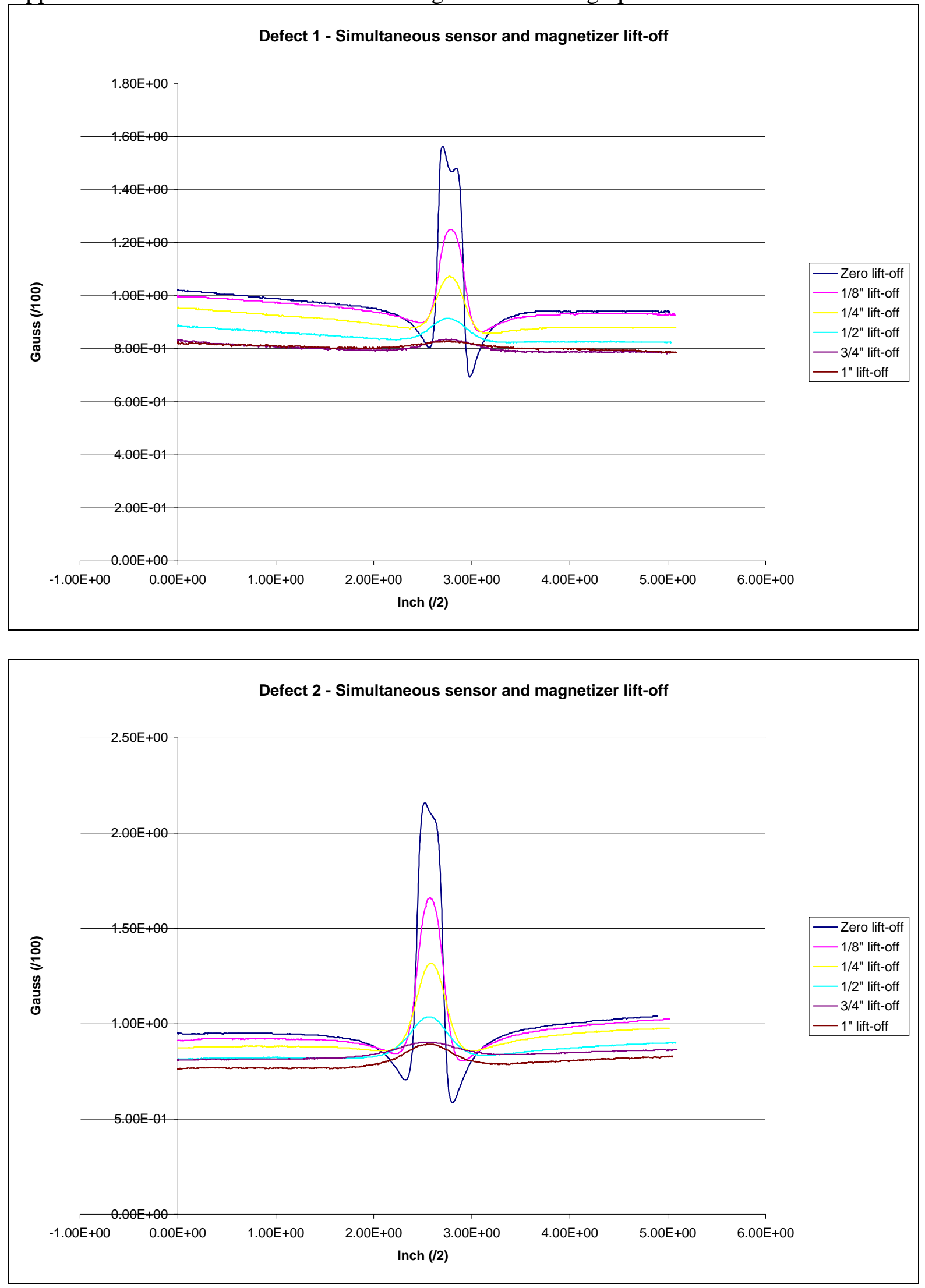

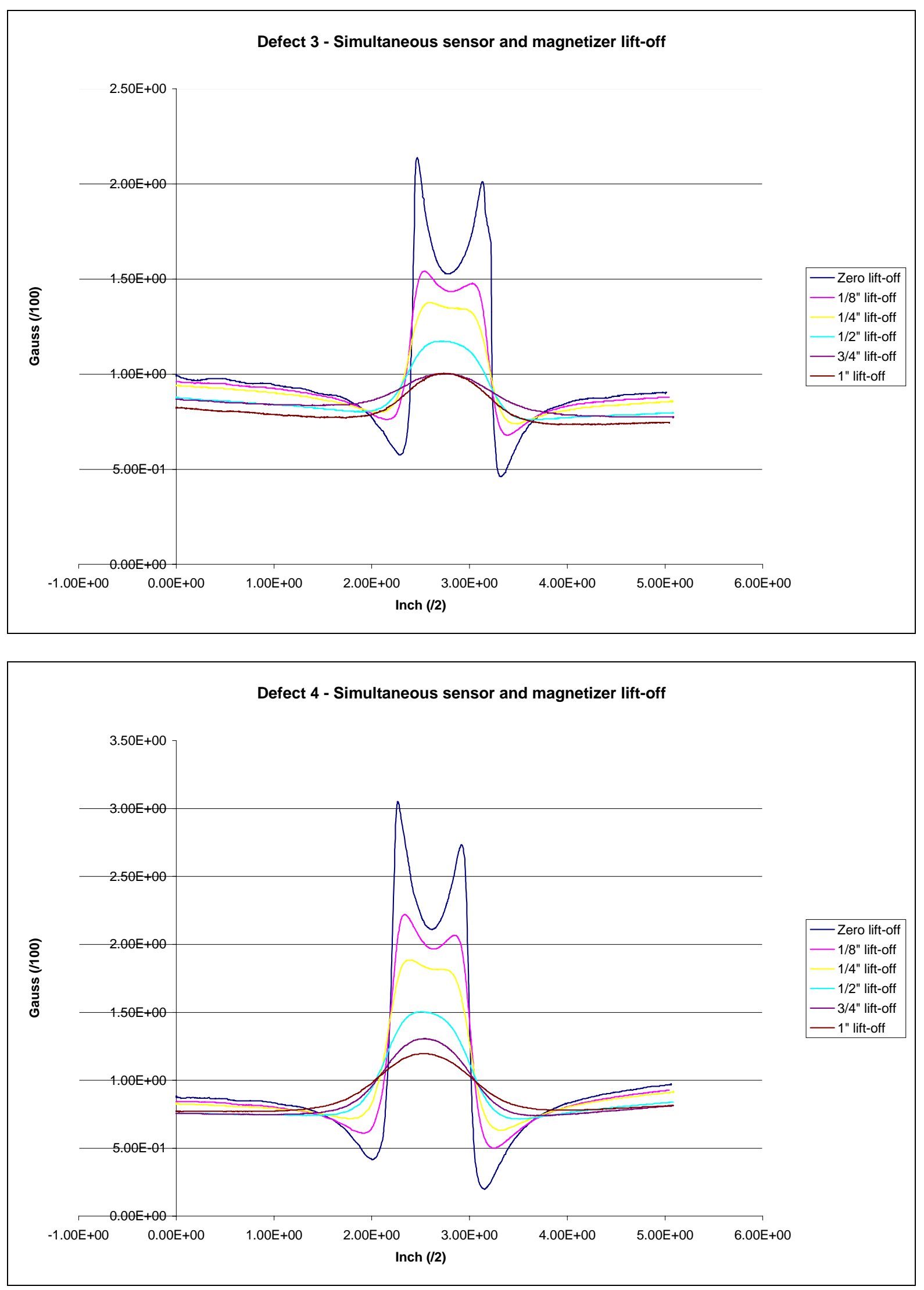

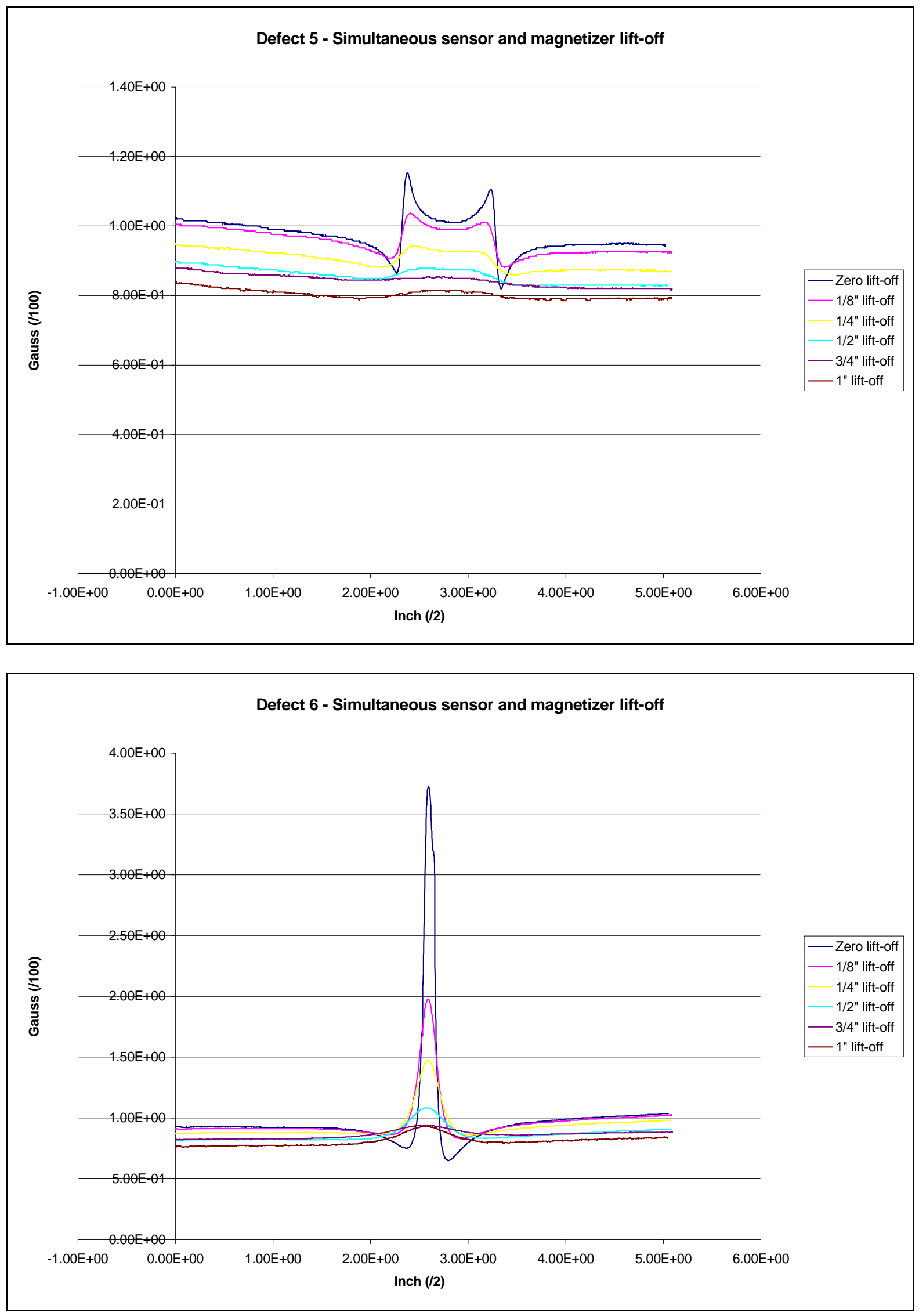
Appendix 14 - Defect 1 - Magnetic flux leakage signal comparison graphs
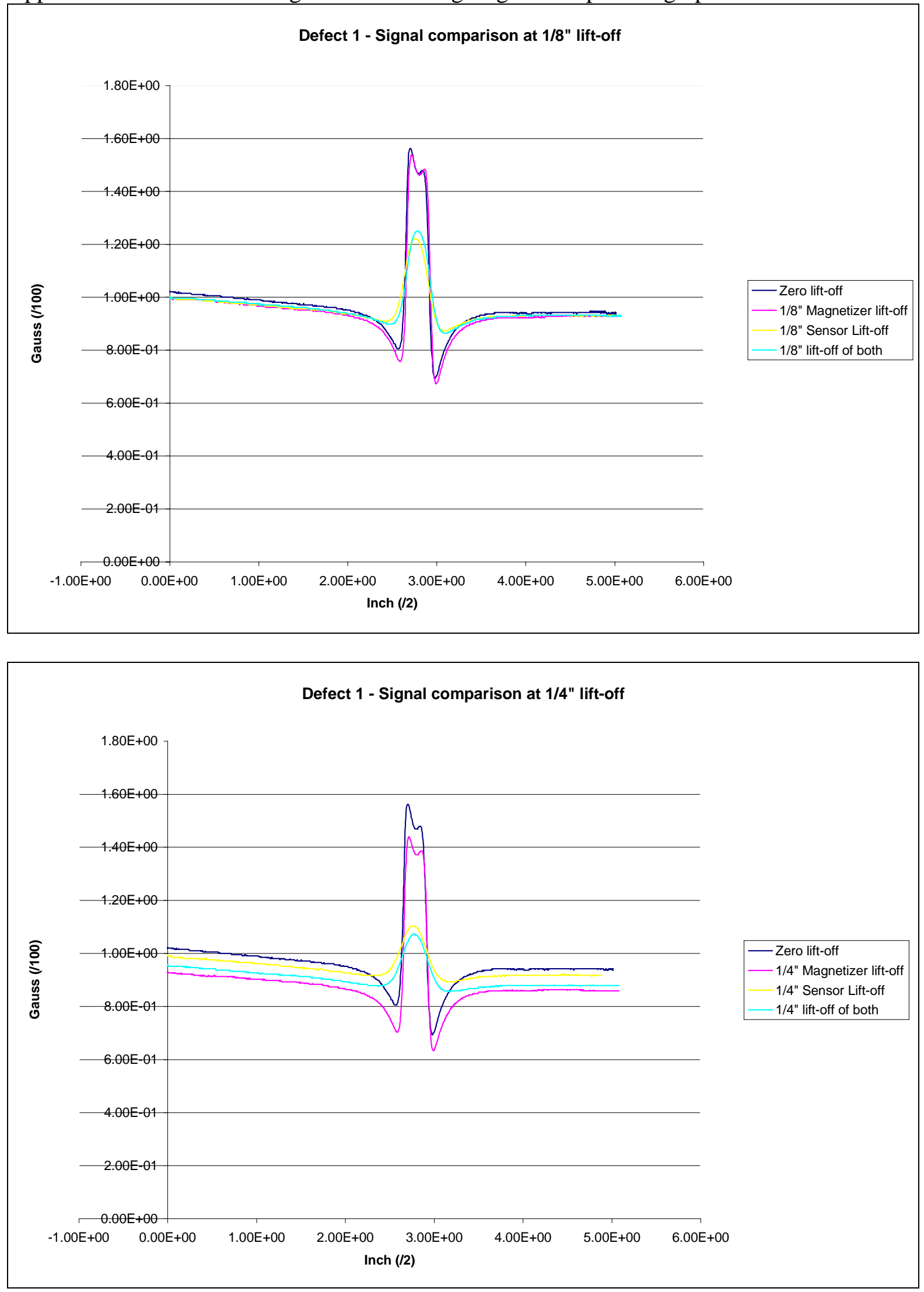

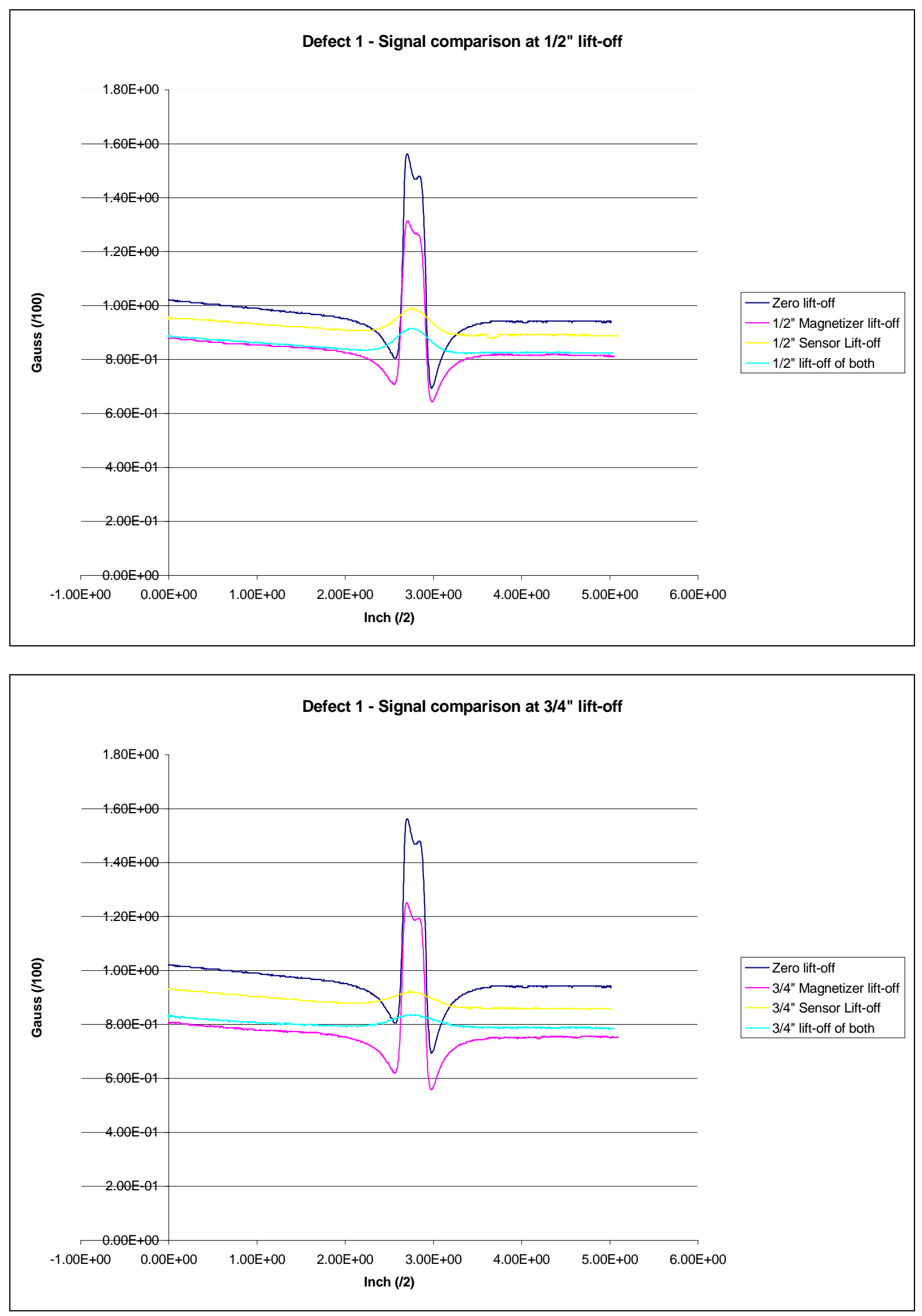


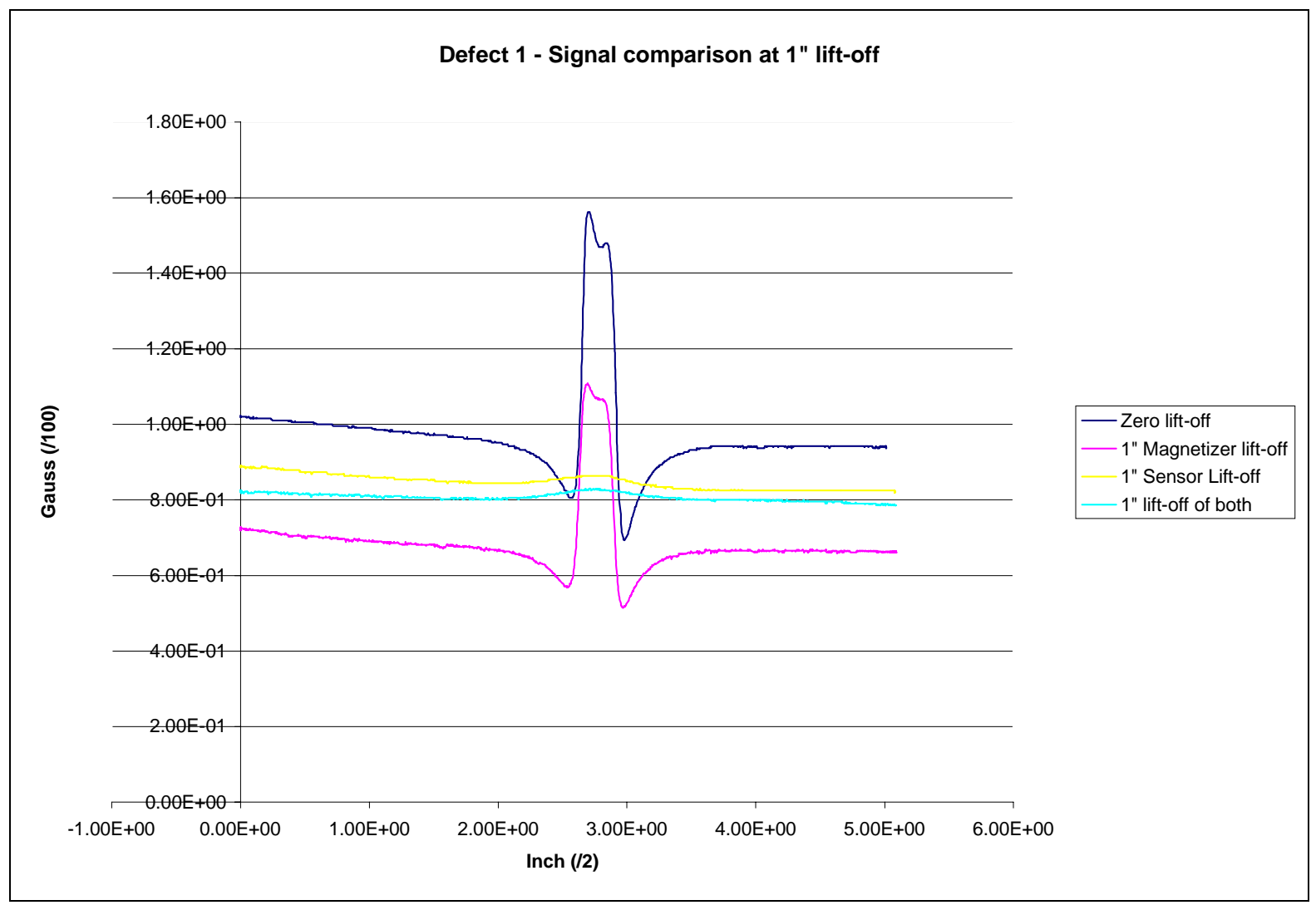


Appendix 15 - Defect 2 - Magnetic flux leakage signal comparison graphs
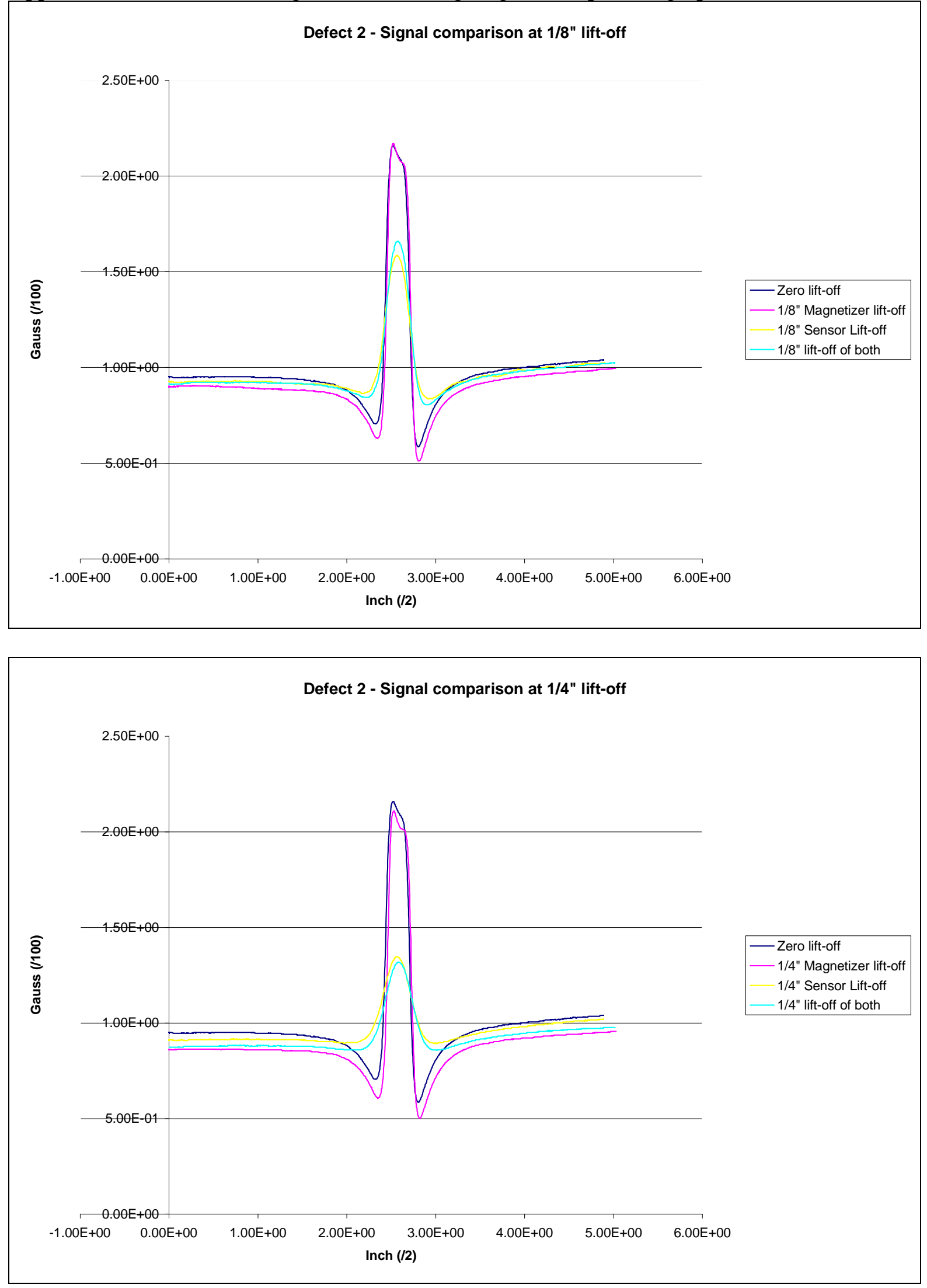

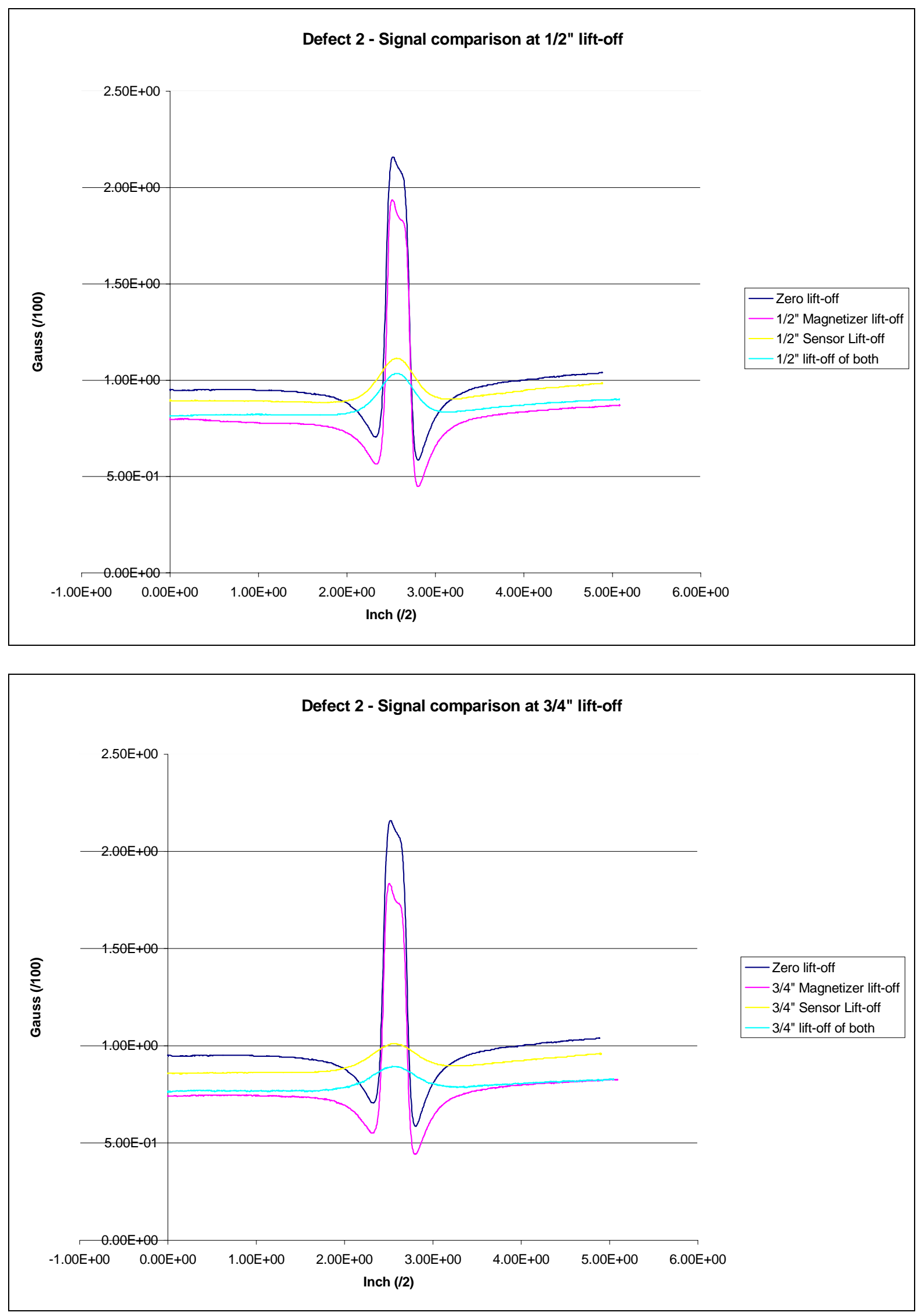


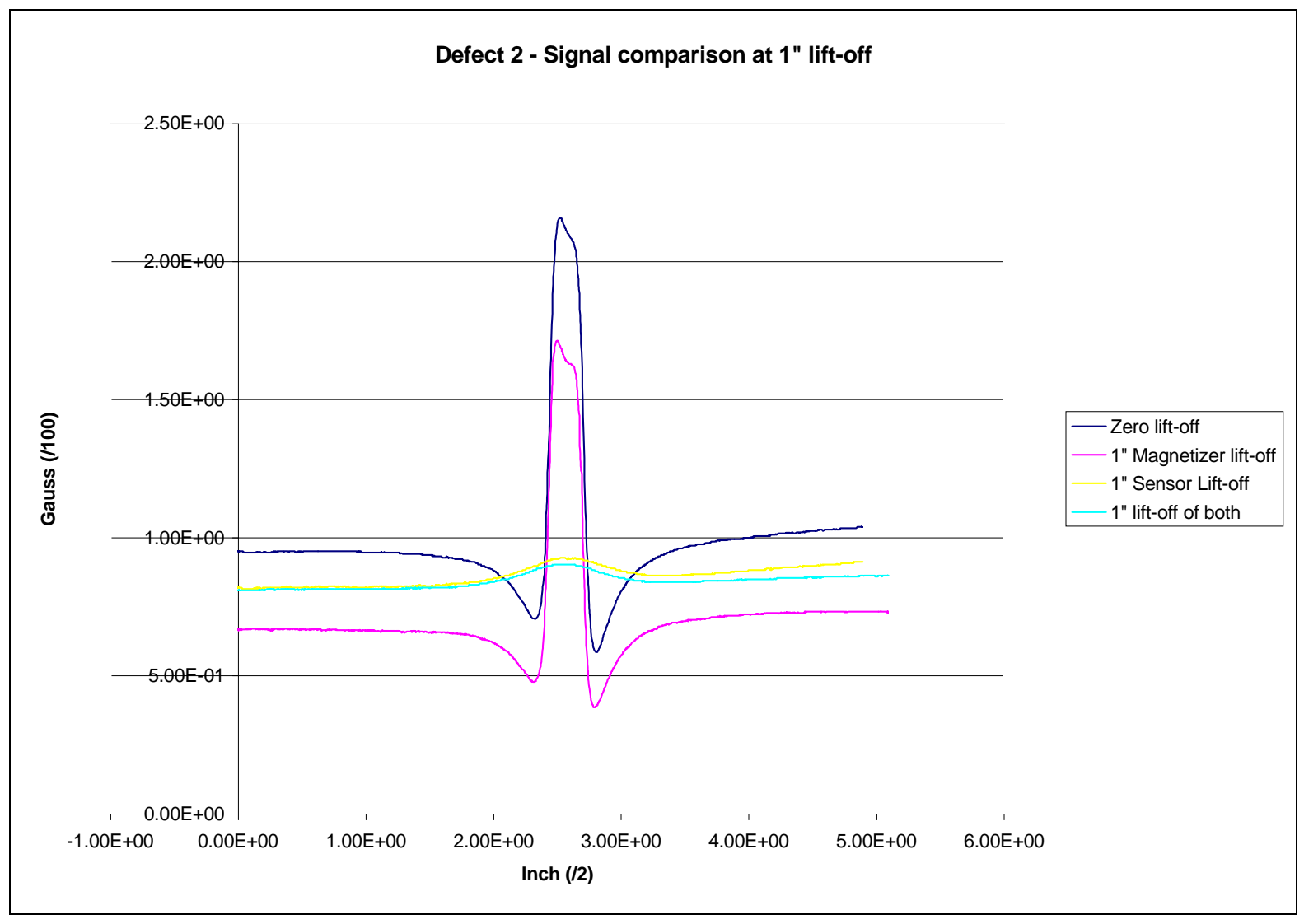


Appendix 16 - Defect 3 - Magnetic flux leakage signal comparison graphs
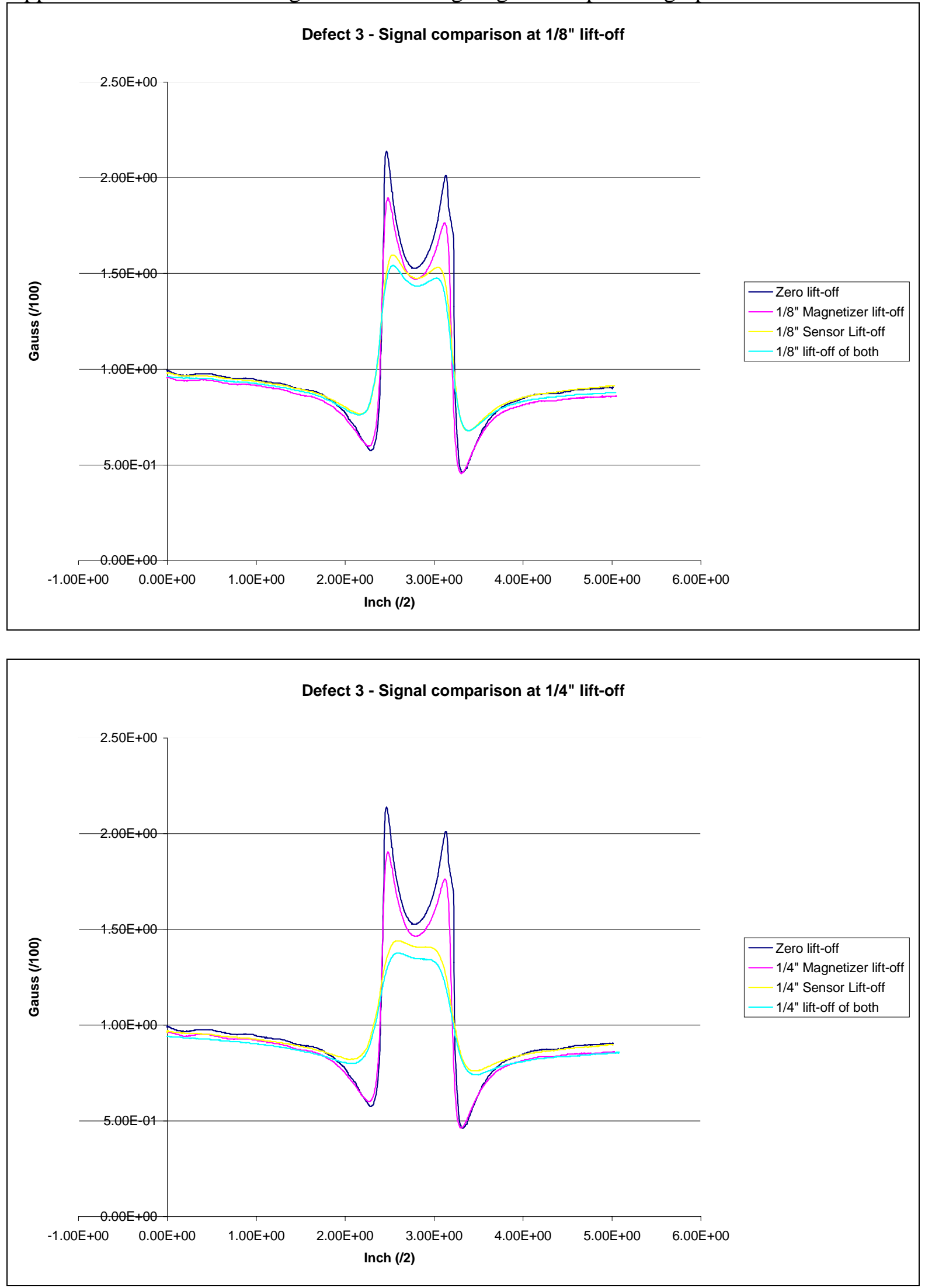

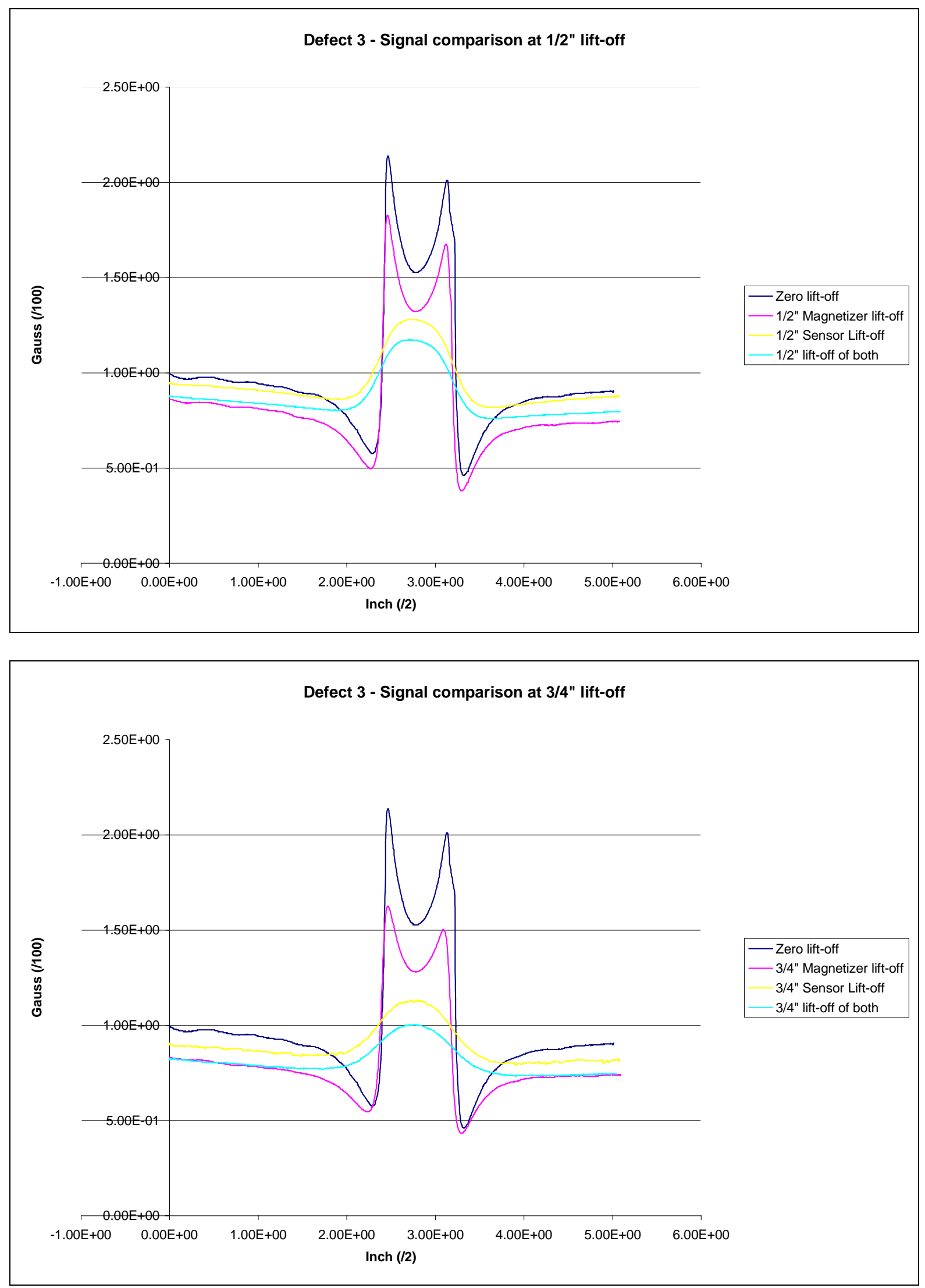


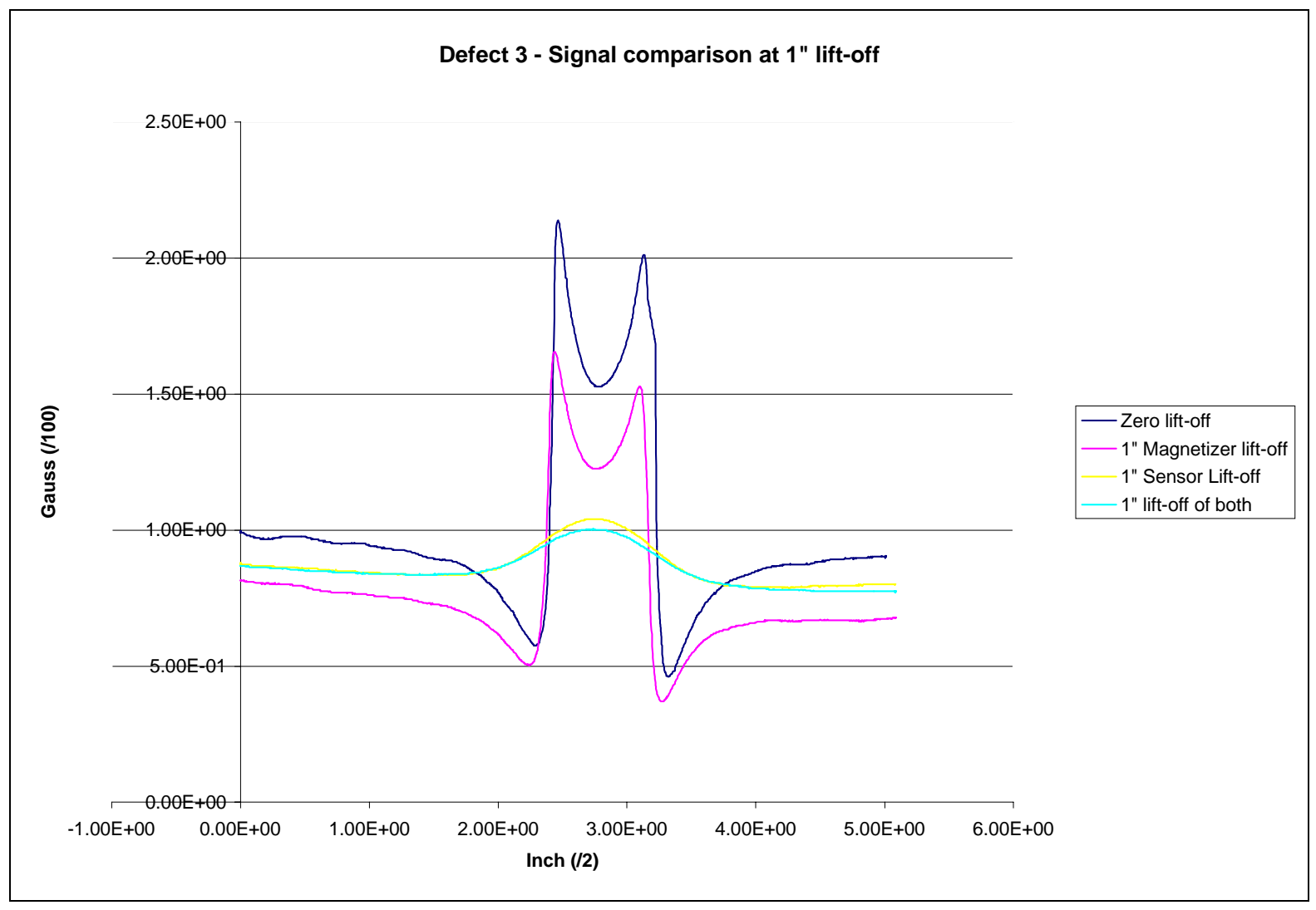


Appendix 17 - Defect 4 - Magnetic flux leakage signal comparison graphs
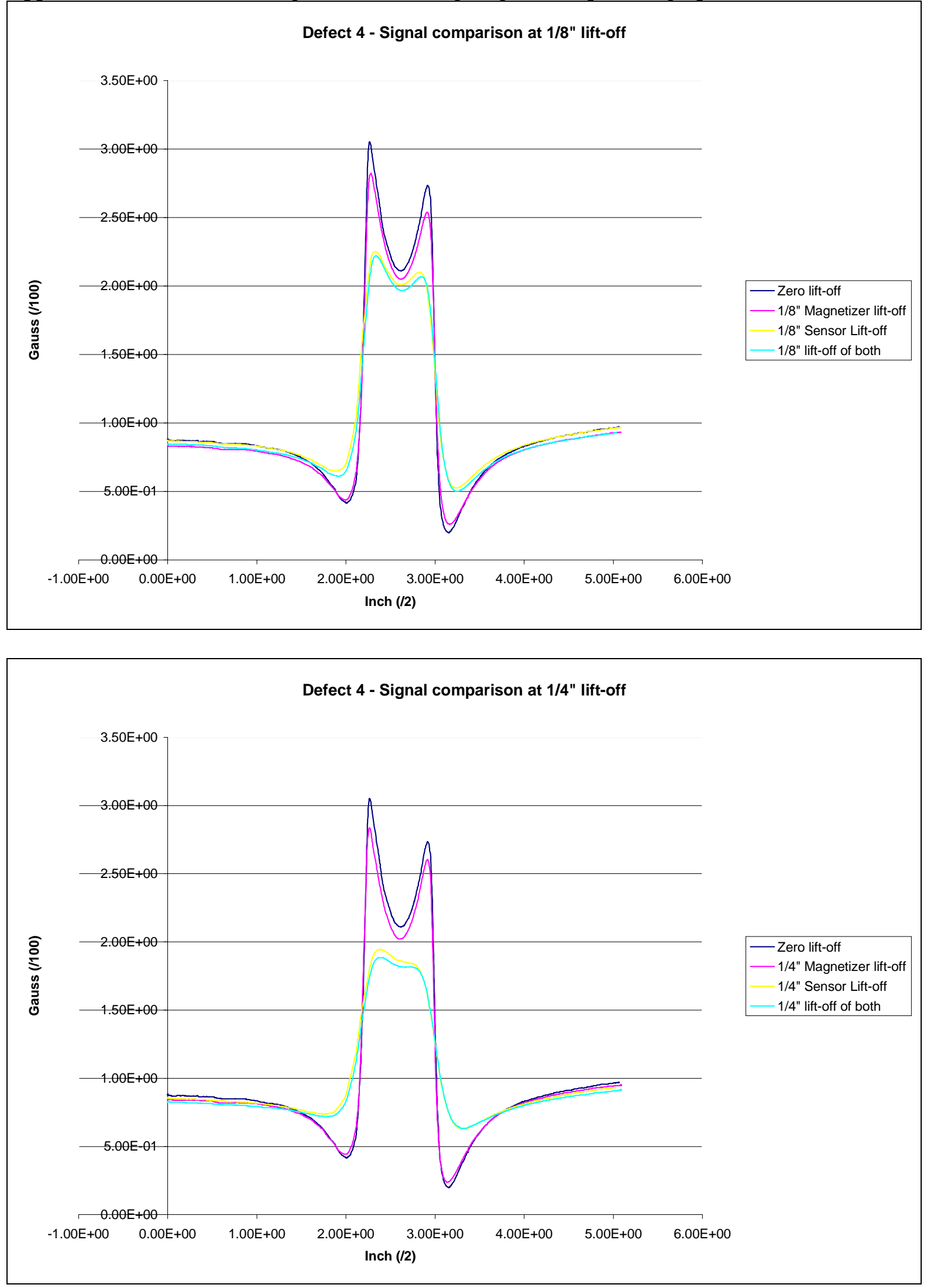

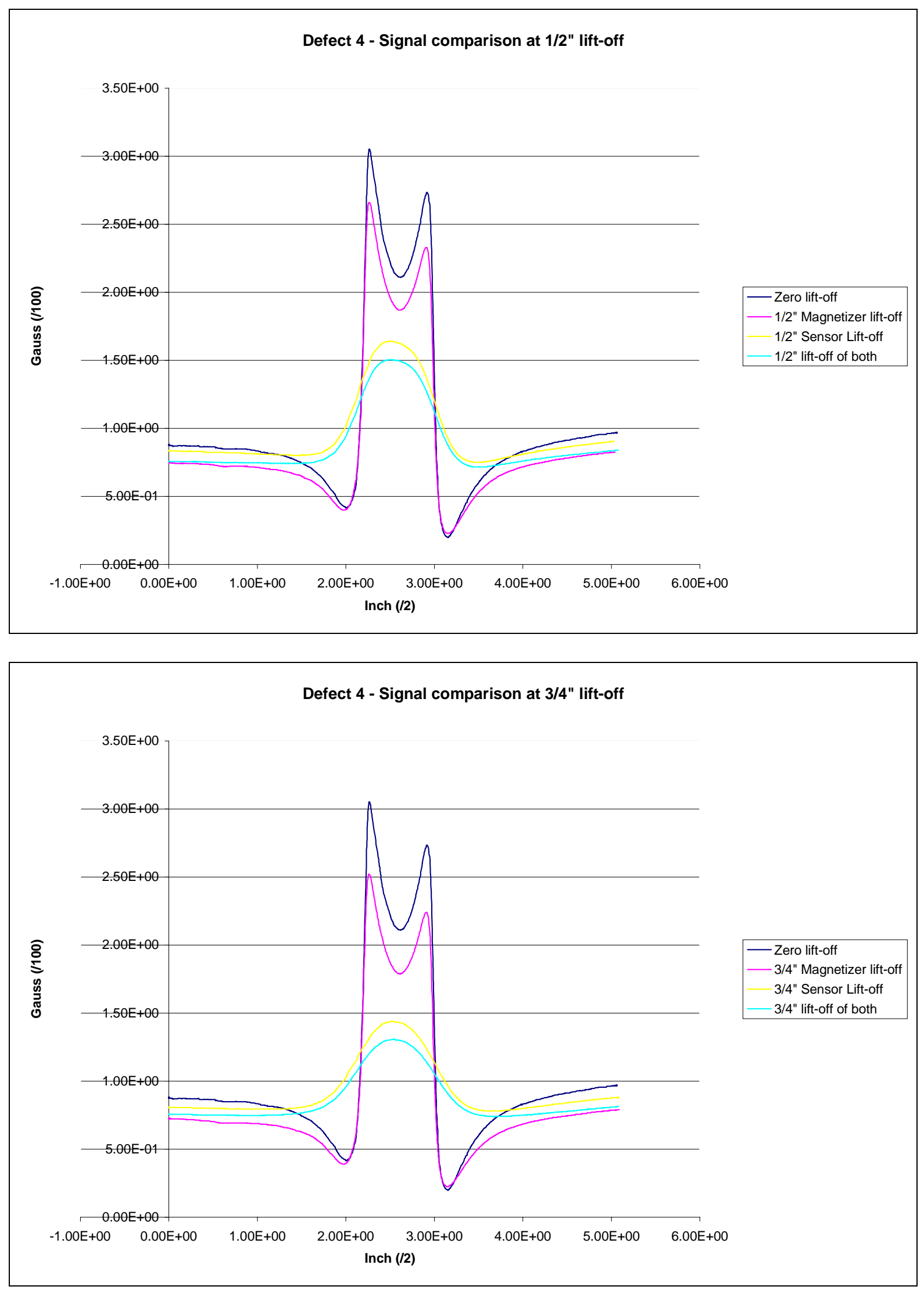


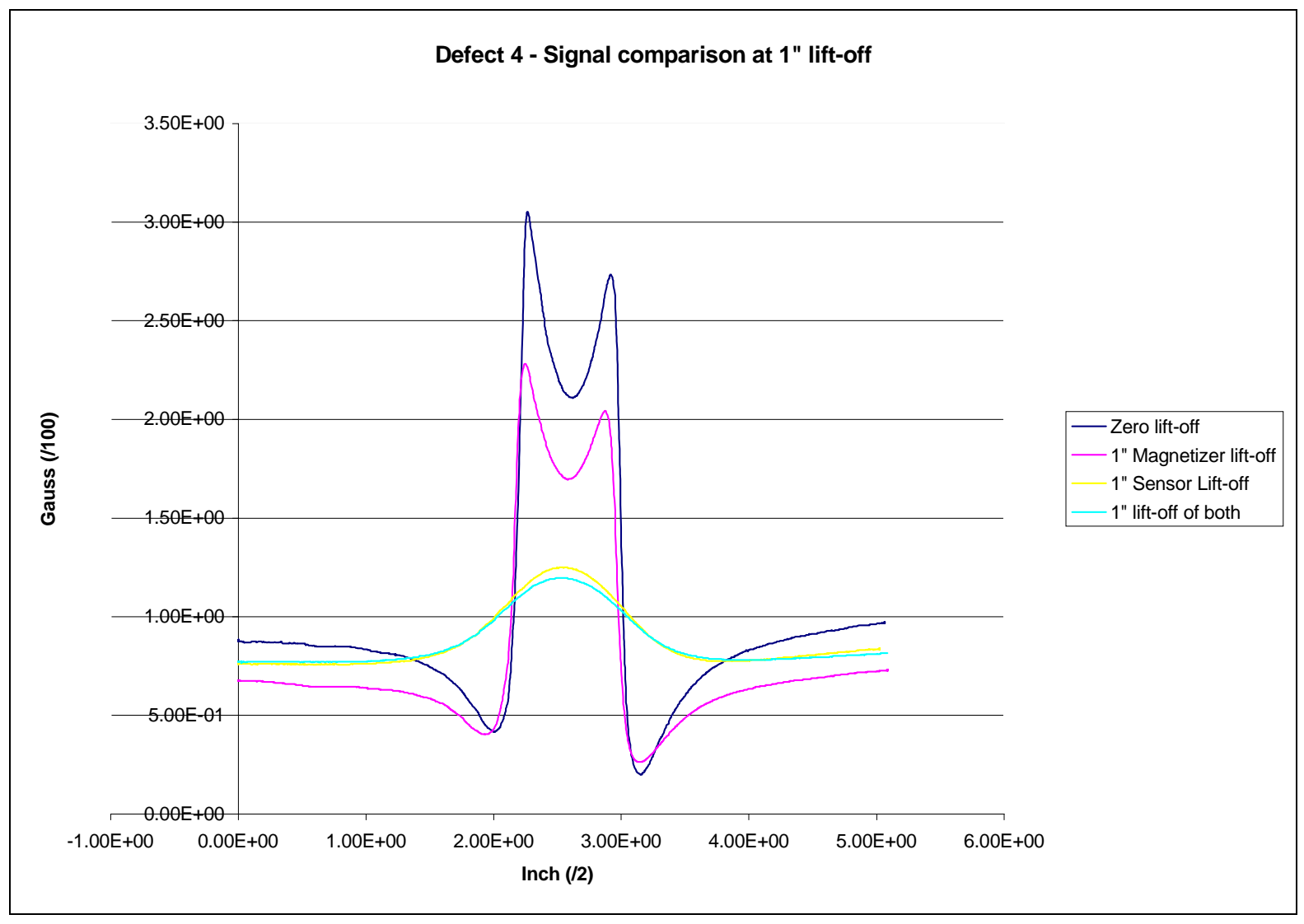


Appendix 18 - Defect 5 - Magnetic flux leakage signal comparison graphs
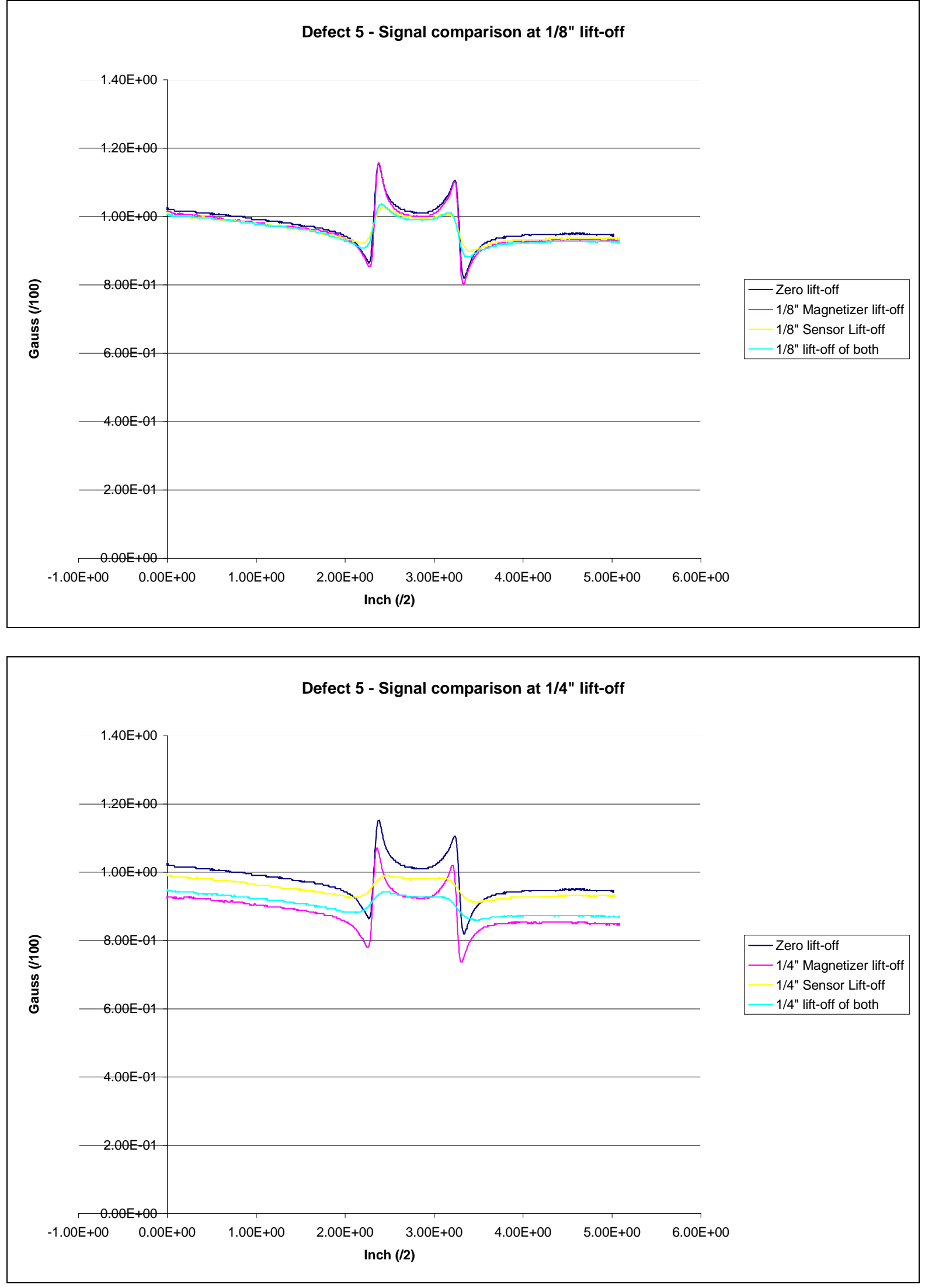

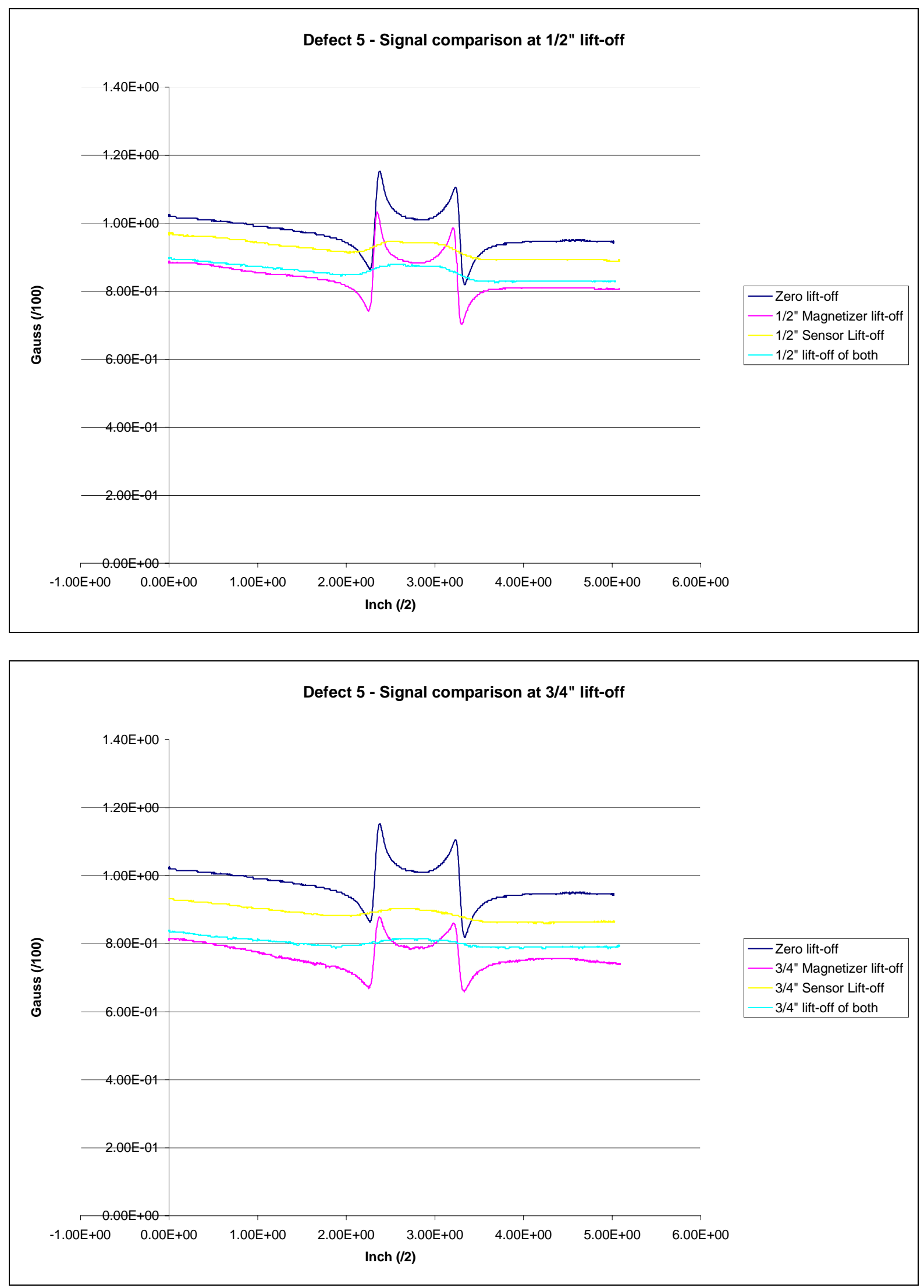


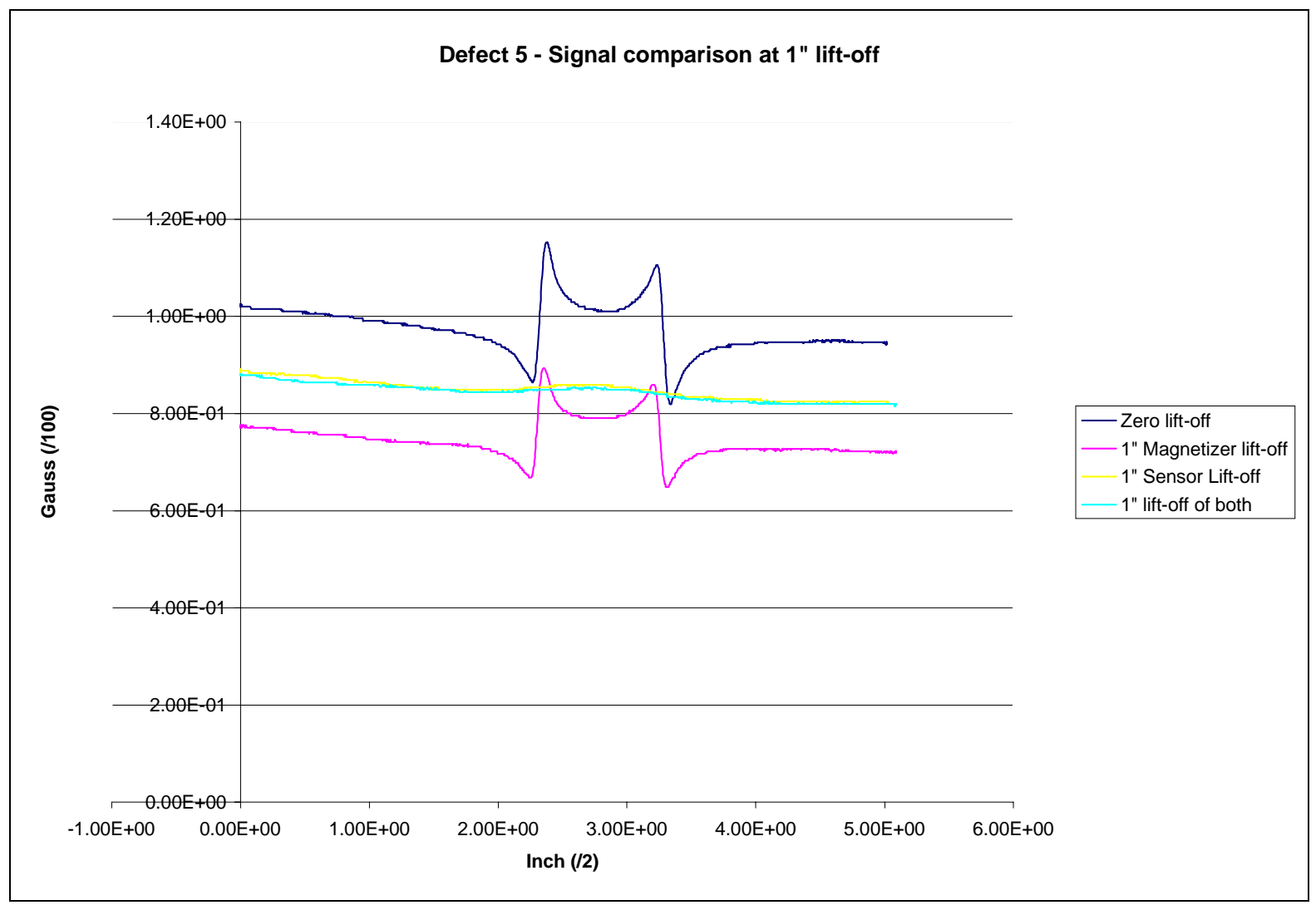


Appendix 19 - Defect 6 - Magnetic flux leakage signal comparison graphs
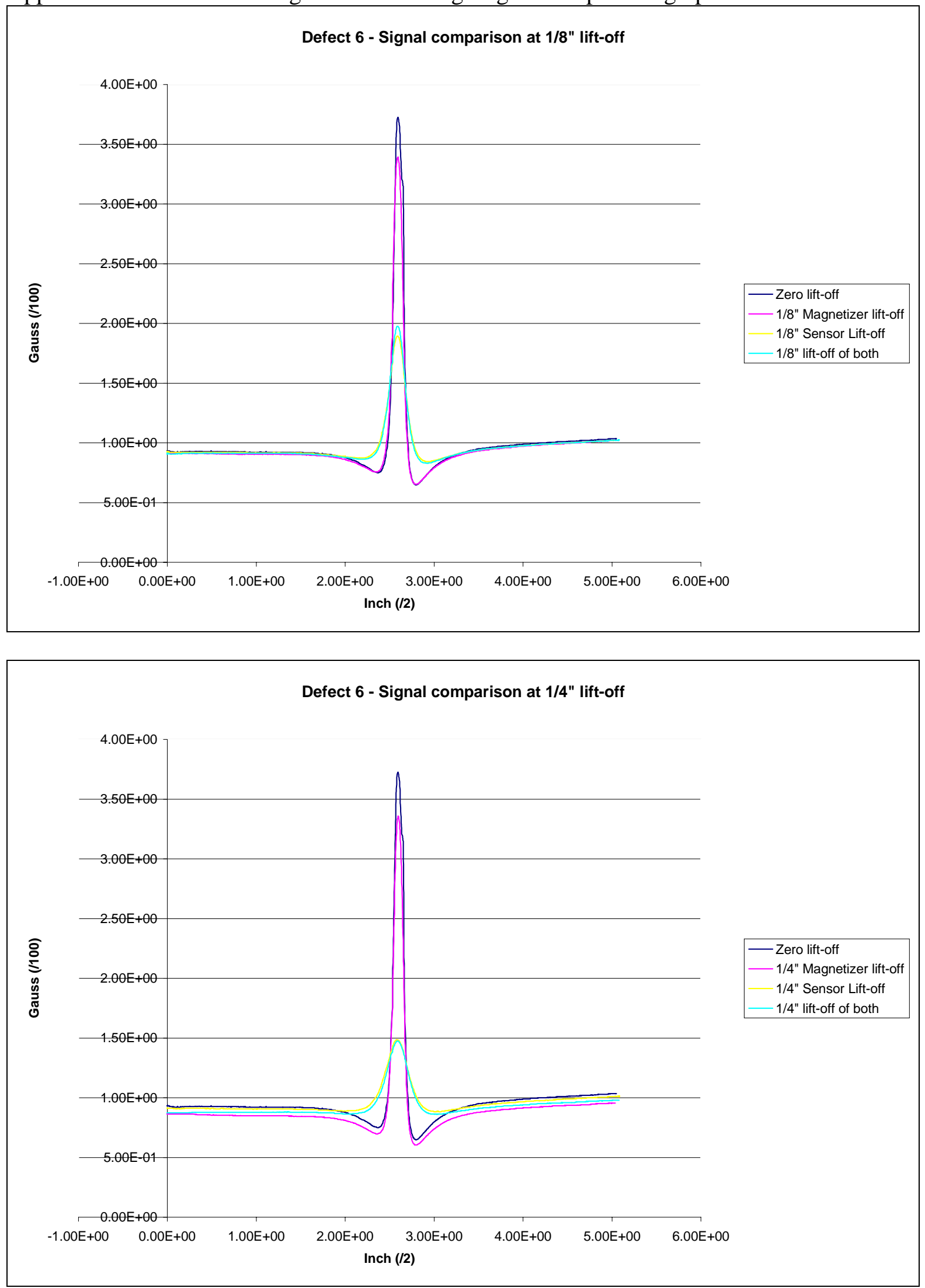

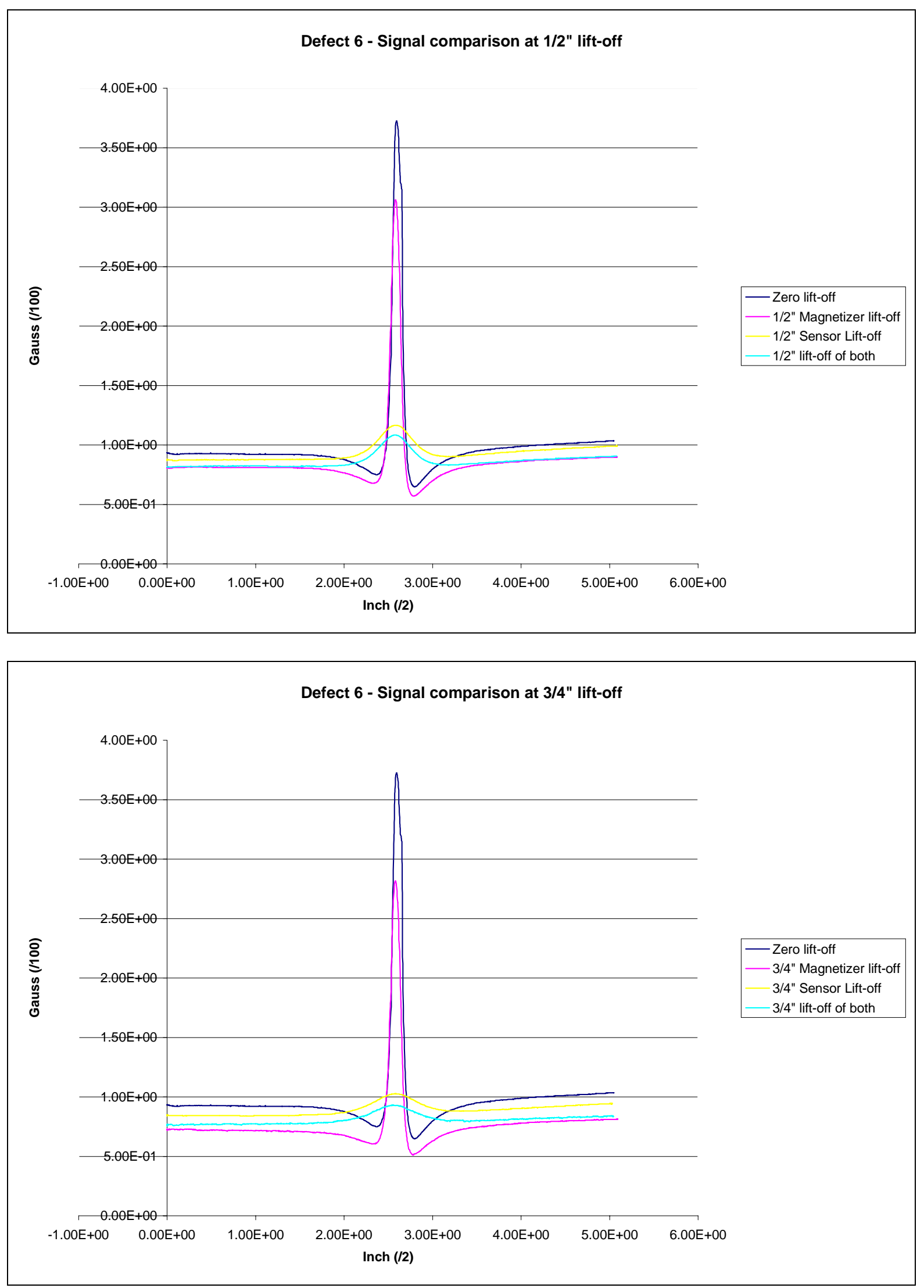


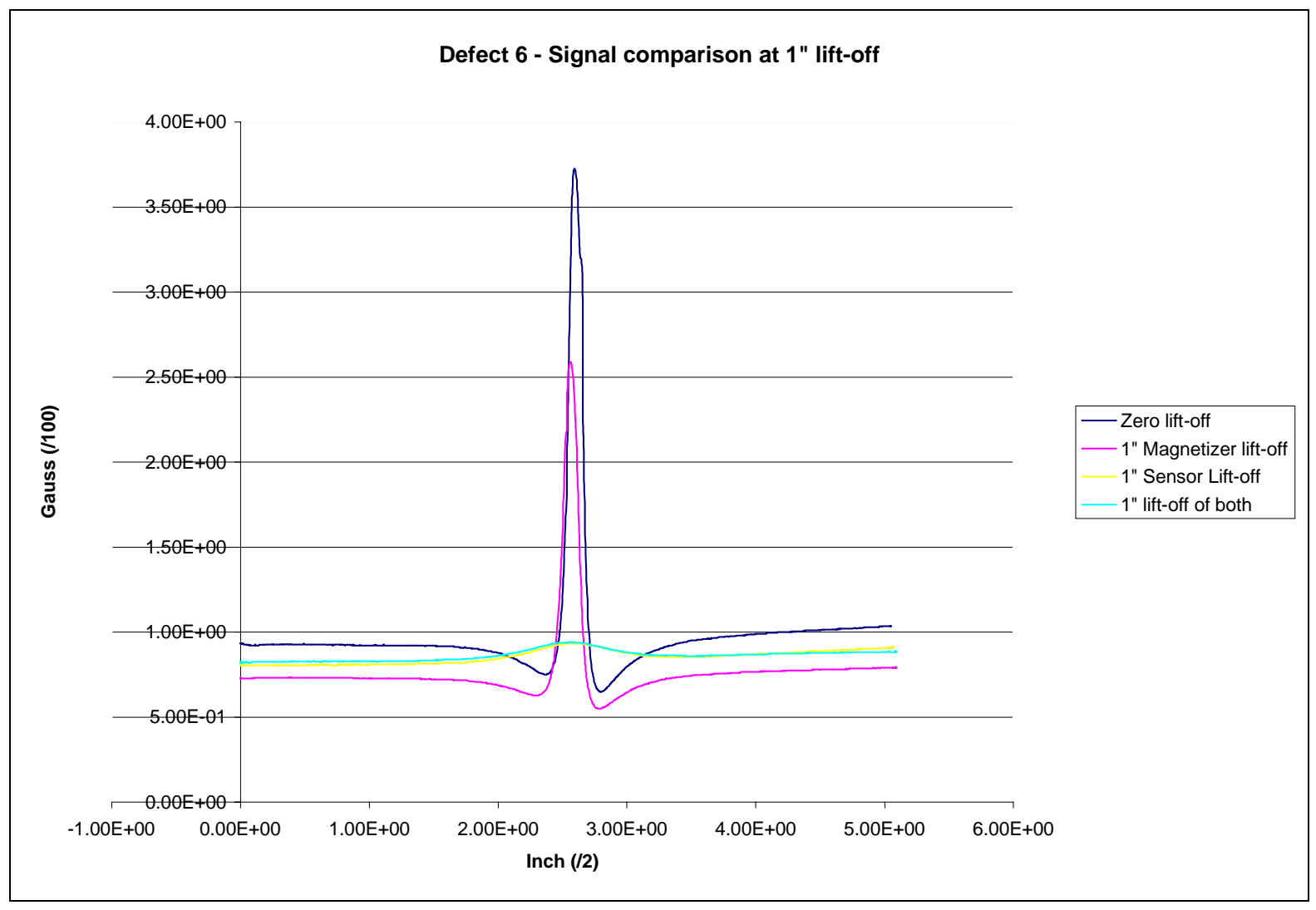


Appendix 20 - Peak magnetic flux leakage amplitude vs. lift-off of sensor, magnetizer, and both
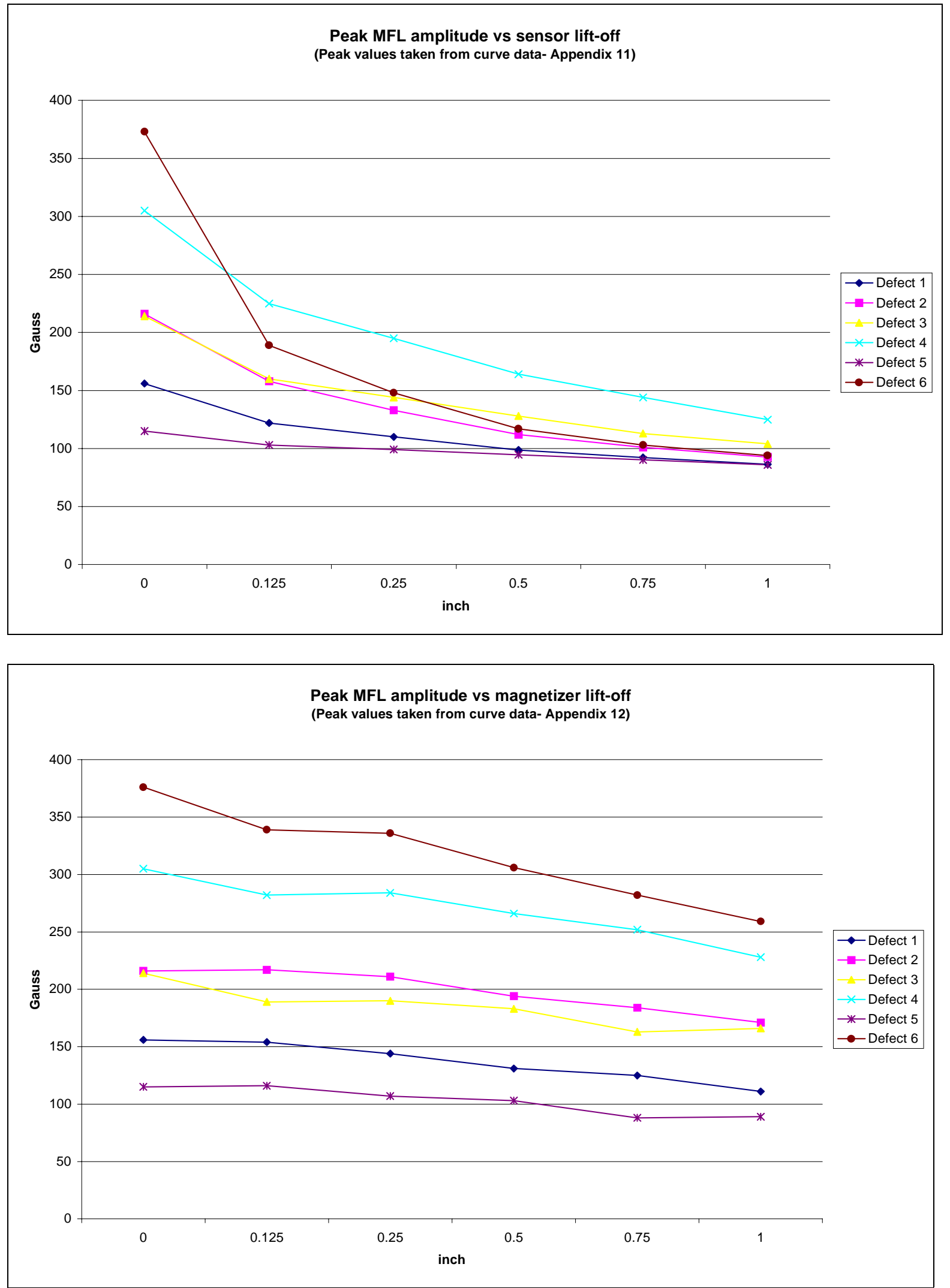


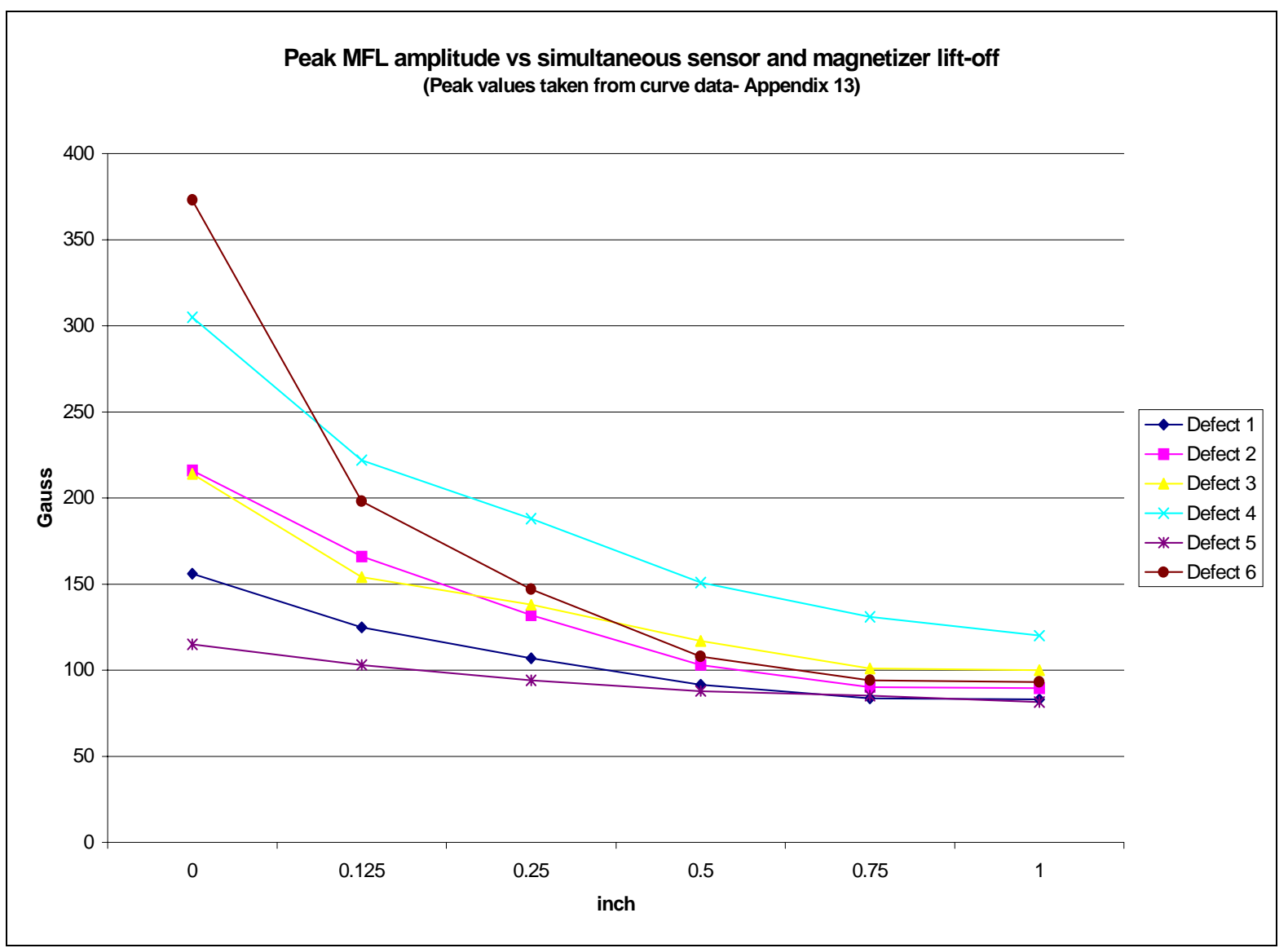


Appendix 21 - Percent reduction in magnetic flux leakage amplitude vs. increment of lift-off of sensor, magnetizer, and both
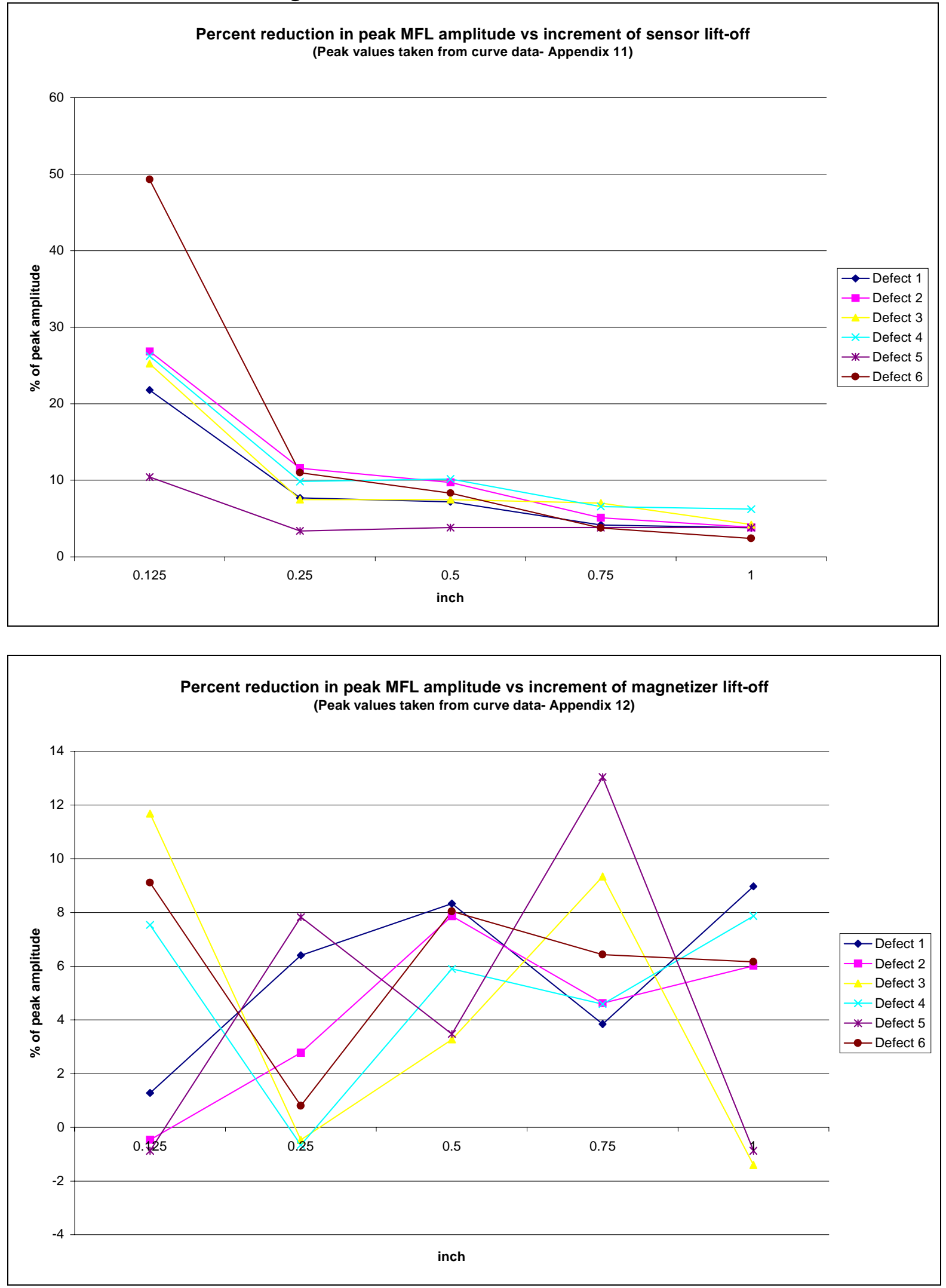


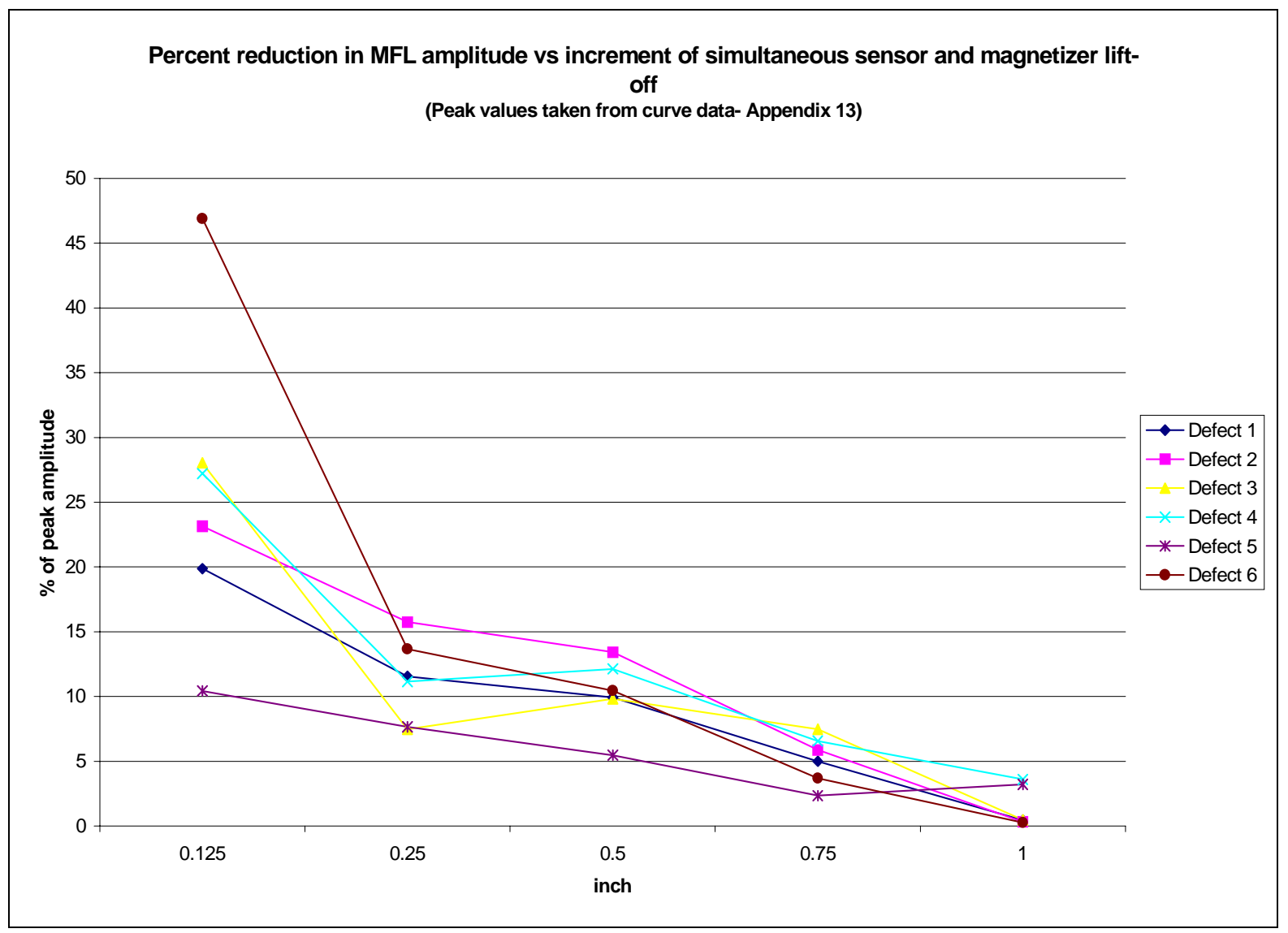


Appendix 22 - Baseline magnetic flux leakage amplitude vs. lift-off of sensor, magnetizer, and both
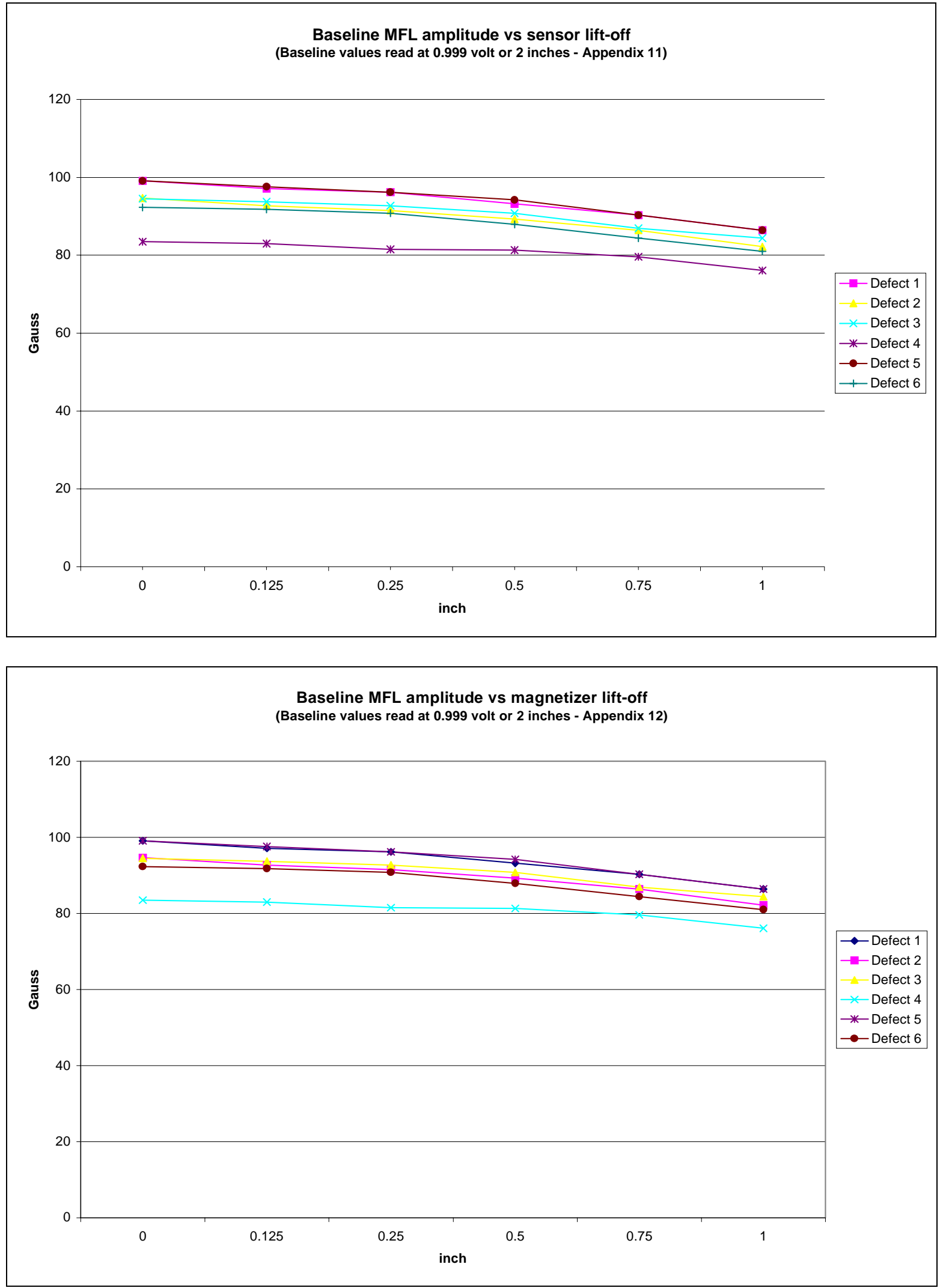


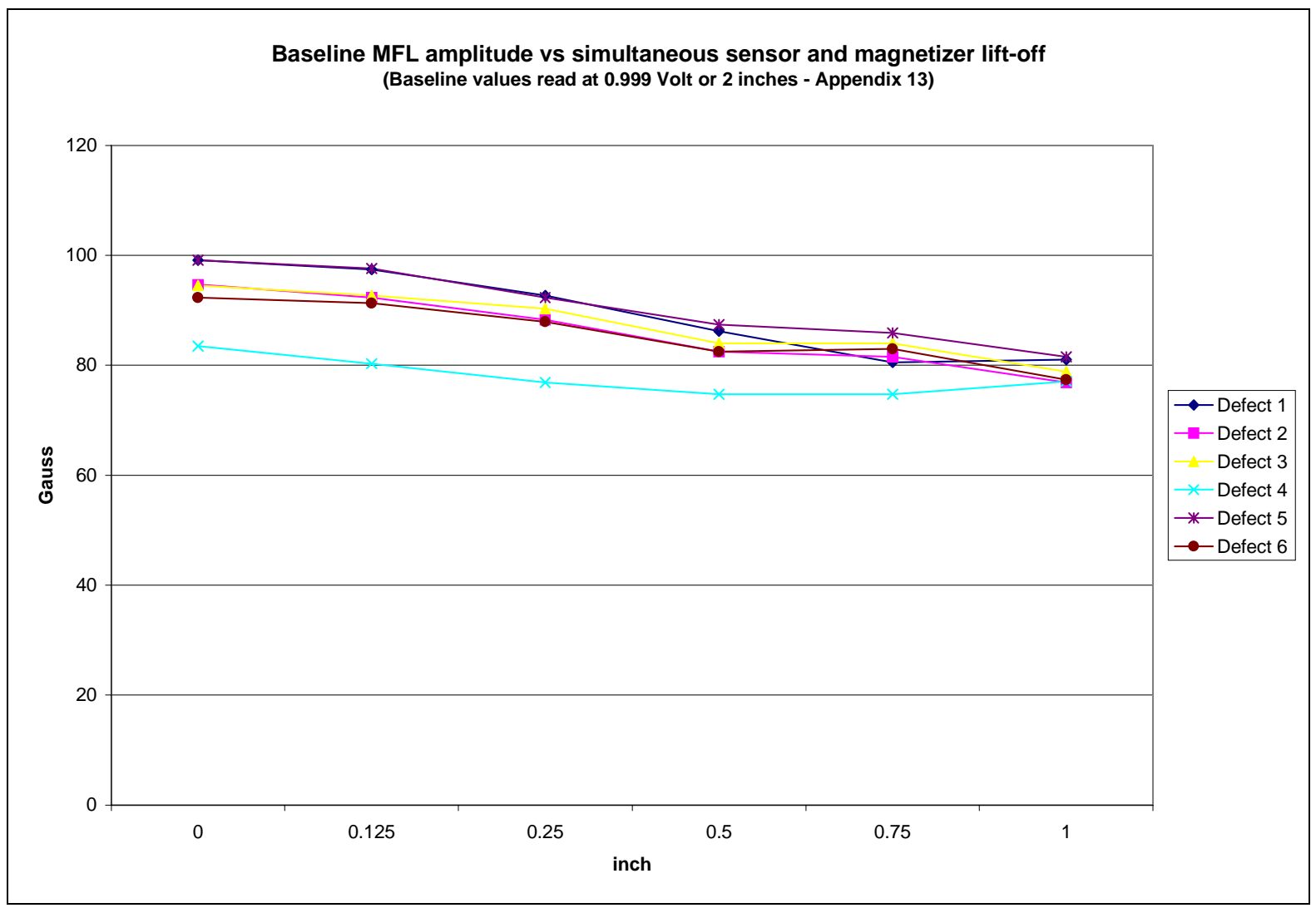


Appendix 23 - Percent reduction in baseline magnetic flux leakage amplitude vs. increment of lift-off of sensor, magnetizer, and both
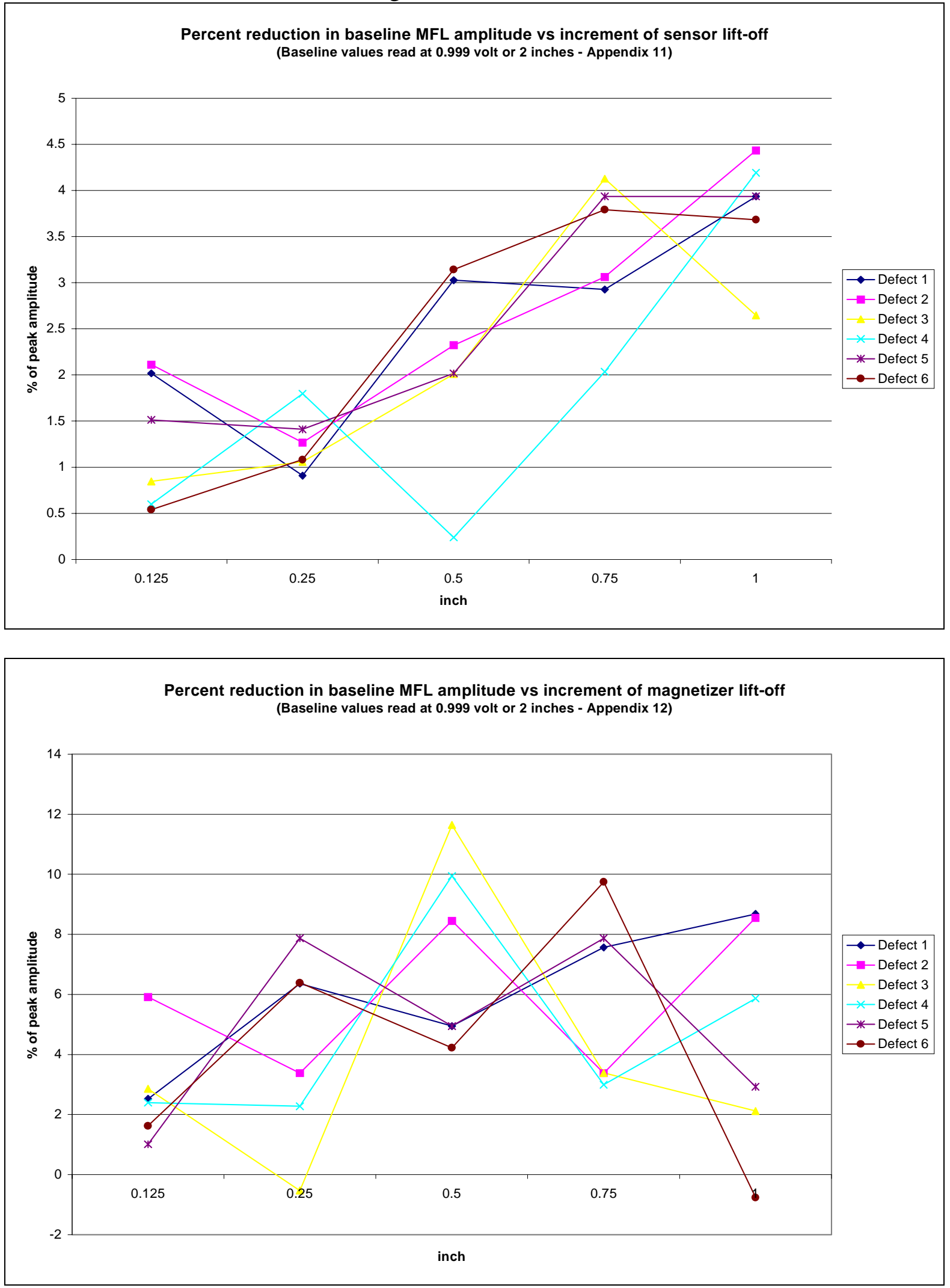


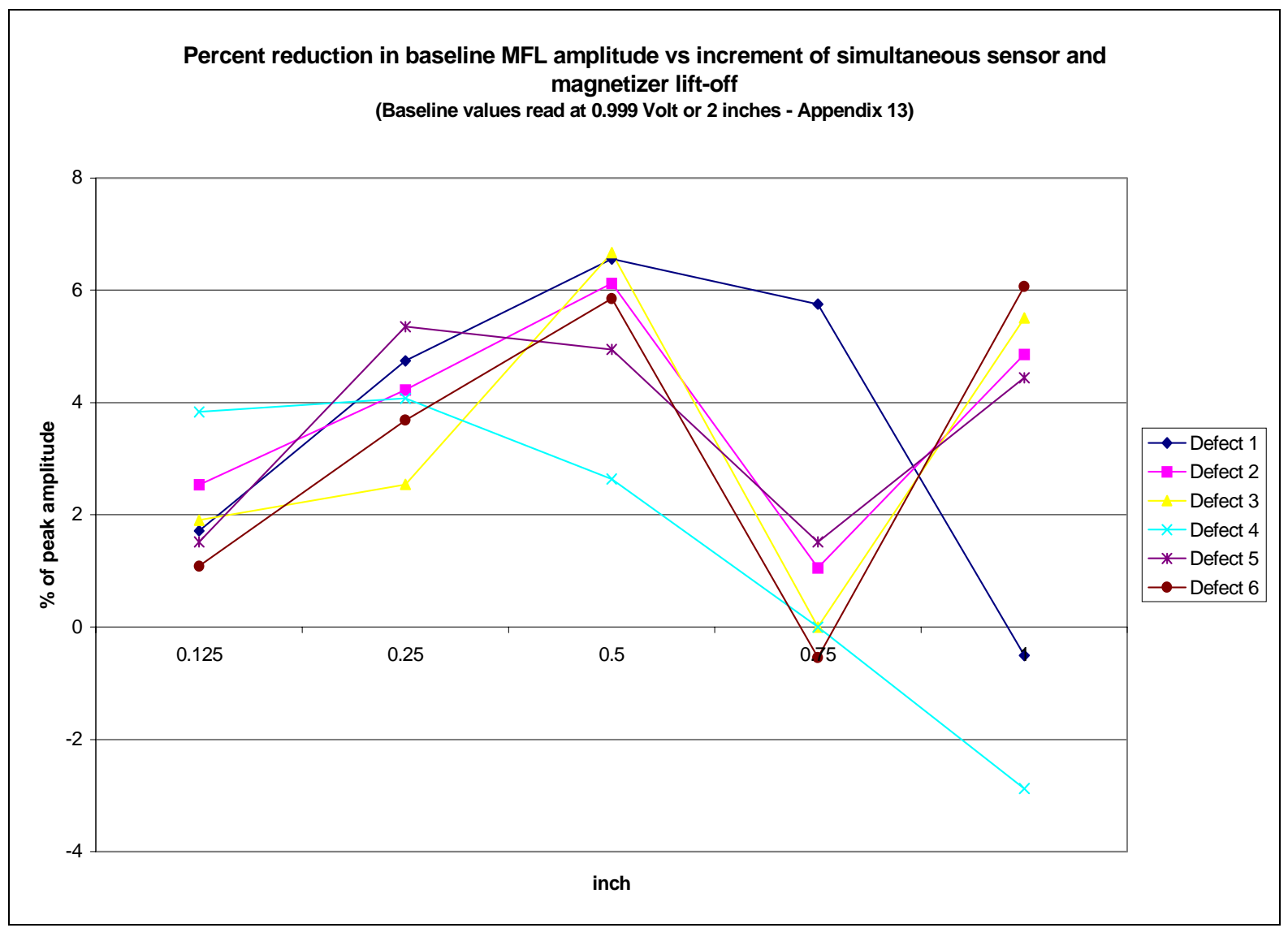




\section{VITA}

Francisco L. Valentine was born on Clark Air Force Base, Philippines on May 24, 1970. He graduated from Dupont High School in Belle, West Virginia in 1988. He received a Bachelor of Science Degree in Mechanical Engineering from West Virginia University in May 1992. He is currently a candidate for a Masters of Science Degree in Mechanical Engineering form West Virginia University.

Francisco worked as a Field Engineering Technician for three years and an Engineer for two years while employed with Columbia Gas Transmission Corporation from October of 1994 to February of 2000. Work at Columbia Gas Transmission Corporation dealt with the operation and maintenance of natural gas pipelines. He is currently employed as a Project Engineer with HEC-Energy Applications of Columbia, Maryland. HEC-Energy Applications is a heating ventilation and air-conditioning consulting firm. 